\title{
Galaxy alignments: Theory, modelling and simulations
}

\author{
Alina Kiessling ${ }^{1}$, Marcello Cacciato ${ }^{2}$, Benjamin Joachimi ${ }^{3}$, Donnacha Kirk ${ }^{3}$, Thomas D. \\ Kitching $^{4}$, Adrienne Leonard ${ }^{3}$, Rachel Mandelbaum ${ }^{5}$, Björn Malte Schäfer ${ }^{6}$, Cristóbal Sifón ${ }^{2}$, \\ Michael L. Brown ${ }^{7}$, Anais Rassat ${ }^{8}$ \\ Alina.A.Kiessling@jpl.nasa.gov
}

\begin{abstract}
The shapes of galaxies are not randomly oriented on the sky. During the galaxy formation and evolution process, environment has a strong influence, as tidal gravitational fields in the large-scale structure tend to align nearby galaxies. Additionally, events such as galaxy mergers affect the relative alignments of both the shapes and angular momenta of galaxies throughout their history. These "intrinsic galaxy alignments" are known to exist, but are still poorly understood. This review will offer a pedagogical introduction to the current theories that describe intrinsic galaxy alignments, including the apparent difference in intrinsic alignment between early- and late-type galaxies and the latest efforts to model them analytically. It will then describe the ongoing efforts to simulate intrinsic alignments using both $N$-body and hydrodynamic simulations. Due to the relative youth of this field, there is still much to be done to understand intrinsic galaxy alignments and this review summarises the current state of the field, providing a solid basis for future work.
\end{abstract}

Subject headings: galaxies: evolution; galaxies: haloes; galaxies: interactions; large-scale structure of Universe; gravitational lensing: weak

\section{Contents}

1 Introduction 3

2 Observables 4

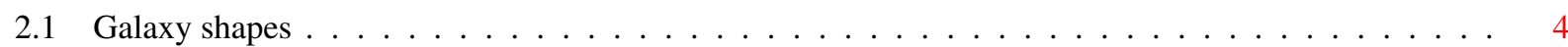

2.2 Alignment angles and types . . . . . . . . . . . . . . . . . . . 5

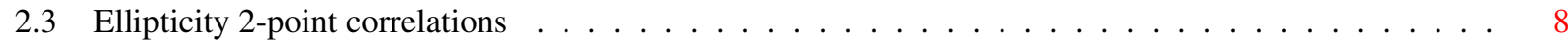

3 Theory and modelling 10

3.1 Large-scale alignments . . . . . . . . . . . . . . . . . . . . . . . . . 10

3.1 .1 Tidal interactions of haloes . . . . . . . . . . . . . . . . . . . . 10

\footnotetext{
${ }^{1}$ Jet Propulsion Laboratory, California Institute of Technology, 4800 Oak Grove Drive, Pasadena, CA, 91109, USA

${ }^{2}$ Leiden Observatory, Leiden University, PO Box 9513, 2300 RA, Leiden, the Netherlands

${ }^{3}$ Department of Physics and Astronomy, University College London, Gower Street, London WC1E 6BT, UK

${ }^{4}$ Mullard Space Science Laboratory, University College London, Holmbury St Mary, Dorking, Surrey RH5 6NT, UK

${ }^{5} \mathrm{McWilliams}$ Center for Cosmology, Department of Physics, Carnegie Mellon University, Pittsburgh, PA 15213, USA

${ }^{6}$ Astronomisches Recheninstitut, Zentrum für Astronomie der Universität Heidelberg, Philosophenweg 12, 69120 Heidelberg, Germany

${ }^{7}$ Jodrell Bank Centre for Astrophysics, School of Physics and Astronomy, University of Manchester, Oxford Road, Manchester M13 9PL, UK

${ }^{8}$ Laboratoire d'astrophysique (LASTRO), Ecole Polytechnique Fédérale de Lausanne (EPFL), Observatoire de Sauverny, CH-1290 Versoix, Switzerland
} 
3.1.2 Linear alignment model for elliptical galaxies . . . . . . . . . . . . . . . . . . 11

3.1 .3 Quadratic alignment model for spiral galaxies . . . . . . . . . . . . . . . . . . 13

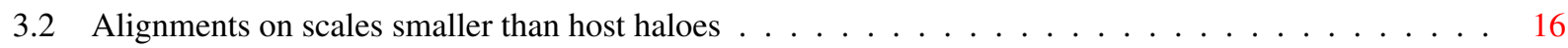

3.3 Transition to intermediate scales $\ldots \ldots \ldots \ldots \ldots \ldots \ldots \ldots \ldots$

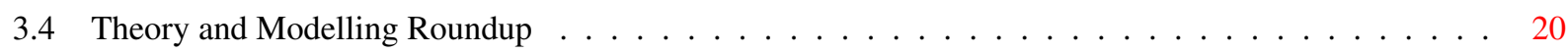

$4 \quad N$-Body simulations $\quad 20$

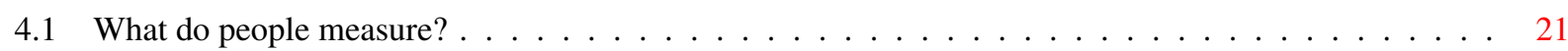

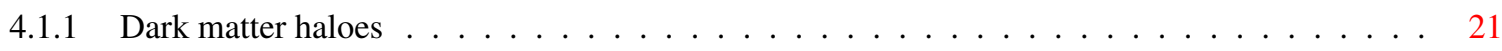

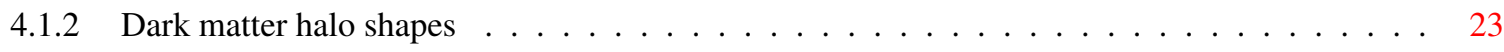

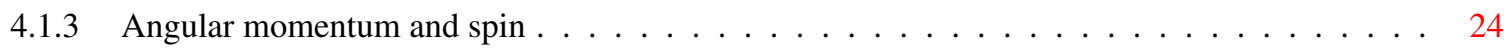

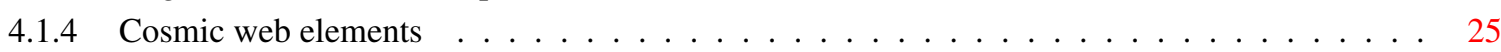

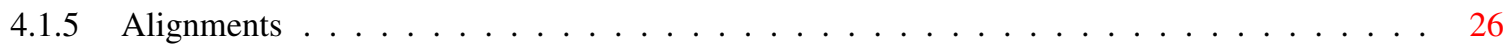

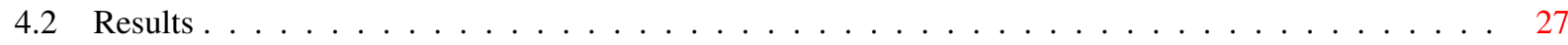

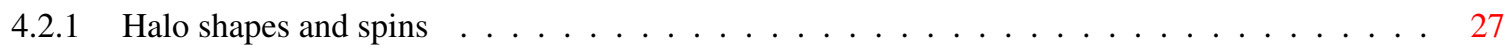

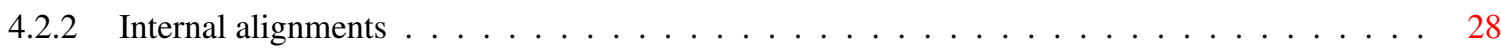

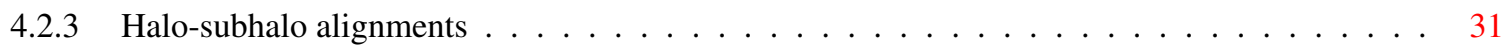

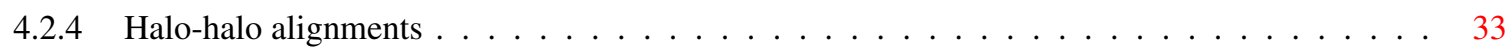

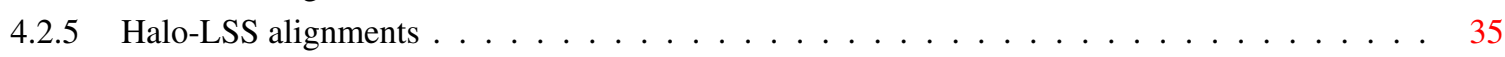

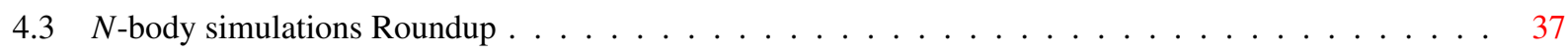

5 Hydrodynamic simulations 38

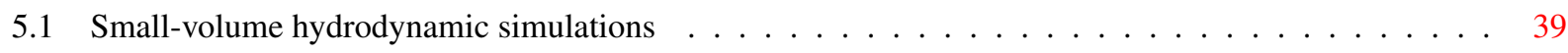

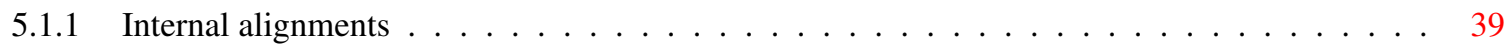

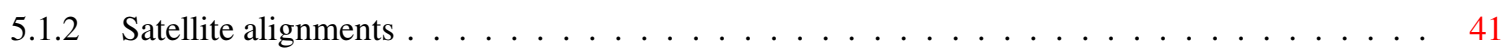

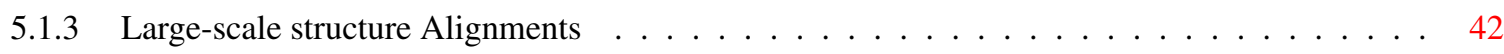

5.2 Cosmological-volume hydrodynamic simulations $\ldots \ldots \ldots \ldots \ldots \ldots \ldots \ldots \ldots \ldots$

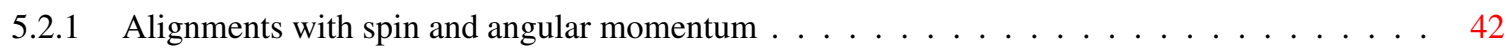

5.2 .2 Alignments with shape and position . . . . . . . . . . . . . . . . 43

5.3 Hydrodynamic simulations Roundup . . . . . . . . . . . . . . . . . . . . 47

6 Semi-analytic modelling $\quad 47$

7 Roadmap/wish list $\quad 49$

7.1 Simulations with sufficient resolution and numbers of haloes $\ldots \ldots \ldots \ldots \ldots$

7.2 A mapping between hydrodynamic and dark matter-only simulations $\ldots \ldots \ldots \ldots \ldots$

7.3 Accurate galaxy properties in hydrodynamic simulations $\ldots \ldots \ldots \ldots \ldots \ldots$

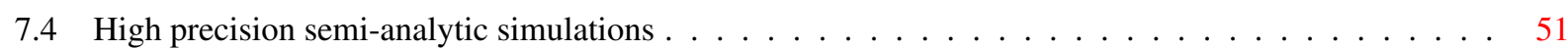

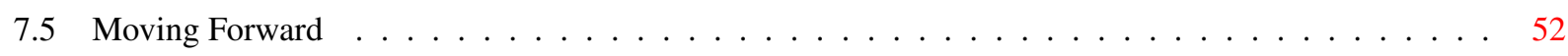

8 Concluding Remarks $\quad 52$ 


\section{Introduction}

The Universe is filled with galaxies, enormous collections of gas, dust and billions of stars all held together by gravity. Galaxies reside in the centre of dark matter haloes that contain up to $95 \%$ of the mass of the galaxy (see e.g. Coupon et al. 2015, and references therein). The current picture of structure formation is hierarchical with galaxies forming through anisotropic collapse of localised overdensities. Larger dark matter haloes are then formed by steady mass accretion of surrounding dark matter and through mergers with other haloes (including the galaxies they contain). These larger dark matter haloes contain many tens or hundreds of galaxies and are known as groups $\left(\sim 10^{13} \mathrm{M}_{\odot}\right)$ or clusters $\left(\gtrsim 10^{14} \mathrm{M}_{\odot}\right)$, which are the largest gravitationally bound structures in the Universe (see Dodelson 2003 for an introduction to structure formation).

Given that the Universe is homogeneous and isotropic on large scales, it was commonly assumed for some time that the observed galaxies would have random orientations if a large enough sample were considered. However, it is now known that local physical processes, in addition to initial conditions, have a strong influence on the alignment of galaxies with respect to their surrounding environment and that these alignments can be strongly anisotropic. These intrinsic alignments are not restricted simply to the orientation of the galaxy, but also to the rotational properties of galaxies. The physical processes acting on galaxies depend on many factors including whether the galaxy is early- or late-type (elliptical or spiral disc), red or blue (ellipticals with old stellar populations or spiral discs with active star formation). It is reasonable to assume that these factors may also have an impact on how the galaxy is aligned with surrounding structures (for a more complete introduction to galaxy formation and evolution, see Mo et al. 2010).

Observations are largely focused on investigating the alignments of galaxies, as opposed to dark matter haloes, since these are directly observed by telescopes ${ }^{1}$. However, cosmic structures grow much larger than galactic scales and this growth depends sensitively on the properties of dark matter and dark energy in an expanding Universe. Dark matter particles are only subject to gravity and can be approximated as experiencing no elastic collisions, which would give rise to pressure or viscous forces, in strong contrast to the sub-dominant baryonic component. Dark energy influences the growth of structures through the evolution of the background density with time and the relation between time and the scale factor of the Universe. Computationally, it is far easier to determine the alignments of dark matter haloes in $\mathrm{N}$-body dark matter simulations that include dark energy, since simulating baryons is still computationally expensive and it is unclear how to self-consistently model the dominant physical processes numerically over the large dynamic range involved in the problem at hand. There is a wealth of literature looking at the alignments of dark matter haloes with respect to substructures, other haloes and even the cosmic web. Even though the mass resolution of early simulations restricted the studies to cluster-sized haloes, more recent works have extended this to include groupand galaxy-sized haloes in addition to using semi-analytic models to add some of the key properties of galaxies and hydrodynamics to add baryonic processes to the simulations.

Understanding the alignments of galaxies, groups and clusters is important from a formation and evolution perspective. However, it is essential to galaxy surveys that include weak gravitational lensing as a key cosmological probe ${ }^{2}$ for a very different reason. Weak gravitational lensing exploits the correlations between the shapes of distant galaxy images, which have been distorted by the gravitational deflection of light by the intervening matter distribution (see Bartelmann \& Schneider 2001 for more information on weak gravitational lensing). This distortion or shear signal can be boosted by the intrinsic alignments of galaxies which mimic the gravitational shear, or diminished by intrinsic alignments of galaxies that are anti-correlated with the gravitational shear signal. If ignored, these can bias the inference on cosmology and represent the largest astrophysical systematic for upcoming weak gravitational lensing surveys (see Kirk et al. 2015). In order to mitigate the signal from these alignments without losing a significant amount of cosmological information, it is first necessary to formulate a reasonably accurate model of the alignments (see Kirk et al. 2015). Only then will it be possible to accurately quantify the effects and mitigate the bias that they induce .

This review is part of a topical volume on galaxy alignments and it provides an introduction to the theory, modelling

\footnotetext{
${ }^{1}$ However, there are methods to measure dark matter halo ellipticities through observations, e.g. galaxy-galaxy lensing (e.g. Natarajan \& Refregier 2000; Hoekstra et al. 2004; Mandelbaum et al. 2006a; Parker et al. 2007; van Uitert et al. 2012; Schrabback et al. 2015)

${ }^{2}$ e.g. the Kilo Degree Survey, KiDS: http://kids.strw.leidenuniv.nl; the Dark Energy Survey, DES: http://www.darkenergysurvey.org; the Hyper Suprime-Cam Survey, HSC: http://www.naoj.org/Projects/HSC; Euclid: http://www.euclid-ec.org and http://sci.esa.int/euclid; the Large Synoptic Survey Telescope, LSST: http://www.lsst.org/lsst; and the Wide Field InfraRed Suvery Telescope, WFIRST: http://wfirst.gsfc.nasa.gov
} 
and simulations of alignments of structures within the Universe. Also in this volume is a basic overview of the galaxy alignments (Joachimi et al. 2015) and a more technical review on observational results, the impact on cosmology and mitigation techniques (Kirk et al. 2015).

This review first defines alignment observables in Section 2, to give context to the alignments being modelled or simulated throughout. The large-, small- and intermediate-scale theories and models for alignments are then reviewed in Section 3. Section 4 summarises the results of alignments in $N$-body simulations, while Section 5 reviews results from hydrodynamic simulations. Section 6 introduces semi-analytic modelling and this is followed by a roadmap or wish list for future investigations into galaxy intrinsic alignments, Section 7, and final remarks in Section 8.

\section{Observables}

In this section, the observable quantities that are measured in real data and predicted theoretically are defined. These include galaxy shapes (Section 2.1), the relative angles of interest between the astronomical structures (Section 2.2), and 2-point correlation functions (Section 2.3).

\subsection{Galaxy shapes}

When observing the sky, it is not possible to observe the full three-dimensional shapes of galaxies. The shapes measured are inherently two-dimensional projections of the three-dimensional shape. While galaxies and dark matter haloes do not in general have elliptical isophotes or isodensity contours, it is common to describe their shapes in terms of the effective ellipticity at some radius (or averaged over a range of radii, as in weak lensing; see Kirk et al. 2015). The observed ellipticity, $\epsilon$, can be split into two components

$$
\epsilon \simeq \epsilon^{\mathrm{s}}+\gamma,
$$

where $\epsilon^{\mathrm{s}}$ is the intrinsic ellipticity that corresponds to the true shape of the light distribution, and $\gamma$ is the gravitational shear ${ }^{3}$, a distortion to the galaxy image produced by intervening matter along the line of sight to the galaxy in question (see Joachimi et al. 2015 for a discussion of the approximation in this equation). There is a fundamental difference between $\epsilon^{\mathrm{S}}$ and $\gamma$ in that $\epsilon^{\mathrm{S}}$ describes the deviation of the true shape of the galaxy itself from circularity, while $\gamma$ is a distortion to the observed galaxy shape, and will depend on the matter distribution through each line of sight.

Ellipticities are tensorial quantities that have, in complex notation, two components (note that Equation 1 is also an equation between complex numbers). It is standard to define a fixed coordinate frame on the sky, with a position angle, $\varphi$, defining the angle of the semi-major axis of the ellipse from one axis of that coordinate frame. If the total magnitude of the ellipticity is $|\epsilon|$ as defined in terms of the semi-minor to semi-major axis ratio $q$ (see, e.g., Bernstein \& Jarvis 2002 for many common ellipticity definitions), then the two components of ellipticity in this coordinate system ${ }^{4}$ can be defined as $\epsilon_{1}=|\epsilon| \cos 2 \varphi$ and $\epsilon_{2}=|\epsilon| \sin 2 \varphi$, with the complex ellipticity denoted $\epsilon=\epsilon_{1}+\mathrm{i} \epsilon_{2}$, or equivalently $\epsilon=|\epsilon| \exp (2 \mathrm{i} \varphi)$. The factor of 2 by which the phase angle is multiplied takes care of the internal spin-2 symmetry of the ellipticity field, which is mapped onto itself after a rotation of the coordinates by $\pi$.

The notion of spatial ellipticity correlations and their link to correlation functions is illustrated here with the following model: The ellipticity of every galaxy can be seen as being drawn randomly from some underlying distribution, with a non-vanishing correlation function describing the dependence of the random processes assigning ellipticities to two neighbouring galaxies. If $\epsilon$ and $\epsilon^{\prime}$ are the ellipticities of galaxies at positions $\alpha$ and $\alpha^{\prime}$ respectively, their ellipticities are drawn from a multivariate Gaussian distribution $p\left(\epsilon^{\prime}, \epsilon\right)$, which can be viewed as a conditional distribution $p\left(\epsilon^{\prime} \mid \epsilon\right)$, such that the outcome $\epsilon^{\prime}$ depends on the value of $\epsilon$. As a multivariate distribution $p\left(\epsilon, \epsilon^{\prime}\right)$,

$$
p\left(\epsilon, \epsilon^{\prime}\right)=\frac{1}{\sqrt{(2 \pi)^{4} \operatorname{det}\left(C_{\epsilon}\right)}} \exp \left[-\frac{1}{2}\left(\begin{array}{c}
\epsilon \\
\epsilon^{\prime}
\end{array}\right)^{+} C_{\epsilon}^{-1}\left(\begin{array}{c}
\epsilon \\
\epsilon^{\prime}
\end{array}\right)\right],
$$

\footnotetext{
${ }^{3}$ The term gravitational shear could be misleading here since the intrinsic ellipticity, $\epsilon^{\mathrm{S}}$, is also influenced by (tidal) gravitational effects as shown in this section. However, the term gravitational shear for the effects of gravitational lensing has been in use for a long time and it would be unwise to adopt a different convention here. Throughout, the term gravitational shear is synonymous with gravitational lensing.

${ }^{4}$ If a two-component ellipticity seems unfamiliar, it is worth considering that the standard geometric representation of an ellipse using an axis ratio and a position angle also requires two numbers.
} 

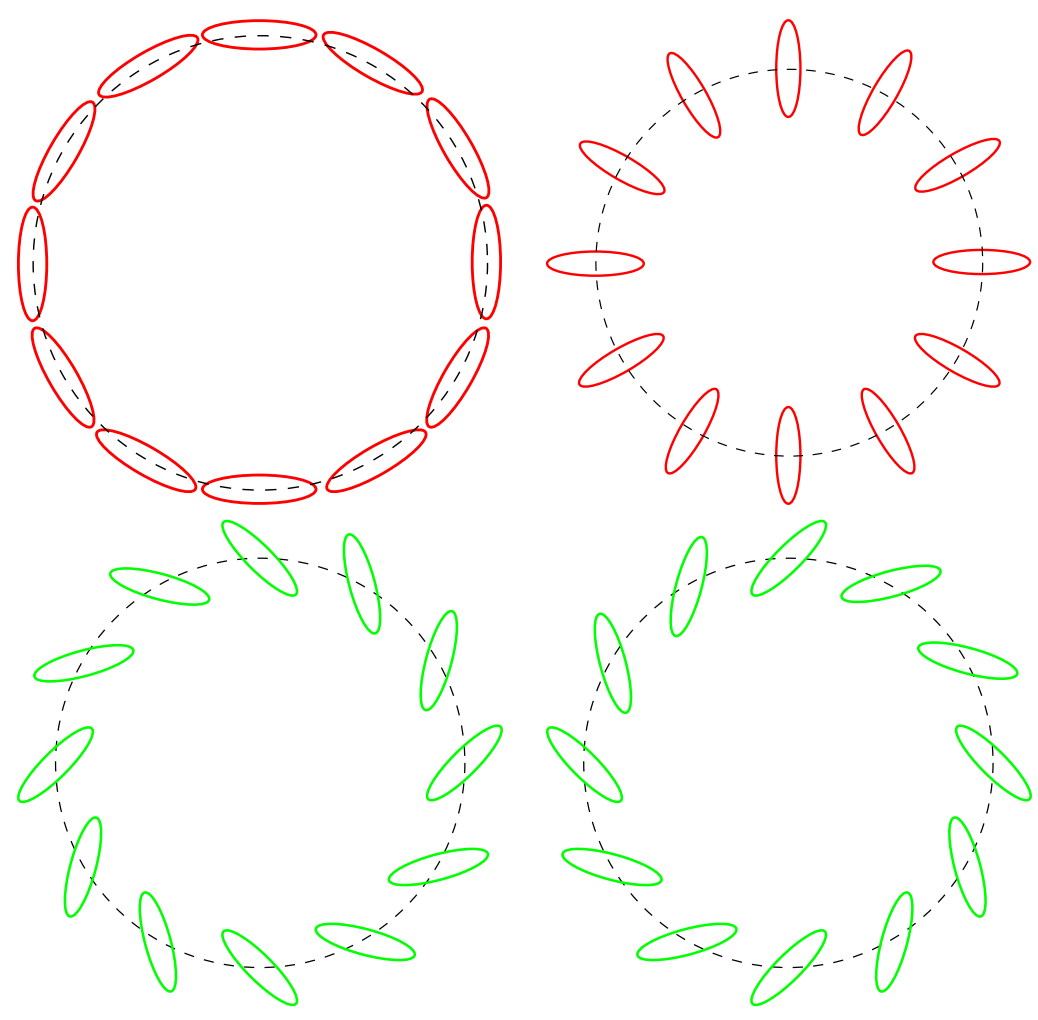

Fig. 1.-: Parity-even $E$-modes (top row) and the $B$-modes (bottom row) of the ellipticity field. Measured with respect to a radial line from the centre of the pattern, one observes $\epsilon_{+}>0 ; \epsilon_{\times}=0$ (top left), $\epsilon_{+}<0 ; \epsilon_{\times}=0$ (top right), $\epsilon_{+}=0 ; \epsilon_{\times}<0$ (bottom left), and $\epsilon_{+}=0 ; \epsilon_{\times}>0$ (bottom right), in the convention given by Equation (4).

this property is encoded in the covariance matrix $C_{\epsilon}$,

$$
C_{\epsilon}=\left(\begin{array}{cc}
\left\langle\epsilon \epsilon^{*}\right\rangle & \left\langle\epsilon \epsilon^{\prime *}\right\rangle \\
\left\langle\epsilon^{*} \epsilon^{\prime}\right\rangle & \left\langle\epsilon^{\prime} \epsilon^{\prime *}\right\rangle
\end{array}\right)
$$

Note that, since $\epsilon$ is a complex number, the covariance $C_{\epsilon}$ is Hermitian, with the asterisk denoting the complex conjugate and the plus sign in Equation (2) denoting the Hermitian conjugate. The two variances $\left\langle|\epsilon|^{2}\right\rangle$ and $\left\langle\left|\epsilon^{\prime}\right|^{2}\right\rangle$ are equal in homogeneous random fields, because the fluctuation properties are identical everywhere. The off-diagonal element is the correlation function $\xi_{\epsilon}=\left\langle\epsilon \epsilon^{\prime *}\right\rangle$, which is invariant under translation in homogeneous fields. It generally decreases with increasing distance due to the Cauchy-Schwarz inequality, $\xi_{\epsilon} \leq\left\langle|\epsilon|^{2}\right\rangle$, and describes how rapidly the fluctuating field loses memory of its value $\epsilon$ at $\alpha$ when increasing the distance to $\alpha^{\prime}$. If the random field is isotropic, the random field's correlation properties do not change under rotations, and consequently $\xi$ only depends on the distance $\vartheta \equiv\left|\boldsymbol{\alpha}-\boldsymbol{\alpha}^{\prime}\right|$. The averaging brackets $\langle\ldots\rangle$ denote ensemble averages over statistically equivalent realisations of the random field $\epsilon(\boldsymbol{\alpha})$.

\subsection{Alignment angles and types}

There are two common alignments of ellipticities and position angles that are considered theoretically or observationally: the alignment of the shapes of two objects, or the alignment of the shape of an object with the position of another object. In both cases it is common to relate any ellipticity to a reference axis such as the line connecting a pair of objects, which makes this measurement independent of the coordinate system used. The two ellipticity components 


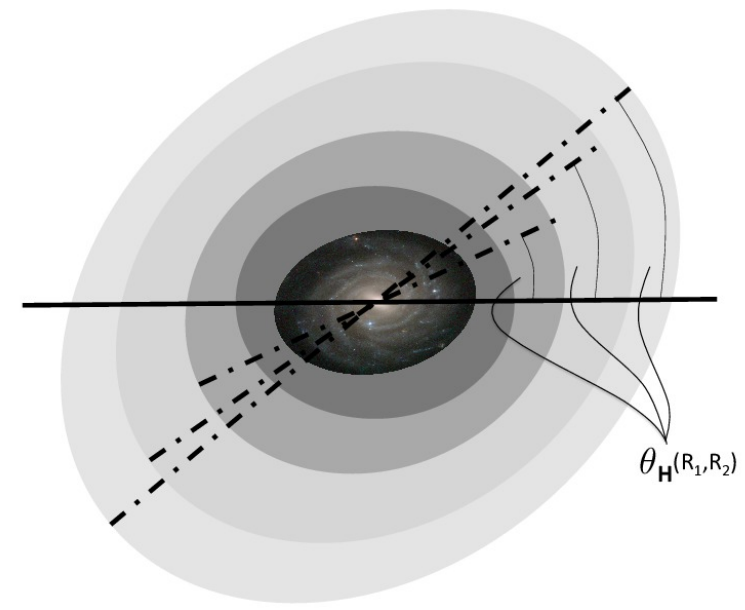

Fig. 2.-: (Mis)alignment of the dark matter halo as a function of radius $\left(\mathrm{R}_{1}, \mathrm{R}_{2}\right)$. The long, horizontal solid line shows the major axis of the innermost radial bin (dark grey); other dashed lines show major axes measured at larger radii. See Table 1 for a complete list of alignments. Galaxy image credit: ESA/Hubble E NASA.

can then be defined as

$$
\begin{aligned}
& \epsilon_{+}=-|\epsilon| \cos \left[2\left(\varphi-\varphi_{0}\right)\right] ; \\
& \epsilon_{\times}=-|\epsilon| \sin \left[2\left(\varphi-\varphi_{0}\right)\right],
\end{aligned}
$$

where $|\epsilon|$ is the absolute value of the complex ellipticity, $\varphi$ the polar angle of the ellipticity, and $\varphi_{0}$ the polar angle of the reference axis (both polar angles are measured in the same coordinate system). $\epsilon_{+}$measures the ellipticity component at $0^{\circ}$ and $90^{\circ}$ from the reference axis, while $\epsilon_{\times}$measures the ellipticity component at $45^{\circ}$ and $135^{\circ}$ from it. In this particular convention, $\epsilon_{+}>0$ represents tangential alignment, and $\epsilon_{+}<0$ radial alignment, with respect to a line connecting the galaxy to a reference point, e.g. the centre of a galaxy cluster. This is illustrated in Figure 1 . There are a variety of sign conventions in the literature. Since gravitational lensing tends to cause tangential alignment and intrinsic alignments are primarily radial, some studies will define the signs such that intrinsic alignments are negative and others such that they are positive. Works that focus on intrinsic galaxy alignments tend to use the latter sign convention to yield a positive signal for the expected radial alignments, e.g. in Figure 8 this latter convention was used.

In Figures 2 to 4 a selection of the possible alignments are represented, while additional alignments not explicitly shown in the figures are listed in Table 1 (found in Section 4.1). Not all combinations of alignments are useful to measure, so if a possible alignment is absent, it is likely not measured in simulations or observations. Alignments of the spin axis are not shown in Figures 2 and 3 or in Table 1, but they can be inferred easily from the figures.

Alignments can be considered on a wide range of scales. In the following discussion, Figures 2 to 4 and Table 1, (mis)alignment angles within a single halo, $\theta$, or between two haloes, $\Theta$, are given two sub-indices for either shape (upper case letter) or position (lower case letter), for a central galaxy (C), satellite galaxy (S), satellite distribution ${ }^{5}$ (B), dark matter halo (H) or a full galaxy sample (G; which includes both satellite and central galaxies), wall (W), filament (F) or the position of the centre of a void (v). The first and second of these indices denote the object in the first and second columns of Table 1, respectively.

The large-scale structure of the Universe can be classified into four separate components called cosmic web elements - typically it is divided in to clusters, filaments, sheets/walls ${ }^{6}$ and voids. In the most basic classification of

\footnotetext{
${ }^{5}$ Note that the terms satellite and satellite distribution are interchangeable with subhalo and subhalo distribution when measuring dark matter alignments.

${ }^{6}$ The terms "sheet" and "wall" are used interchangeably throughout the literature and this review.
} 


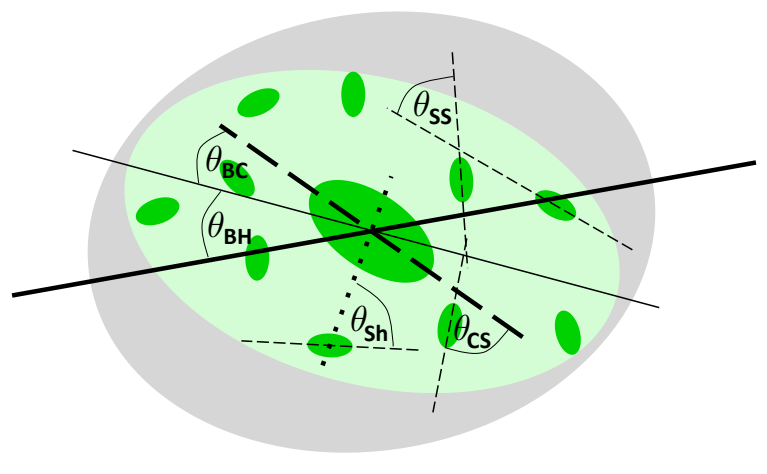

(a) one-halo alignments.

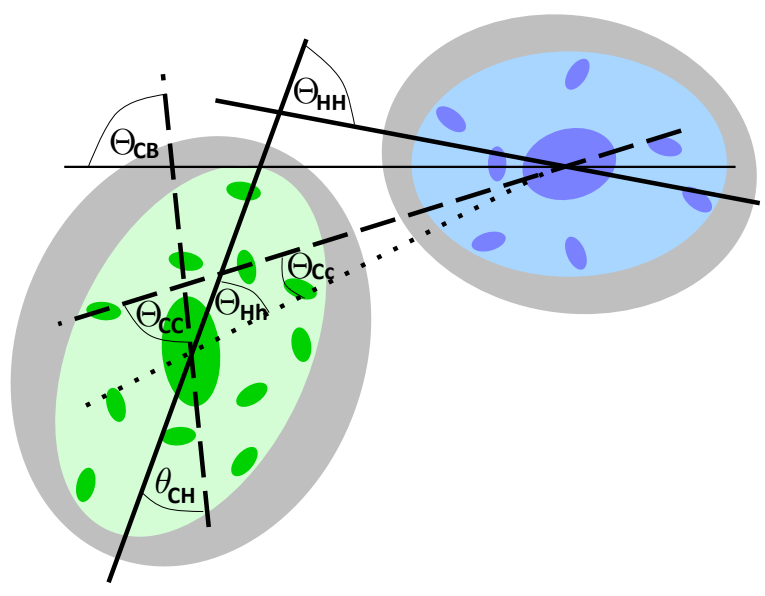

(b) two-halo alignments.

Fig. 3.- : (Mis)alignments, $\theta$, in one- and $\Theta$, in two- haloes. Both: Thick solid and dashed lines show the semi-major axes of the dark matter haloes (grey ellipses; $\mathrm{H}$ ) and central galaxies (larger dark green or blue ellipses; $\mathrm{C}$ ). Thin solid lines show the semi-major axes of the satellite distribution (light green or blue ellipses; B). Left: One-halo - The thin dashed lines show the semi-major axes of the satellites (small dark green ellipses; $\mathrm{S}$ ). The thick dotted line shows the vector connecting the satellite and the centre of the dark matter halo. Right: Two-halo - The dotted line shows the vector connecting two central galaxies. See Table 1 for a complete list of alignments.

the large-scale structure, sheets are planes of structure that delineate the edge of voids, which are underdense and often modelled as spherical or ellipsoidal. Filaments are cylindrical structures (to first approximation) and clusters, the nodes where filaments meet, are modelled as ellipsoidal. A more quantative description of web classification is given in Section 4.1.4.

Figure 2 shows the internal alignments of a dark matter halo at different radii, $\theta_{\mathrm{H}}\left(\mathrm{R}_{1}, \mathrm{R}_{2}\right)$, where $\mathrm{R}_{i}$ is the radius of the halo shells. In this example, the orientation of the inner dark matter halo is being determined with respect to the shells at larger radii. This 3D alignment is measured in simulations and the radius may be an absolute radius relative to the halo centre or an isodensity radius; see Section 4.2.2. Dark matter haloes exist over a huge range of scales, but for the purposes of this work, the scales that are interesting range from subhaloes $\left(\sim 10^{10} \mathrm{M}_{\odot}\right)$ through to clusters $\left(\gtrsim 10^{14} \mathrm{M}_{\odot}\right)$. The dominant, most massive galaxy near the centre of a galaxy sized dark matter halo is known as the central galaxy.

It is common for a dark matter halo with a central galaxy to contain a number (varying between zero and tens) of smaller satellite galaxies (each surrounded by a dark matter subhalo) as shown in Figure 3a. These are less massive than the central galaxy and reside in positions throughout the host halo, rather than at the centre. In this figure the smaller ellipses can be considered either a satellite galaxy or a subhalo, depending on the measurement of interest. On larger scales, dark matter group and cluster haloes contain many tens and hundreds of galaxy sized systems. In this case, the objects inside the larger halo (centrals, satellites, subhaloes and galaxy-sized dark matter haloes) are all considered as substructure within the larger halo.

The shape of the satellite distribution is defined by identifying the positions of the satellites within the halo and fitting a shape (typically an ellipsoid) to their distribution. In this case, the shape of the individual satellites play no role in defining the shape of the satellite distribution. Figure $3 b$ shows alignments within and between two haloes. The shape of the satellite distribution is sometimes used as a proxy for the shape of the dark matter halo observationally. Consequently, $\Theta_{\mathrm{Bb}}$ and $\Theta_{\mathrm{BB}}$ are considered equivalent to $\Theta_{\mathrm{Hh}}$ and $\Theta_{\mathrm{HH}}$ (although the simulation literature does find differences in the shape and orientation of the satellite distribution compared with the dark matter, see Sections 4.2.3, 5.1.2 and 5.2.2). 


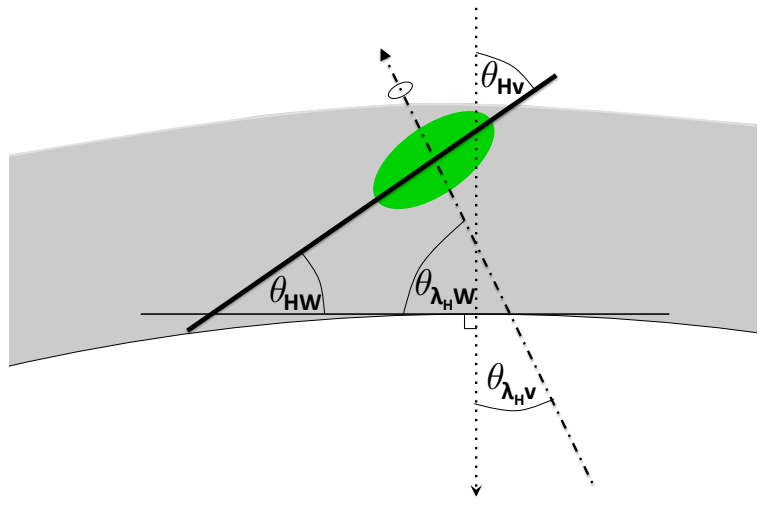

(a) Sheet/Wall \& Void alignments.

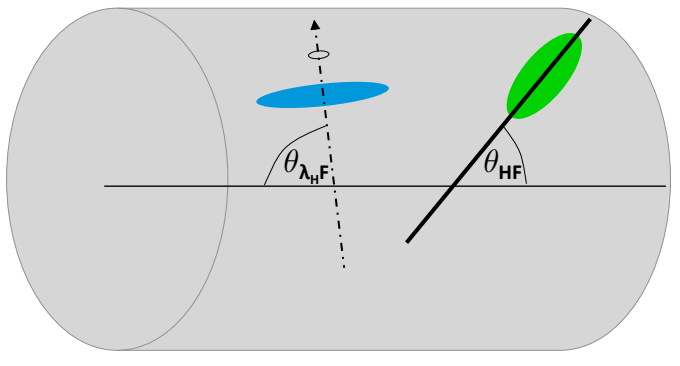

(b) Filament alignments.

Fig. 4.-: (Mis)alignments, $\theta$, in a sheet/wall (W), void (v) and filament $(\mathrm{F})$. The thick lines show the semi-major axes of the dark matter haloes (green and blue ellipses; $\mathrm{H}$ ), thin lines show the plane of the wall or the shape of the filament (grey; W, F), the dot-dash line shows the spin vector of the halo, $\lambda_{\mathrm{H}}$, and the dotted line shows the direction to the centre of the void (v). Left: The sheet/wall is located at the boundary of a spherical void in this figure. When considering a void that is very large, locally the sheet/wall can be treated as flat. The plane of the sheet/wall is oriented left to right on the page (represented by the thin, horizontal line) and normal to the page surface. The vector normal to the plane of the sheet/wall is oriented top to bottom on the page and is represented by the dashed line (that also shows the direction to the centre of the void). See Table 1 for a complete list of alignments.

Figure 4 shows alignments between dark matter haloes, walls, voids and filaments (the clusters are simply massive dark matter haloes, see Figure 3). Also shown in these figures is the orientation of the dark matter halo angular momentum vector, $\lambda_{\mathrm{H}}$, with the cosmic web elements. The angular momentum vector denotes the axis around which the halo is rotating and is discussed further in Sections 3.1.3 and 4.1.3.

\subsection{Ellipticity 2-point correlations}

When considering sets of objects for which ellipticity correlation functions are to be measured, components of the ellipticity, $\epsilon_{+}$and $\epsilon_{\times}$, are typically defined in a coordinate frame aligned with the separation vector, $\boldsymbol{\vartheta}$, between the two galaxies. This involves rotating the two original components of ellipticity in the sky frame ( $\epsilon_{1}$ and $\epsilon_{2}$; see Equation 4). The correlation functions $\xi_{+}$and $\xi_{-}$of these two new ellipticity components are defined as

$$
\xi_{ \pm}(\vartheta)=\left\langle\epsilon_{+} \epsilon_{+}^{\prime}\right\rangle(\vartheta) \pm\left\langle\epsilon_{\times} \epsilon_{\times}^{\prime}\right\rangle(\vartheta),
$$

while the third correlation $\left\langle\epsilon_{+} \epsilon_{\times}^{\prime}\right\rangle(\vartheta)=\left\langle\epsilon_{\times} \epsilon_{+}^{\prime}\right\rangle(\vartheta)$ is parity-odd and expected to vanish due to the parity symmetry of the Universe.

Considering the variance of Fourier modes of the random field, defined in Section 2.1, is a particularly useful concept for homogeneous random fields, because in these cases, the Fourier modes with different wave vectors are uncorrelated, while the variance of equal wave vectors is related to the power spectrum. Constructing power spectra (originally used in the context of polarisation correlations in the cosmic microwave background; see Kamionkowski et al. 1997; Seljak 1997) of the projected galaxy ellipticity field $\epsilon(\boldsymbol{\alpha})$ yields two parity eigen-modes for the angular power spectrum as a function of multipole $\ell$,

$$
C_{E, B}^{\epsilon}(\ell)=\pi \int \vartheta \mathrm{d} \vartheta\left[\xi_{+}(\vartheta) J_{0}(\ell \vartheta) \pm \xi_{-}(\vartheta) J_{4}(\ell \vartheta)\right],
$$

with the Bessel functions of the first kind, $J_{0}$ and $J_{4}$. The physical interpretation of these parity eigen-modes is illustrated in Figure 1. 
Parity-odd $B$-modes are a typical feature of some alignment models, in particular of the quadratic alignment model applicable for spiral galaxies (see Section 3.1.3 for information on the quadratic alignment model). $B$-modes are to lowest order not present in linear alignment models, but can be generated by introducing weighting to the ellipticity field, for instance related to galaxy biasing or peculiar motion. $B$-mode generation through weighting is also well known in higher-order corrections to weak gravitational lensing, which is $B$-mode free to lowest order, but corrections related to geodesic corrections or to clustering can evoke $B$-mode patterns, which are typically small, amounting to a signal of $\sim 10^{-4}$ relative to the $E$-modes on small angular scales, where the effect is strongest (Cooray \& Hu 2002; Bernardeau et al. 2010; Krause \& Hirata 2010).

While the formalism has been outlined for angular correlations of fields at two different positions on the sky, it generalises to correlations of shapes in three dimensions in a straightforward way. Commonly, the correlations of the aligning fields are formulated in three dimensions (using physical separations $\boldsymbol{r}$ derived using known redshifts), though still using the projected (2D) shape. For example, $\xi_{g+}(\boldsymbol{r})$ defines the correlation function of projected galaxy ellipticities with the positions of galaxy overdensities as a function of 3D separation $\boldsymbol{r}$. However, note that the assumption of isotropy is not a very good one in this case, since it is the unobserved 3D shapes that should correlate with an equal strength to galaxies at separate $\boldsymbol{r}$, not the projected 2D shapes. Moreover, at the stage of expressing the relation between redshifts and distances, complications such as redshift-space distortions enter, causing misestimates of radial distances. Observationally, it is difficult to model the impact of redshift space distortions on 2-point correlation functions, particularly on scales where non-linear density perturbations are important (see, e.g., Kaiser 1987 for a review of redshift space distortions in general, or Singh et al. 2015 for a derivation of their lowest-order impact on intrinsic alignment 2-point correlations). Without a good model, the three dimensional ellipticity correlation functions are difficult to interpret. To avoid both of these complications (anisotropy and redshift-space distortions), a two-dimensional ellipticity correlation function for galaxies as a function of their transverse separation on the sky $r_{p}$ is commonly calculated,

$$
w_{g+}\left(r_{p}\right)=\int_{-\Pi_{\max }}^{+\Pi_{\max }} \xi_{g+}\left(r_{p}, \Pi\right) \mathrm{d} \Pi,
$$

determined by projecting the equivalent three-dimensional correlation function $\xi_{g+}=\left\langle\epsilon_{+} g\right\rangle$ between ellipticity and the galaxy density along the line-of-sight ( $\Pi$ is the separation in the redshift direction). This projected correlation function can similarly be found for other combinations of observables including the ellipticity components $+, \times, \epsilon, g$, the galaxy overdensity with respect to the mean, $g=\rho_{g} / \bar{\rho}_{g}-1$, and $\delta$, the matter overdensity with respect to the mean, $\delta=\rho / \bar{\rho}-1$. The densities $\rho_{g}$ and $\rho$ are the galaxy density and the matter density respectively.

Identifying intrinsic galaxy alignments is particularly important for upcoming weak lensing surveys, because they mimic the coherent shape distortions resulting from gravitational shear. A correlator of two galaxy ellipticities can be taken directly from the correlation in Equation (1),

$$
\underbrace{\left\langle\epsilon \epsilon^{\prime}\right\rangle}_{\text {observed }}=\underbrace{\left\langle\gamma \gamma^{\prime}\right\rangle}_{\mathrm{GG}}+\underbrace{\left\langle\epsilon^{\mathrm{s}} \epsilon^{\mathrm{s}}\right\rangle}_{\mathrm{II}}+\underbrace{\left\langle\gamma \epsilon^{\prime \mathrm{s}}\right\rangle+\left\langle\epsilon^{\mathrm{s}} \gamma^{\prime}\right\rangle}_{\mathrm{GI}} .
$$

The left side of the equation is the correlator of the observed ellipticities. GG is the gravitational lensing shear correlation (the signal that is most important in weak lensing analyses; see Joachimi et al. 2015 for a more comprehensive introduction to weak gravitational lensing). II is the correlation between the intrinsic shapes of two galaxies, and GI is the correlation between the gravitational shear of one galaxy and the intrinsic shape of the other galaxy. Only one of the GI terms is non-zero because any gravitational shear associated with a galaxy closer to the observer can not be correlated with the intrinsic shape of a galaxy further away from the observer ${ }^{7}$. In addition to shape correlations, intrinsic alignments can cause cross correlations between the shape and the local density, which we refer to as $\delta \mathrm{I}$-correlations in this review and these give rise to GI correlations.

\footnotetext{
${ }^{7}$ This may not be true in the presence of photometric redshift errors, where the relative positions of galaxies along the line of sight may be confused.
} 


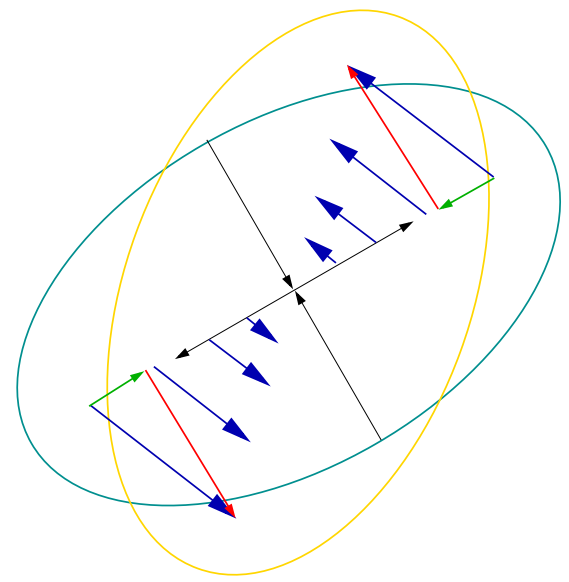

(a) Tidal Torquing

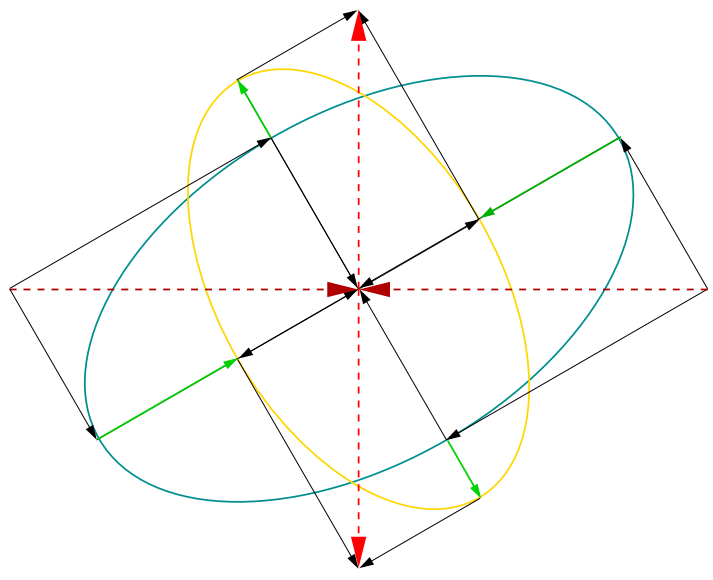

(b) Tidal Stretching

Fig. 5.—: Illustration of tidal stretching and torquing. Left: Embedding the halo (blue ellipse) into a tidal field which varies across the halo (blue arrows) gives rise to two effects: First, there is a change in shape of the halo caused by the components of the tidal shear inside the principal axis system of the halo, leading to an anisotropic change in shape (green arrows). Second, there is a shearing deformation of the halo (red arrows), which generates angular momentum. Right: If a halo (blue ellipse) is embedded into a tidal gravitational field, it is tidally stretched to a new shape (yellow ellipse): The tidal shear displacement (red arrows) is projected into the coordinate frame defined by the halo principal axes (black arrows), such that the resulting tidal fields cause a contraction of the halo in one direction and a expansion of the halo in the other (green arrows).

\section{Theory and modelling}

The alignments of galaxies with cosmic structures is a phenomenon that occurs on a variety of scales. In general these alignments are thought to be sourced through tidal interactions of galaxies with the gravitational field of larger structures. Fundamentally, alignments fall into three regimes: On very large scales above $\sim 10 \mathrm{Mpc}$, galaxies are tidally aligned with the linearly evolving cosmic large-scale structure (Section 3.1). On scales below $\sim 1 \mathrm{Mpc}$, galaxies may align themselves with their host halo and this particular alignment can be described with the halo model (Section 3.2). Intermediate scales of a few Mpc are difficult to grasp due to non-linearly evolving structures and effects due to clustering and a strong peculiar motion contribution to the galaxy redshifts; how to model the influence of these effects on galaxy alignments is a topic of current research (Section 3.3).

There are two theories commonly employed to explain how tidal interactions determine the alignment and hence the shape of a galaxy: stellar ellipsoids of elliptical galaxies may be tidally distorted (Section 3.1.2), and the orientation of stellar discs in spiral galaxies may be determined by the angular momentum direction, which in turn follows from tidal interactions (Section 3.1.3). While tidal interaction processes on large scales may be be described by perturbation theory (Section 3.1.1), the shape and orientation of the stellar distribution inside a halo is a more complicated problem that requires numerical simulations to solve (see Sections 4 to 6). It should be emphasised that alignment models, which mediate between an aligning large-scale field and an observable galaxy shape, may be based on fields other than the tidal gravitational fields, for example vorticity.

\subsection{Large-scale alignments}

\subsubsection{Tidal interactions of haloes}

Tidal interactions of galaxies with the cosmic large-scale structure can be modelled as a perturbative process. Consider the position, $x_{i}(\boldsymbol{q}, a)$, of a particle as a function of scale factor, $a$, and initial position, $\boldsymbol{q}$. To lowest order, the positions follow straight lines over time, along a direction determined by the gradient, $\partial_{i} \Psi$, of the displacement 


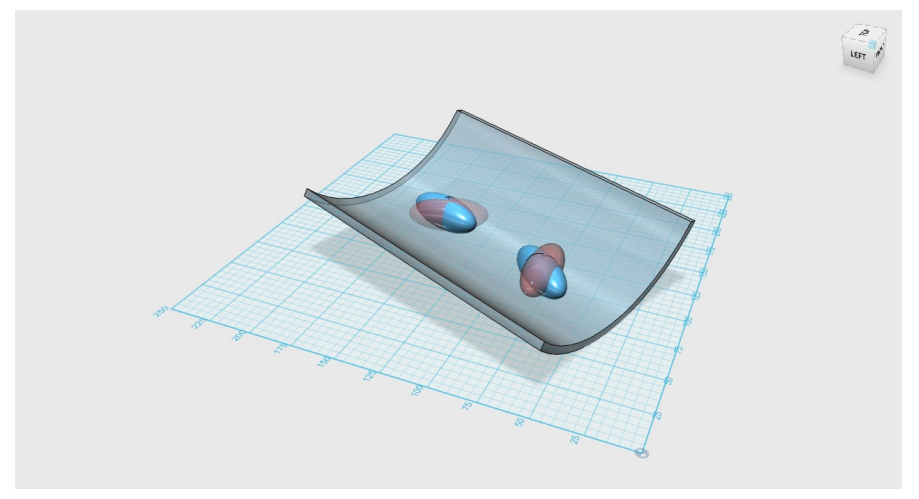

Fig. 6.-: Two elliptical galaxies embedded in a gravitational potential (grey sheet). They experience correlated tidal shears and react to the tidal fields by changing their initially uncorrelated shapes (red), to correlated shapes (blue).

potential, $\Psi$, (Zel'dovich 1970),

$$
x_{i}(\boldsymbol{q}, a)=q_{i}-D_{+}(a) \partial_{i} \Psi(\boldsymbol{q}),
$$

where $D_{+}(a)$ is the growth function. The interaction of an entire protohalo is obtained by Taylor-expanding the trajectories around the centre of gravity, $\overline{\boldsymbol{q}}$,

$$
x_{i}(\boldsymbol{q}, a) \approx q_{i}-D_{+}(a)\left(\partial_{i} \Psi(\overline{\boldsymbol{q}})+\sum_{j}\left(q_{j}-\bar{q}_{j}\right) \partial_{i} \partial_{j} \Psi(\overline{\boldsymbol{q}})\right),
$$

which reveals the bulk motion of the halo along $\partial_{i} \Psi(\overline{\boldsymbol{q}})$ and the differential motion of the particles around the centre, which is encoded in the tidal field tensor, $\partial_{i} \partial_{j} \Psi(\overline{\boldsymbol{q}}) \equiv T_{i j}(\overline{\boldsymbol{q}})$. The displacement potential is related to the Newtonian gravitational potential, $\Phi$, by a factor of $4 \pi G$, where $G$ is Newton's gravitational constant. It should be noted that in this picture the protohalo is treated as a test object embedded into a tidal field, whereas in reality the tidal fields themselves determine which particles will ultimately compose the halo.

The haloes principal axis frame defines a coordinate frame into which the tidal field, $\partial_{i} \partial_{j} \Phi$, can be decomposed. If the tidal field tensor does not coincide with the principle axis frame of the halo, a shearing motion is exerted onto the halo, which ultimately leads to angular momentum generation, as shown by Figure 5a. As long as merging or accretion do not play an important role, the galactic disc can be expected to form perpendicular to the halo angular momentum direction: This is the picture behind the quadratic alignment of spiral galaxies.

The component of the tidal field coinciding with the haloes principal axis frame gives rise to an anisotropic deformation as illustrated by Figure 5b. This effect is used to explain the alignment of elliptical galaxies with the large-scale structure and is the basis of the linear alignment model for elliptical galaxies, and even though the haloes and elliptical galaxies are collapsed objects, it is still reasonable to expect a deformation of the structures with the tidal shear field.

\subsubsection{Linear alignment model for elliptical galaxies}

Elliptical galaxies are supported by the velocity dispersion of the dark matter particles and of the stars, which can be assumed to be in virial equilibrium. If an elliptical galaxy is embedded in a tidal gravitational field, it will distort the potential of the galaxy's dark matter halo and force the distribution of stars to assume a new equilibrium configuration, resulting in a new shape as illustrated by Figure 6. The reaction of an elliptical galaxy to an external tidal field can be considered instantaneous because the dynamical time scale of stars inside the galaxy should be short compared to the time scale on which tidal fields change due to structure formation, such that the distortion of the stellar quadrupole grows with the growth function $D_{+}$. This picture assumes that the shape of an elliptical galaxy is perturbed by an external tidal shear field while it is not influenced by e.g. merging. 
The intrinsic complex shear, $\gamma^{I}$ (see Joachimi et al. 2015 for an introduction to $\gamma^{I}$ ), in this model is proportional to the tidal shear field projected onto the sky,

$$
\gamma^{I}=-\frac{C_{1}}{4 \pi G}\left(\partial_{x}^{2}-\partial_{y}^{2}+2 \mathrm{i} \partial_{x y}^{2}\right) \mathcal{S}(\Phi),
$$

where $G$ is Newton's gravitational constant and $x$ and $y$ are Cartesian coordinates in the plane of the sky. Smoothing of the gravitational potential, as indicated by $\mathcal{S}(\Phi)$, on the scale of the halo ensures that the halo as a whole reacts to tidal fields and avoids substructures that would be introduced by small-scale fluctuations of $\Phi$. The derivatives $\left(\partial_{x}^{2}-\partial_{y}^{2}\right) \mathcal{S}(\Phi)$ and $\left(2 \mathrm{i} \partial_{x y}^{2}\right) \mathcal{S} \Phi$ (with the prefactor) give the tangential and cross components of the shear with respect to the $x$ axis. $C_{1}$ is a constant of proportionality containing information on the strength of the reaction that a galaxy experiences as it adjusts its shape due to an external tidal shear field. Bridle \& King (2007) measured this through ellipticity correlations in SuperCOSMOS data (Brown et al. 2002) and found $C_{1} \simeq 5 \times 10^{-14}\left(h^{2} \mathrm{M}_{\odot} \mathrm{Mpc}^{-3}\right)^{-1}$. The relation between ellipticity and tidal shear is, in the limit of weak fields, a linear one and it is from this linearity that linear alignments derive their name (Catelan et al. 2001). Physically, the dependence of the distorted galaxy shape on the second derivatives of the gravitational potential, corresponds exactly to the tidal stretching illustrated in Figure 5b. In addition, assuming a linear relationship between the quadrupole of the brightness distribution, which is a symmetric tensor of rank 2, and the tidal shear transverse to the line of sight, which is a tensor of the same type, is reasonable motivation for Equation 11 (Hirata \& Seljak 2004, 2010) .

Hirata \& Seljak (2004) related the gravitational potential at an early time in the galaxy's evolution to the matter density contrast, $\delta$, on linear scales via the Poisson equation,

$$
\Phi(\boldsymbol{k})=-4 \pi G \frac{\bar{\rho}(z)}{\bar{D}(z)} a^{2} k^{-2} \delta(\boldsymbol{k}),
$$

where $\bar{D}(z) \propto(1+z) D_{+}(z)$ is the scaled growth factor that is normalized to unity during matter domination, $a$ is the scale factor, $k$ is the wave vector and $k$ is the wavenumber of the wave vector.. The mean background density, $\bar{\rho}(z)=\Omega_{m}(z) \rho_{\text {crit }}(z)$, where $\rho_{\text {crit }}(z)$ is the critical density of the Universe, sets the strength of the gravitational potential. The critical density of a smoothed background that produces a spatially flat Universe is,

$$
\rho_{\text {crit }}(z)=\frac{3 H(z)^{2}}{8 \pi G},
$$

where $H(z)$ is the Hubble constant at redshift $z$.

Hirata \& Seljak (2004) assumed that the 'primordial' tidal gravitational field acting during the formation of the galaxy determines the galaxy alignment, which would then be frozen in during the subsequent evolution. As this model is mostly applicable to elliptical galaxies, which are believed to undergo dramatic changes in their morphology during mergers at relatively recent times, it may be more valid to instead assume a quasi-instantaneous response to the local tidal field at any given time, which would lead to a different redshift scaling of the alignment signal amplitude. However, the redshift dependence predicted by Hirata \& Seljak (2004), which we adopt in the following, is consistent with current observations (Joachimi et al. 2011).

Irrespective of whether the density field is in a linear or non-linear stage of evolution, the link between the observable $\gamma^{I}$ to the matter density power spectrum $P_{\delta \delta}^{\text {lin }}(k)$, is best formulated in Fourier space. As information on the tidal shear field is only available at positions where there are galaxies, the density-weighted intrinsic shear, $\tilde{\gamma}^{I}$, is the natural observable. In the complex notation this is given by

$$
\tilde{\gamma}^{I}(\boldsymbol{k})=\frac{C_{1} \bar{\rho}}{\bar{D}} a^{2} \int \frac{\left(k_{2 x}^{2}-k_{2 y}^{2}+2 \mathrm{i} k_{2 x} k_{2 y}\right)}{k_{2}^{2}} \delta\left(\boldsymbol{k}_{2}\right)\left[\delta_{D}^{(3)}\left(\boldsymbol{k}_{1}\right)+\frac{b_{g}}{(2 \pi)^{3}} \delta\left(\boldsymbol{k}_{1}\right)\right] \mathrm{d}^{3} \boldsymbol{k}_{1},
$$

where $\boldsymbol{k}_{\mathbf{2}}=\boldsymbol{k}-\boldsymbol{k}_{1}, \delta_{D}^{(3)}$ is the 3D Dirac delta function and $b_{g}$ is the linear biasing factor, which relates the relative fluctuation in the number density of galaxies to the local dark matter density. Note that the multiplicative density

${ }^{8}$ Note that Hirata \& Seljak (2010) is an updated version of the original Hirata \& Seljak (2004) paper that fixes an error in a conversion factor that propagated through several equations. 
weighting becomes a convolution in Fourier space with $\boldsymbol{k}_{1}$ as the integration variable, such that the observable $\tilde{\gamma}^{I}$ depends on $\boldsymbol{k}$. The derivatives of the gravitational potential translate in Fourier space into multiplications with the corresponding components of the wave vector, in this case $\boldsymbol{k}_{2}$. In this way, the resulting $E$-mode part of the II power spectrum $P_{\mathrm{II}}(k)$ of $\tilde{\gamma}^{I}$ is

$$
P_{\mathrm{II}}(k)=\frac{C_{1}^{2} \bar{\rho}^{2}}{\bar{D}^{2}} a^{4}\left\{P_{\delta \delta}^{\operatorname{lin}}(k)+b_{g}^{2} \int\left[f_{E}\left(\boldsymbol{k}_{2}\right)+f_{E}\left(\boldsymbol{k}_{1}\right)\right] \times f_{E}\left(\boldsymbol{k}_{2}\right) \frac{P_{\delta \delta}^{\mathrm{lin}}\left(k_{1}\right) P_{\delta \delta}^{\mathrm{lin}}\left(k_{2}\right)}{(2 \pi)^{3}} \mathrm{~d}^{3} \boldsymbol{k}_{1}\right\},
$$

where $f_{E}(\boldsymbol{k})$ is a geometric function that singles out correlations between the $E$-modes of the ellipticity field (Hirata $\&$ Seljak 2004). Note that the $B$-mode correlations are zero, due to the symmetry of the tidal shear tensor. $B$-modes can be introduced in the context of a linear alignment model by e.g. clustering in analogy to second-order effects in gravitational lensing.

The second term in brackets in Equation (15) is caused by the density weighting and is proportional to the square of the linear matter power spectrum, $P_{\delta \delta}^{\operatorname{lin}}\left(k_{1}\right) P_{\delta \delta}^{\text {lin }}\left(k_{2}\right)$. It is therefore sub-dominant compared to the first term on large scales and is usually ignored in the literature when the linear alignment model is applied. Being linear in the tidal field, there is a cross-correlation between alignments and weak gravitational lensing (Hirata \& Seljak 2004), even for Gaussian initial conditions, which persists for nonlinearly evolving fields. While the correlation between shapes in the linear alignment model is necessarily positive, the cross-correlation between the overdensity and the density-weighted intrinsic shear is in general negative because mass overdensities tangentially align the lensed images of background objects while radially aligning local galaxies if the overdensities are massive enough to define the principal axis of the local tidal quadrupole. The corresponding power spectrum reads

$$
P_{\delta \mathrm{I}}(k)=-\frac{C_{1} \bar{\rho}}{\bar{D}} a^{2} P_{\delta \delta}^{\operatorname{lin}}(k)
$$

which is important to both weak cosmic shear (Hirata \& Seljak 2004; Hirata et al. 2004) and galaxy-galaxy lensing (Blazek et al. 2012). The parameter $C_{1}$ will, in general, depend on galaxy properties including luminosity, mass, and formation time.

Correlations of the linear type can be quite long-ranged: Ellipticity auto-correlations (II) have been measured in the Sloan Digital Sky Survey (SDSS; York et al. 2000) to reach out to $30 \mathrm{~h}^{-1} \mathrm{Mpc}$ (Okumura et al. 2009), and crosscorrelations between shape and density to almost $100 \mathrm{~h}^{-1} \mathrm{Mpc}$ (e.g. Mandelbaum et al. 2006b, and see also Kirk et al. 2015 for a comprehensive list of observations). The shape-density correlations give rise to intrinsic shape-lensing (GI) correlations (see Equation 8), and these have been marginally detected in the Canada-France-Hawaii Telescope Lensing Survey (CFHTLenS; Heymans et al. 2013; MacCrann et al. 2015).

The linear alignment model is characterized by a single parameter (which may depend on properties like luminosity, mass, etc.) that sets the strength of the external tidal field in relation to the ellipticity of the distorted galaxy. Recent analytical studies found that tidal stretching on a stellar structure in equilibrium may not be strong enough to explain the observed alignments of elliptical galaxies (Camelio \& Lombardi 2015). It should be noted that even for the case where the particular model for tidal stretching is incorrect, it may still effectively describe other alignment mechanisms due to its generality.

\subsubsection{Quadratic alignment model for spiral galaxies}

Commonly, spiral galaxy alignments are explained by the alignment of their angular momentum with the tidal field of the large-scale structure, which occurs due to tidal torquing. If the symmetry axis of the galactic disc follows the angular momentum direction of the host halo, the observer will measure ellipticities which depend on the angle of inclination of the galactic disc. In the case where the angular momentum points toward the observer, the galaxy is viewed face on and will have a small ellipticity, in contrast to the case where the angular momentum is perpendicular to the line of sight, implying that the galactic disc is viewed edge on and will have a high ellipticity. In alternative models, the alignment of spiral galaxies with the large-scale structure is traced back to the vorticity of the surrounding flow field, to the accretion pattern of matter flows converging on the galaxy. 
The main uncertainties of this model are the extent to which the angular momentum can be predicted perturbatively by tidal torquing and the orientation of the stellar disc relative to the angular momentum direction of the host halo. In fact, there may be strong deviations in the latter related to merging, which can reorient the angular momentum, and dissipative processes, which can destroy angular momentum. Tidal torquing, however, has been shown to predict the angular momentum direction of haloes reasonably well, which matters in this context, but would fail at predicting the correct angular momentum magnitude (Catelan \& Theuns 1996).

The tidal torquing mechanism combines halo inertia and gravitational tidal shear to generate angular momentum, meaning that the angular momentum resulting from the same tidal fields can be different depending on the halo shape. In order to capture this, Lee \& Pen (2000) introduced a model that gives a Gaussian distribution $p(\boldsymbol{J} \mid T)$ of angular momenta $\boldsymbol{J}$,

$$
p(J \mid T)=\frac{1}{\sqrt{(2 \pi)^{3} \operatorname{det}(C)}} \exp \left(-\frac{1}{2} \sum_{\alpha, \alpha^{\prime}} J_{\alpha}\left(C^{-1}\right)_{\alpha \alpha^{\prime}} J_{\alpha^{\prime}}\right),
$$

conditional on the tidal shear $T$. The conditionality of the Gaussian distribution is incorporated in the covariance matrix $C_{\alpha \alpha^{\prime}}=\left\langle J_{\alpha} J_{\alpha^{\prime}}\right\rangle$, which also depends on the tidal shear,

$$
\left\langle J_{\alpha} J_{\alpha^{\prime}}\right\rangle=\frac{\left\langle\boldsymbol{J}^{2}\right\rangle}{3}\left(\frac{1+a_{\mathrm{T}}}{3} \delta_{\alpha \alpha^{\prime}}-a_{\mathrm{T}} \sum_{\sigma} \hat{T}_{\alpha \sigma} \hat{T}_{\sigma \alpha^{\prime}}\right),
$$

where $\delta_{\alpha \alpha^{\prime}}$ is the Kronecker $\delta$. The model is characterised by the misalignment parameter, $a_{\mathrm{T}}$, which allows the variation between random angular momenta for $a_{\mathrm{T}}=0$ to maximally aligned angular momenta for $a_{\mathrm{T}}=3 / 5$. Larger values for $a_{\mathrm{T}}$ would be in violation of keeping $C_{\alpha \alpha^{\prime}}$ positive definite.

$\hat{T}$ is the unit-normalised $\left(\hat{T}_{\alpha \sigma} \hat{T}_{\sigma \alpha}=1\right)$, traceless $(\operatorname{tr} \hat{T}=0)$ tidal shear tensor, which can easily be derived using

$$
\tilde{T}_{\alpha \alpha^{\prime}}=T_{\alpha \alpha^{\prime}}-\frac{\operatorname{Tr}(T)}{3} \delta_{\alpha \alpha^{\prime}}
$$

and rescaling $\hat{T}=\tilde{T} /|\tilde{T}|$ with $\tilde{T}=\sqrt{\bar{T}_{\alpha \sigma} \bar{T}_{\sigma \alpha}}$. It should be emphasised that the traceless unit-normalised tensor is derived from the initial conditions for structure formation, which determine the orientation of the eigensystem of $\hat{T}$ and ultimately the angular momentum direction.

Squaring the tidal shear gives rise to short-ranged correlations in the angular momentum directions, and ultimately in the derived galaxy shapes (Schäfer \& Merkel 2012). This picture is illustrated in Figure 7, where two neighbouring haloes are subject to correlated tidal torquing of their motion along the gradient of the gravitational potential. They build up correlated angular momenta, which determines the orientation of their discs and therefore, their shapes. There can be significant differences in the torquing process in filamentary structures relative to the average locations in the cosmic structure, as exemplified by (Codis et al. 2015a), who introduced an anisotropic tidal torquing model for describing these situations.

Under the assumption that the symmetry axis of the galactic disc follows the angular momentum direction of the host halo, it is possible to write down relationships of the form (Catelan et al. 2001):

$$
\begin{aligned}
& \gamma_{+}^{I}=f\left(J, J_{z}\right)\left(J_{x}^{2}-J_{y}^{2}\right) \\
& \gamma_{\times}^{I}=2 f\left(J, J_{z}\right)\left(J_{x} J_{y}\right),
\end{aligned}
$$

where the function $f\left(J, J_{z}\right)$ describes the scaling of the observable intrinsic ellipticity $\gamma^{I}$, with angular momentum magnitude and direction. In general, the observed ellipticity should show a scaling behaviour (Crittenden et al. 2001)

$$
\left|\gamma^{I}\right| \propto \frac{1-\hat{J}_{z}^{2}}{1+\hat{J}_{z}^{2}}
$$

The angular momentum is related to the tidal shear through

$$
J_{\alpha} \propto \epsilon_{\alpha \beta \gamma} \sum_{\sigma} I_{\beta \sigma} T_{\sigma \gamma},
$$




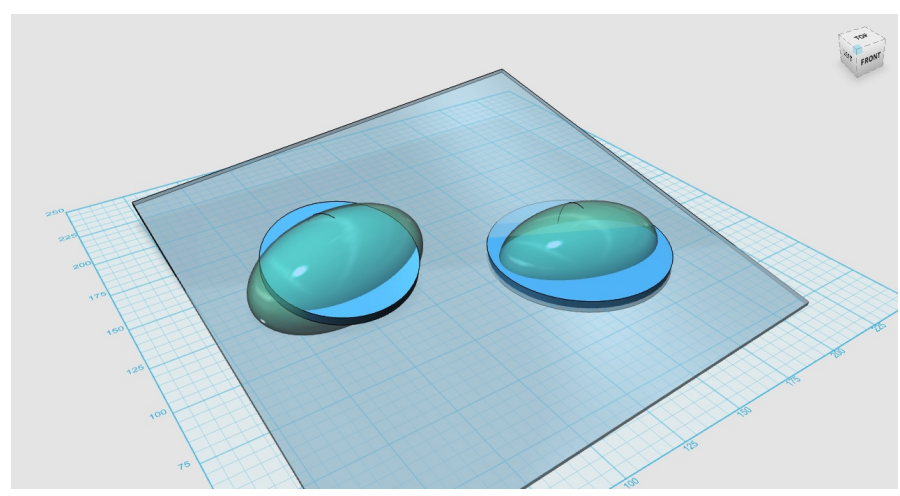

Fig. 7.-: Two haloes (green) are embedded in a gravitational potential (grey sheet). They experience tidal torquing due to differences in the slope of the gravitational potential across their volume, and build up angular momentum by tidal torquing. Correlations in the tidal shear field leads then to correlations in the angular momentum, and ultimately in the orientation of galactic discs (blue), leading to correlated shapes in the observation.

where $I_{\beta \sigma}$ is the tensor of second moments of the mass distribution (the inertia) and $\epsilon_{\alpha \beta \gamma}$ is the antisymmetric symbol (or Levi-Civita symbol) in 3 dimensions (Schäfer \& Merkel 2012). A direct connection of the galaxy shape, in analogy to the linear alignment model, would use the quadratic dependence between shape and tidal shear according to

$$
\gamma^{I}=C_{2}\left(\tilde{T}_{x \mu}^{2}-\tilde{T}_{y \mu}^{2}, 2 \tilde{T}_{x \mu} \tilde{T}_{y \mu}\right),
$$

where the constant of proportionality, $C_{2}$, captures all the processes related to the angular momentum variance for a given tidal shear and of the inclination of the galactic disc. The effective model in Equation (24) uses the traceless shear $\tilde{T}_{\alpha \alpha^{\prime}}$ rather than the tidal shear $T_{\alpha \alpha^{\prime}}$ because alignment of spiral galaxies is an orientation effect where the absolute value of the tidal shears is not relevant, only the orientation of the tidal shear eigensystem. The subscripts $x$ and $y$ refer to the coordinate system in which $\gamma_{+}^{I}$ assumes the components $C_{2}\left(\tilde{T}_{x \mu}^{2}-\tilde{T}_{y \mu}^{2}\right)$ and $2 C_{2} \tilde{T}_{x \mu}$.

Using this model as an effective model for spiral galaxies in analogy to the alignments of ellipticals, the resulting density weighted intrinsic shear $\tilde{\gamma}^{I}$ is (Hirata \& Seljak 2004),

$$
\tilde{\gamma}^{I}(\boldsymbol{k})=\frac{C_{2} \bar{\rho}^{2}}{(2 \pi)^{3} \bar{D}^{2}} a^{4} \int h_{E}\left(\hat{\boldsymbol{k}}_{1}^{\prime}, \hat{\boldsymbol{k}}_{2}^{\prime}\right) \delta\left(\boldsymbol{k}_{1}^{\prime}\right) \delta\left(\boldsymbol{k}_{2}^{\prime}\right)\left[\delta_{\mathrm{D}}^{(3)}\left(\boldsymbol{k}_{3}^{\prime}\right)+\frac{b_{g}}{(2 \pi)^{3}} \delta\left(\boldsymbol{k}_{3}^{\prime}\right)\right] \mathrm{d}^{3} \boldsymbol{k}_{1}^{\prime} \mathrm{d}^{3} \boldsymbol{k}_{2}^{\prime},
$$

where $\hat{\boldsymbol{k}}_{a}=\boldsymbol{k}_{a} /\left|k_{a}\right|$ and $\boldsymbol{k}_{3}^{\prime}=\boldsymbol{k}-\boldsymbol{k}_{1}^{\prime}-\boldsymbol{k}_{2}^{\prime}$. The term proportional to the galaxy bias $b_{g}$ introduces a weighting proportional to the galaxy density, therefore, two of the three powers of $\delta$ are due to the quadratic alignment and one is from the applied density weighting. Similar to the case for linear alignments, there is a geometric factor $h_{E}$ that depends, in this case, on the directions $\hat{\boldsymbol{k}}$ of the wave vector (Hirata \& Seljak 2004). The intrinsic alignment power spectrum $P_{\mathrm{II}}(k)$ for the II correlation takes the form

$$
\begin{aligned}
P_{\mathrm{II}}(k)=\frac{C_{2}^{2} \bar{\rho}^{4}}{\bar{D}^{4}} & a^{8}\left\{2 \int\left[h_{E}\left(\hat{\boldsymbol{k}}_{1} \hat{\boldsymbol{k}}_{2}\right)\right]^{2} \frac{P_{\delta \delta}^{\operatorname{lin}}\left(k_{1}\right) P_{\delta \delta}^{\operatorname{lin}}\left(k_{2}\right)}{(2 \pi)^{3}} d^{3} \boldsymbol{k}_{1}\right. \\
& \left.+\frac{2}{3} b_{g}^{2} \int\left[h_{E}\left(\hat{\boldsymbol{k}}_{1}^{\prime}, \hat{\boldsymbol{k}}_{2}^{\prime}\right)+h_{E}\left(\hat{k}_{2}^{\prime}, \hat{k}_{3}^{\prime}\right)+h_{E}\left(\hat{k}_{3}^{\prime}, \hat{k}_{1}^{\prime}\right)\right]^{2} \frac{P_{\delta \delta}^{\operatorname{lin}}\left(k_{1}^{\prime}\right) P_{\delta \delta}^{\operatorname{lin}}\left(k_{2}^{\prime}\right) P_{\delta \delta}^{\operatorname{lin}}\left(k_{3}^{\prime}\right)}{(2 \pi)^{6}} d^{3} \boldsymbol{k}_{1}^{\prime} d^{3} \boldsymbol{k}_{2}^{\prime}\right\} .
\end{aligned}
$$

In this way, the ellipticity is linked to the tidal shear field through the angular momentum direction and it is possible to trace correlations in the ellipticity field back to those in the tidal shear field, which in turn are related to density correlations by the Poisson equation. This has been demonstrated by Crittenden et al. (2001), who computed $E$ and $B$-mode ellipticity correlation functions and showed that they dominate over the lensing signal at redshifts below 
$z=0.3$, and are a small but significant contribution to weak lensing spectra at redshifts of unity, under the assumption of perfect alignment between the galaxy and halo angular momenta. The main uncertainties are the misalignment parameter $a_{\mathrm{T}}$, which is measured in numerical simulations by comparing the tidal shear acting on a halo with the resulting angular momentum, and the orientation of the galactic disc relative to the angular momentum direction. This relation is poorly understood, in fact many analytical works assume perfect alignment but allow for a finite thickness of the galactic disc, which dilutes the orientation effect and leads to a less pronounced ellipticity variation when tilting the angular momentum direction (e.g. Crittenden et al. 2001, 2002; Capranico et al. 2013; Merkel \& Schäfer 2013). Following the work of Crittenden et al. (2001), Capranico et al. (2013) computed angular ellipticity power spectra and compared these with weak lensing shear power spectra. The intrinsic $E$-mode (II) spectra resulting from the quadratic alignments was found to be an order of magnitude less than the weak lensing power spectra for lensing surveys reaching unit redshift, while the $B$-mode spectra would be an important test of systematics. Generalising on this, Merkel \& Schäfer (2013) computed three-dimensional ellipticity (II) power spectra and found them to differ from weak lensing power spectra in terms of their scale dependence and their correlation properties between different Fourier-modes, which arise in this formalism because the projection onto angular correlations does not assume the Limber-approximation.

The density-intrinsic alignment cross-power spectrum is zero in the simple quadratic alignment model due to the assumed Gaussianity of the density field. The correlation would be proportional to three powers of the density field, two from the alignment model and one from the density field, and is zero, because odd moments of a Gaussian distribution vanish identically. However, there will certainly be some non-zero contribution to the cross-term even in the quadratic alignment model once the third- and higher-order corrections to Equation (24) are taken into account, as well as from non-Gaussianity due to non-linear growth of structure.

\subsection{Alignments on scales smaller than host haloes}

The current understanding of galaxy alignments within haloes is fundamentally hampered by the limited knowledge of the non-linear evolution and baryonic physics that shapes the haloes themselves and the galaxies they host. Without a physical grounding to study intrinsic galaxy alignments within a halo, the best approach is to describe alignments through the halo model.

The halo model (e.g. Seljak 2000; Cooray \& Sheth 2002) is an analytical description of the clustering of dark matter in the Universe, based on the ansatz that all dark matter particles are contained within haloes. It further assumes that the mass of a halo is the physical quantity that drives virtually all properties of the haloes (and of the galaxies that inhabit them). This assumption has motivations heavily rooted in the results of $N$-body simulations. For example, the abundance (see e.g. Tinker et al. 2008), the bias (e.g. Tinker et al. 2010), and the matter density profile (see e.g. Navarro et al. 1997; Dutton \& Macciò 2014) are all functions of the halo mass (albeit with some scatter). Perhaps even more interestingly, properties of galaxies such as stellar mass, luminosity, star formation efficiency and size all depend to first order on the mass of their host haloes. Using this model, predictions for the clustering of dark matter, as well as galaxies, can be as accurate as 5-10 \% (e.g. Giocoli et al. 2010; van den Bosch et al. 2013). The model has been further improved through incorporation of the potential effects of galaxy formation (e.g. baryonic feedback) into the clustering of dark matter and galaxies (see Fedeli 2014; Fedeli et al. 2014). As the observational and simulated data increase in precision, it has become apparent that the halo formation time plays a similarly important role in shaping the properties of galaxies such as halo mass. This effect is commonly known as assembly bias: at fixed halo mass, the scatter in galaxy properties mainly owes to the different assembly histories of haloes (see e.g. Gao \& White 2007; Wang et al. 2013).

With halo mass being the central quantity, other relevant quantities of the halo model are the number density of haloes of a given mass and the relation between mass and observable. With these, it is possible to describe the statistical properties of the observable (taken at a single point), while the correlation functions of the observable need in addition the density profile inside an individual halo (which is taken to scale with halo mass) and the correlation function of the haloes, while assuming that the relation between the observable and the halo mass is a local one. In this approximation, the halo model has been used for a number of applications, including weak lensing statistics, and relevant for this review, the alignment of galaxies inside dark matter structures on scales from tens of Mpc down to 
tens of kpc. This is possible due to the natural halo model separation into intra- and inter-halo properties, commonly called the 'one-halo' (1h) and 'two-halo' (2h) terms, respectively. Schneider \& Bridle (2010) pioneered the modelling of galaxy alignments in the language of the halo model. As was commonly done for clustering, their formalism explicitly accounted for the distinction between central and satellite galaxies, which is peculiar to intrinsic alignments and effectively doubles the number of contributing terms in the halo model.

Furthermore, as is evident from the equations that follow, the large-scale terms are a rescaling (based on galaxy bias) of the matter clustering, whereas the small scale terms are contributions coming from haloes weighted by their relative abundance. Accordingly, the auto- and cross-power spectra of the galaxy alignments read:

$$
\begin{aligned}
& P_{\mathrm{II}}(k)=P_{\mathrm{II}, \mathrm{cs}}^{1 h}(k)+P_{\mathrm{II}, \mathrm{ss}}^{1 h}(k)+P_{\mathrm{II}, \mathrm{cc}}^{2 h}(k)+P_{\mathrm{II}, \mathrm{cs}}^{2 h}(k)+P_{\mathrm{II}, \mathrm{ss}}^{2 h}(k) ; \\
& P_{\delta \mathrm{I}}(k)=P_{\delta \mathrm{I}, \mathrm{c}}^{1 h}(k)+P_{\delta \mathrm{I}, \mathrm{s}}^{1 h}(k)+P_{\delta \mathrm{I}, \mathrm{c}}^{2 h}(k)+P_{\delta \mathrm{I}, \mathrm{s}}^{2 h}(k),
\end{aligned}
$$

where the subscripts 'c' and 's' stand for central and satellite galaxies, respectively. Schneider \& Bridle (2010) assumed that satellite galaxy ellipticities were uncorrelated with the central galaxy ellipticity. This naturally led to $P_{\mathrm{II}, \mathrm{cs}}^{1 h}(k)=0$. The remaining II one-halo term is:

$$
P_{\mathrm{II}, \mathrm{ss}}^{1 h}(k)=\frac{1}{\bar{n}_{g}^{2}} \int\left\langle N_{g}\left(N_{g}-1\right) \mid M\right\rangle \bar{\gamma}^{2}(M)|w(k \mid M)|^{2} n(M) \mathrm{d} M,
$$

where $\bar{n}_{g}$ is the comoving number density of galaxies, $n(M)$ is the halo mass function (e.g. Press \& Schechter 1974; Bond et al. 1991; Tinker et al. 2008, 2010), and $\left\langle N_{g}\left(N_{g}-1\right) \mid M\right\rangle$ is the second moment of the galaxy number distribution at a given halo mass, $M$. Here, $w(k \mid M)$ is the normalized Fourier transform of the radial 3D-profile of the projected satellite galaxy ellipticities, whereas $\bar{\gamma}(M)$ is the magnitude of the intrinsic shear, $\tilde{\gamma}^{I}$, in a halo of mass $M$.

The model presented by Schneider \& Bridle (2010) also assumed that central galaxies acquired their alignment in the same way their host haloes did. Correspondingly, the $P_{\mathrm{II}, \mathrm{cc}}^{2 h}(k)$ equals the power spectrum in Equation (15). This is a manifestation of the fact that on sufficiently large scales, the dominant term for galaxy alignments is the term that describes the alignment of their host haloes. The term $P_{\mathrm{II}, \mathrm{ss}}^{2 h}(k)$ is formulated by integrating over the joint probability distribution for two haloes of mass $M_{1}$ and $M_{2}$, with $\left\langle N_{g} \mid M_{1}\right\rangle$ and $\left\langle N_{g} \mid M_{2}\right\rangle$ the average number of galaxies in haloes of mass $M_{1}$ and $M_{2}$,

$$
P_{\mathrm{II}, \mathrm{ss}}^{2 h}(k)=\frac{1}{\bar{n}_{g}^{2}} \int\left\langle N_{g} \mid M_{1}\right\rangle \bar{\gamma}\left(M_{1}\right)\left|w\left(k \mid M_{1}\right)\right| n\left(M_{1}\right) \mathrm{d} M_{1} \int\left\langle N_{g} \mid M_{2}\right\rangle \bar{\gamma}\left(M_{2}\right)\left|w\left(k \mid M_{2}\right)\right| n\left(M_{2}\right) \mathrm{d} M_{2} P_{\mathrm{hh}}\left(k \mid M_{1}, M_{2}\right),
$$

where $P_{\mathrm{hh}}\left(k \mid M_{1}, M_{2}\right)=b_{\mathrm{h}}\left(M_{1}\right) b_{\mathrm{h}}\left(M_{2}\right) P_{\delta \delta}^{\text {lin }}(k)$ is the (dark matter) halo-halo power spectrum and $b_{\mathrm{h}}(M)$ is the halo bias function (Tinker et al. 2010). The two-halo central-satellite term is

$$
P_{\mathrm{II}, \mathrm{cs}}^{2 h}(k)=\frac{C_{1} \bar{\rho} a^{2}}{D_{+}} \frac{P_{\delta \delta}^{\operatorname{lin}}(k)}{\bar{n}_{g}} \int\left\langle N_{g} \mid M\right\rangle \bar{\gamma}(M)|w(k \mid M)| b_{h}(M) n(M) \mathrm{d} M .
$$

The terms that define the $P_{\delta \mathrm{I}}(k)$ power spectrum are:

$$
\begin{aligned}
& P_{\delta \mathrm{I}, \mathrm{c}}^{1 h}(k)=0, \\
& P_{\delta \mathrm{I}, \mathrm{s}}^{1 h}(k)=\frac{1}{\bar{n}_{g} \bar{\rho}} \int M\left\langle N_{g} \mid M\right\rangle \bar{\gamma}(M)|w(k \mid M)| u(k \mid M) n(M) \mathrm{d} M, \\
& P_{\delta \mathrm{I}, \mathrm{c}}^{2 h}(k)=-\frac{C_{1} \bar{\rho} a^{2}}{D_{+}} P_{\delta \delta}^{\mathrm{lin}}(k), \\
& P_{\delta \mathrm{I}, \mathrm{s}}^{2 h}(k)=\frac{1}{\bar{n}_{g} \bar{\rho}} \int\left\langle N_{g} \mid M_{1}\right\rangle \bar{\gamma}\left(M_{1}\right)\left|w\left(k \mid M_{1}\right)\right| n\left(M_{1}\right) \mathrm{d} M_{1} \int M\left|u\left(k \mid M_{2}\right)\right| n\left(M_{2}\right) \mathrm{d} M_{2} P_{h h}\left(k \mid M_{1}, M_{2}\right),
\end{aligned}
$$

where $u(k \mid M)$ is the normalized Fourier transform of the radial profile of the matter distribution in a halo of mass $M$ (e.g. Navarro et al. 1997). Note that $P_{\delta \mathrm{I}, \mathrm{c}}^{2 h}(k)=P_{\delta \mathrm{I}}(k)$ from Equation (16) since on sufficiently large scales above around $10 h^{-1} \mathrm{Mpc}$, the central term is the dominant one. 
Schneider \& Bridle (2010) found that the total halo model alignment power spectra are accurately modelled by restricting oneself to the two-halo central correlation on large scales and the one-halo satellite correlation on small scales, i.e.

$$
\begin{aligned}
& P_{\mathrm{II}}(k) \approx P_{\mathrm{II}, \mathrm{ss}}^{1 h}(k)+P_{\mathrm{II}, \mathrm{cc}}^{2 h}(k) \\
& P_{\delta \mathrm{I}}(k) \approx P_{\delta \mathrm{I}, \mathrm{s}}^{1 h}(k)+P_{\delta \mathrm{I}, \mathrm{c}}^{2 h}(k) .
\end{aligned}
$$

The light blue dashed curve in Figure 8 shows the satellite contribution derived from $P_{\delta \mathrm{I}, \mathrm{s}}^{1 h}(k)$, with the free amplitude contained in $\bar{\gamma}$ fitted to the data, along with the other halo model parameters.

\subsection{Transition to intermediate scales}

Alignments on intermediate scales of a few Mpc are difficult to treat, in contrast to alignment models on large scales and alignments inside haloes on small scales. Unifying both regimes would yield a consistent model of alignments over all scales of interest.

Both the linear and quadratic alignment models are derived from (Lagrangian) perturbation theory, as a theory of the tidal interaction of a halo with the gravitational fields generated by the large-scale structure into which the halo is embedded. This naturally implies that the models can only be applied on linear scales above several tens of Mpc. In order to bridge the gap to fully non-linear scales below 1-2 Mpc, where a description according to the halo model would be applicable, it is necessary to modify tidal interactions and take account of the baryonic physics inside the haloes. It is also necessary to have a prescription of clustering and to add observational complications such as redshiftspace distortions due to peculiar motions, where necessary. These intermediate scales, around $2-10 \mathrm{Mpc}$, are referred to as mildly non-linear, and a number of methods have been proposed as phenomenological solutions (Tinker et al. 2005; van den Bosch et al. 2013). Research using simulations (Heymans et al. 2004) and observations (Mandelbaum et al. 2006b) suggest that on these intermediate scales, intrinsic alignments are stronger than predicted by linear theory.

An immediate solution would be to replace the linear matter power spectrum by a non-linear one, which provides stronger alignments on small spatial scales (Bridle \& King 2007). In effect this Non-Linear Alignment (NLA) model asserts that haloes experience stronger tidal fields in non-linearly evolving structures while the linear interaction itself is not changed, leading to increased alignment at non-linear scales.

However, it is possible to imagine that haloes at close separations started interacting directly with each other in a way that would weaken their alignment with the large-scale structure, with the interesting consequence that tidal alignments would be weakened instead of being strengthened in non-linear structures. Nevertheless, the non-linear alignment model is very easy to implement and has proved itself to be more consistent with small-scale observations in comparison to the linear alignment model alone, and thus has remained popular in the literature (Mandelbaum et al. 2011; Kirk et al. 2013; Blazek et al. 2012; Heymans et al. 2013; Chisari et al. 2014; Troxel \& Ishak 2014; Hall \& Taylor 2014).

The non-linear alignment model fails to explain alignments linear in the tidal field on a number of counts, as pointed out by Blazek et al. $(2011,2015)$. While the replacement of the linear dark matter spectrum with a nonlinear one is perfectly suited to describe nonlinearities on small scales, the clustering of galaxies on small scales introduces a non-linear weighting of the ellipticity field by giving rise to many galaxy pairs with small separations. Third, there are non-linear biases both in the physical distribution of galaxies, and to a lesser degree, distortions of the galaxy field due to redshift-space distortions. These effects have an important influence on the shape of the resulting ellipticity correlation function on small scales, most notably on scales $\lesssim 10 \mathrm{Mpc}$, whereas on larger scales the standard linear alignment model is sufficient. It is also common to smooth the tidal field, since fluctuations on scales that are sufficiently small should not have an impact on the observed alignments.

Figure 8 shows the ellipticity-density correlation, $w_{g+}$, for the linear and non-linear alignment (NLA) models and the fit to the one-halo term from Schneider \& Bridle (2010) with observational data and best-fit parameters taken from the Singh et al. (2015) SDSS analysis. It also shows two additional models taken from Blazek et al. (2015), who developed analytic models for galaxy intrinsic alignments based on tidal alignment theory. The black line shows the best-fit standard perturbation theory model that contains non-linear contributions including non-linear clustering, 


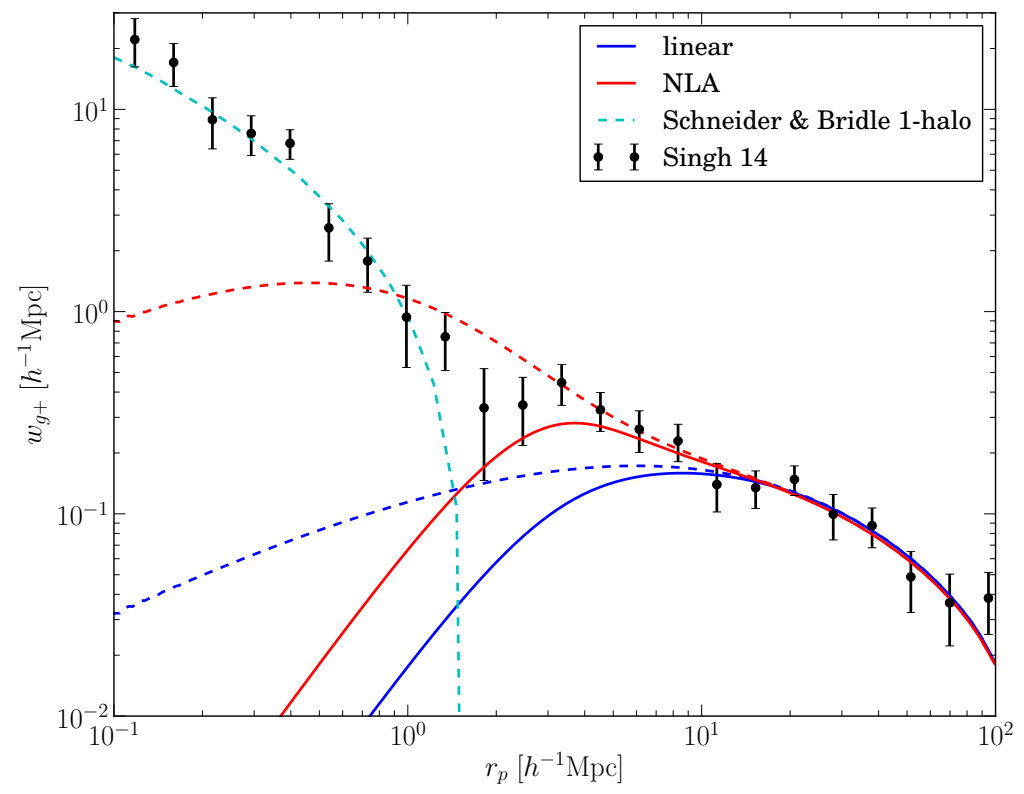

Fig. 8.-: Galaxy position ellipticity correlations found in SDSS luminous red galaxies from Singh et al. (2015) in comparison to models based on linear alignments (dark blue line), enhanced by non-linear structures (NLA; red line), and including non-linear clustering effects, biasing and galaxy density weighting (black line), with Gaussian smoothing at $k=1 h^{-1} \mathrm{Mpc}$ (solid lines) and without smoothing (dashed lines). The light blue dashed line is the fit to the one-halo (1-halo) term from Schneider \& Bridle (2010) and the green line shows a non-perturbative NFW-based one-halo model with linear theory for the two-halo term. The black and green models are reproduced from Blazek et al. (2015). Figure credit: Jonathan Blazek E Sukhdeep Singh.

halo bias and galaxy density weighting. The green line shows a physically motivated and non-perturbative one-halo model based on the halo profile of Navarro-Frenk-White ( $\mathrm{NFW}^{9}$; see Navarro et al. 1996) combined with linear theory for the two-halo term, including a contribution that captures the enhancement of the tidal field in locations where galaxies form. The small wiggles in the green line at small scales are due to the use of observed galaxy clustering, since the model makes a prediction for the alignment per galaxy, rather than the total $w_{g+}$ signal. The figure also shows the effects of smoothing of the tidal field on the linear and non-linear alignment models, where the dashed lines show the models with no smoothing, while the solid lines show the models with smoothing with a Gaussian of width $k=1 h^{-1} \mathrm{Mpc}$. Motivated by observational results, the NFW-based model includes saturation of the alignment (i.e. the alignment signal does not increase any further) per galaxy within the halo virial radius. The linear alignment model matches the observations well on scales $\gtrsim 10 h^{-1} \mathrm{Mpc}$ and the one-halo fit matches the observations on scales $\lesssim 1 h^{-1} \mathrm{Mpc}$, while the non-linear alignment model, both with and without smoothing, provides a reasonable approximation to the intermediate scales. However, the models from Blazek et al. (2015), which include additional non-linear effects, show a much closer match to the data, with the NFW-based one-halo plus linear two-halo model effectively unifying the small, intermediate and large scales.

The alignments of spiral galaxies in non-linear environments are also affected on small scales by galaxy clustering and redshift-space distortions due to peculiar motion, which are partially already included in current analytical models. Because the primary angular momentum build-up of haloes takes place at very high redshifts it might be assumed that

${ }^{9}$ Navarro et al. (1996) fitted a universal density profile to dark matter haloes in $N$-body simulations. This profile is now known as the NFW profile. 
the angular momentum direction is conserved, since secondary effects, like the shaping of the ellipticity correlation function due to peculiar motion of galaxies, or the lensing mapping of intrinsically shape-correlated galaxies, has been shown to be small (Giahi \& Schäfer 2013; Giahi-Saravani \& Schäfer 2014).

However, results from numerical simulations are not conclusive on this issue. Some dark matter simulations confirm this picture (e.g. Porciani et al. 2002), but recent simulations including baryons found spin changes due to the merging of subhaloes (e.g. Dubois et al. 2014; Cen 2014). It seems that ultimately the properties of the galactic disc and its orientation due to an initial tidal interaction and subsequent merging and accretion is very difficult to predict (Dekel et al. 2009; Hahn et al. 2010; Danovich et al. 2015), which could potentially be solved by numerical simulations (see Sections 4.2.2, 5.1.1 and 5.2.1).

\subsection{Theory and Modelling Roundup}

In this section the established alignment models for galaxies on large scales have been reviewed, starting from the idealising theory of tidal interactions, which give rise to the linear and quadratic alignment models, that are thought to govern the alignment of spiral galaxies through the angular momentum generation, and that of elliptical galaxies through tidal stretching on large scales. The halo model was also reviewed, for an effective description of alignment on small scales. The transition between the two regimes nicely illustrates complications in constructing alignment models, which to some extent are not present on larger scales or are encapsulated in an effective description on smaller scales. These include mildly nonlinear structure formation, which is in principle accessible by perturbation theory, and effects which shape intrinsic correlations such as clustering and peculiar motion effects, up to alignment with nonlinear structures on small scales.

These issues, as well as the idealising assumptions for the tidal interactions themselves, suggest that improvement can be expected by numerical simulations of structure formation, which are able to address nonlinear structure formation on small scales as well as halo formation, and depending on the simulation, other effects that have an influence on the shape and amplitude of shape correlations on small scales.

Specifically, simulations fall into two categories: $N$-body simulations (see Section 4), where only the collisionless dark matter component is simulated, aim at the statistics of the tidal fields and the orientation of dark matter haloes in those fields, ultimately down to scales where direct interaction between haloes and the dynamics of tidal interaction plays a role. Contrarily, hydrodynamic simulations (see Section 5) answer questions related to the shape of the luminous component of galaxies in relation to the host halo properties and the influence of baryonic components onto the shape of a galaxy.

\section{4. $N$-Body simulations}

On the largest scales, the evolution of the Universe can be investigated analytically and with Gaussian random fields. However, on smaller scales, the Universe can be distinctly non-linear (structures would not collapse without these non-linear effects) and it is not possible to probe the formation and evolution of non-linear structures analytically, which is why an alternative approach had to be found. $N$-body simulations mimic the statistical properties of the Universe by sampling the dark matter density field with discrete particles. These particles are placed in a box and since it is not computationally possible to represent every atom in the Universe with its own particle, each particle represents a sampling of the volume at that particular point in space. The particles are generally very massive, adding together to make the total density of the volume equal to the average density of the Universe. For example, a high resolution, cosmological volume ( $\left.\gtrsim 100 h^{-1} \mathrm{Mpc}\right)$ simulation today might have a particle mass of $\sim 10^{6} h^{-1} \mathrm{M}_{\odot}$. This distribution of particles is imprinted with a theoretical power spectrum of fluctuations from when the Universe was still in a linear state. As the simulation moves forward through time, the dark matter particles act under gravity and evolve over cosmological time. With an appropriate choice of initial condition power spectrum and subsequent dark matter evolution, cosmological $N$-body simulations are able to produce particle distributions that resemble the statistical distribution of matter in our Universe today.

$\mathrm{N}$-body simulations are used for alignment studies because they provide a data set with known parameters, which is essential when trying to understand the alignment signals. They can also provide a statistical sample - large numbers 
of individual dark matter haloes can be identified in a single realization. There are many different measurements made in order to quantify the alignment signal and sometimes many different ways of measuring the same property, and these allow for robustness tests of the results (see Section 4.1). Most importantly, $N$-body simulations are able to provide data on how the shapes, spins and angular momenta of dark matter haloes align over a wide range of scales and in different environments (see Section 4.2).

Another possibility is to investigate a smaller volume simulation with a much higher resolution. This is known as a 'zoom' simulation, where a region of interest is selected within a larger-scale simulation and then resimulated with higher resolution. The advantages of this technique are that the large-scale tidal forces are still present to influence the region of interest, but the higher resolution allows a much more detailed study to be performed.

$N$-body simulations alone can not provide a complete picture of the formation and evolution of intrinsic galaxy alignments. The most important limitation is that they do not contain any galaxies. It is also now known that baryons have an impact on the shapes, orientations, spins and angular momenta of dark matter haloes. Investigations of these effects must be studied using hydrodynamic simulations (see Section 5). Despite their limitations, $N$-body simulations will remain an important tool in the understanding of intrinsic alignments. They are much faster to run than their hydrodynamic counterparts and the dark matter physics is well understood (unlike baryon physics). Most importantly, they provide the backbone for the semi-analytic modelling of galaxy properties (see Section 6), which will likely become the standard data sets for the galaxy intrinsic alignment investigations of the future.

\subsection{What do people measure?}

There are a number of things that must be determined in order to measure the alignments of dark matter haloes in $N$-body simulations. The first step is to identify the haloes within the simulation (Section 4.1.1). It is then possible to determine the halo shapes (Section 4.1.2) and the angular momentum or spin of the halo (Section 4.1.3). Some studies investigate alignments with respect to the environment that the halo resides in, which requires the simulation to be classified into cosmic web elements (Section 4.1.4). With this information in hand, it is possible to measure the alignments of the dark matter haloes (Section 4.1.5). Table 1 provides a list of possible alignments, indicating where individual alignments are discussed in more detail either in this review or if they can only be found in (Kirk et al. 2015). While this section is focused on $N$-body simulations, these measurements outlined below are largely the same for hydrodynamic simulations (see Section 5), with the shape of the stellar and/or gas component also being measured.

\subsubsection{Dark matter haloes}

In order to study the shapes and alignments of dark matter halos in $N$-body simulations, it is useful to provide a formal definition of a halo and some typical methods used to identify them. From a theoretical perspective, a halo is defined as an object whose constituent particles are gravitationally bound. However, this can be difficult to define in practice, given that simulations are discretely sampled by particles, which trace the local density and gravitational potential.

A simple model for the formation of haloes is the spherical collapse model (e.g. Press \& Schechter 1974), which describes the formation of a collapsed object by considering the evolution of a sphere of uniform overdensity, $\delta$, in a smooth background. The overdensity initially expands with the expansion of the Universe; however, as it is overdense, the expansion slows, and eventually begins to collapse under its own self-gravity. The overdensity required for a halo to collapse is related to the underlying matter density. After a period of relaxation, the halo ultimately reaches virial equilibrium. For a spatially flat universe today, the overdensity of a fully collapsed, virialised halo (relative to the critical density) is given by ${ }^{10} \Delta_{c} \simeq 178$, which is often rounded up to $\Delta_{c}=200$ for simplicity in simulation analyses. It is important to note that the value for $\Delta_{c}$ is dependent on the cosmology and redshift of the universe (at earlier times $\Delta_{c}$ will be a smaller number). In $N$-body simulations, and indeed in weak lensing mass measurements, the virial mass of a halo, $M_{v i r}=M_{\Delta_{c}}$, is typically computed within the virial radius, $R_{\mathrm{vir}}=R_{\Delta_{c}}$, which is the radius at which the density of the halo is $\Delta_{c}$ times the critical density of the universe.

\footnotetext{
${ }^{10}$ Note that some studies consider overdensities relative to the underlying matter density, rather than the critical density. Such overdensities can be easily scaled, due to the fact that $\Omega_{M}$ is the ratio between the mean matter density and the critical density.
} 


\begin{tabular}{|c|c|c|c|c|c|}
\hline Shape of & Aligned with & Symbol & Figure(s) & Equation(s) & Section(s) or Paper \\
\hline Halo & Shape of itself at different radius & $\overline{\theta_{\mathrm{H}}\left(\mathrm{R}_{1}, \mathrm{R}_{2}\right)}$ & $2,9,10,15$ & (4) & Sections 4.2 .2 and 5.1.1 \\
\hline Halo & Halo Shape & $\Theta_{\mathrm{HH}}$ & $3 b$ & $(4),(47)$ & Section 4.2 .4 \\
\hline Halo & Halo Position & $\Theta_{\mathrm{Hh}}$ & $3 b$ & $(4),(46)$ & Sections 4.2 .4 and 4.2 .5 \\
\hline Halo & Sheet/Wall Shape & $\theta_{\mathrm{HW}}$ & $4 \mathrm{a}$ & (4) & Section 4.2 .5 \\
\hline Halo & Void Position of Centre & $\theta_{\mathrm{Hv}}$ & $4 \mathrm{a}$ & (4) & Section 4.2 .5 \\
\hline Halo & Filament Shape & $\theta_{\mathrm{HF}}$ & $4 \mathrm{~b}$ & (4) & Section 4.2 .5 \\
\hline Central & Halo Shape & $\theta_{\mathrm{CH}}$ & $3 b, 13$ & (4) & Sections 5.1.1, 5.2.2 and 6 \\
\hline Central & Central Shape & $\Theta_{\mathrm{CC}}$ & $3 b$ & (4) & Kirk et al. (2015) \\
\hline Central & Central Position & $\Theta_{\mathrm{Cc}}$ & $3 b$ & (4) & Section 5.2 \\
\hline Central & Satellite Distribution Shape & $\Theta_{\mathrm{CB}}$ & $3 b$ & (4) & Section 5.2 .2 \\
\hline Satellite & Halo Position of Centre & $\theta_{\mathrm{Sh}}$ & $3 a, 12$ & (4) & Sections 4.2 .3 and 5.1.2 \\
\hline Satellite & Halo Shape & $\theta_{\mathrm{SH}}$ & $3 a^{*}$ & (4) & Sections 4.2 .4 and 4.2 .5 \\
\hline Satellite & Central Position & $\theta_{\mathrm{Sc}}$ & $3 a^{*}$ & (4) & Kirk et al. (2015) \\
\hline Satellite & Central Shape & $\theta_{\mathrm{CS}}$ & $3 a$ & (4) & Kirk et al. (2015) \\
\hline Satellite & Satellite Shape & $\theta_{\mathrm{SS}}$ & $3 a$ & $(4)$ & Kirk et al. (2015) \\
\hline Satellite Distribution & Halo Shape & $\theta_{\mathrm{BH}}$ & $3 a$ & (4) & Sections 4.2 .3 and 5.1.2 \\
\hline Satellite Distribution & Central Shape & $\theta_{\mathrm{BC}}$ & $3 a$ & (4) & Sections 4.2 .3 and 5.1.2 \\
\hline Satellite Distribution & Satellite Distribution Shape & $\Theta_{\mathrm{BB}}$ & $3 b^{*}$ & (4) & Kirk et al. (2015) \\
\hline Satellite Distribution & Satellite Distribution Position & $\Theta_{\mathrm{Bb}}$ & $3 b^{*}$ & (4) & Kirk et al. (2015) \\
\hline Galaxy & Halo Shape & $\theta_{\mathrm{GH}}$ & $3 a, 14$ & (4) & Section 5.2.2 \\
\hline Galaxy & Wall Shape & $\theta_{\mathrm{GW}}$ & $4 a^{*}$ & (4) & Section 5.1.3 \\
\hline Galaxy & Void Position & $\theta_{\mathrm{Gv}}$ & $4 a^{*}$ & (4) & Kirk et al. (2015) \\
\hline Galaxy & Filament Shape & $\theta_{\mathrm{GF}}$ & $4 b^{*}$ & $(4)$ & Section 5.1.3 \\
\hline
\end{tabular}

Table 1:: Intrinsic alignment observable misalignment angles within one halo, $\theta$, and across two haloes, $\Theta$, for shape (upper case letter) and position (lower case letter) of dark matter haloes $(\mathrm{H})$, central galaxies (C) satellites (or dark matter subhaloes; S) the shape of the satellite distribution (which is defined by the satellite positions; B), a complete galaxy sample (includes centrals and satellites; G), walls (W), filaments (F) and the position of the centre of a void (v). The first and second of these indices denote the object in the first and second columns of the table respectively. Note that not all of the alignments listed above are shown in Figures 2 to 4 . However, if the alignment listed is not explicitly shown, the figure is denoted with $\mathrm{a}^{*}$ and it should be straightforward to infer the alignment from the named figure.

The simplest method to identify particles that have coalesced into a collapsed halo is to simply group particles together based on their proximity to neighbouring particles. Such a scheme is known as "Friends-of-Friends" (FOF) (e.g. Davis et al. 1985). A typical FOF halo finder will identify all particles that are separated by less than a userspecified linking length, and will group these together as a halo. This type of algorithm is relatively straightforward to implement, and can be run during the evolution of an $N$-body simulation, allowing for a simple way to trace the evolution of structures in such simulations. However, the mass associated with a FOF halo, computed by simply summing the masses of the particles identified as belonging to the halo, is strongly dependent on the choice of linking length, and it is not trivial to relate this mass to something more physically motivated, such as the virial mass.

An alternative approach to halo finding is the "Spherical Overdensity" (SO) method (Lacey \& Cole 1994). The basic principle involves identifying local density maxima, and identifying a spherical region around each maximum, within which the mean overdensity is above some pre-defined level. The link between this, and the spherical collapse model, should be immediately evident. Many different variants of this general scheme have been adopted in the literature, such as the "Bound Density Maximum" (BDM) technique (e.g. Klypin \& Holtzman 1997). In contrast to FOF halos, those identified with SO or BDM techniques can overlap, and it is therefore straightforward with these latter methods to identify subhaloes within larger structures (i.e. halos that lie entirely within the virial radius of a larger structure).

Most commonly used halo finders use modifications to, or combinations of, the above methods; reviews of contem- 
porary methods can be found in Bett et al. (2007) and Knebe et al. (2011).

\subsubsection{Dark matter halo shapes}

Once the dark matter haloes have been identified, their shapes (halo axes) must be identified in order to measure any shape alignments. The shapes of these haloes can be determined by considering the distribution of their constituent particles. Dark matter haloes are typically modelled as being ellipsoidal, and although this is a simplification of the more complex $N$-body halo shape, this appears to work very well. One common approach to measuring shapes uses the so-called inertia tensor ${ }^{11}$ for the dark matter particles that have been identified as being a part of the halo (see Section 4.1.1),

$$
I_{i j}=\frac{\sum_{n} w_{n} m_{n} x_{n i} x_{n j}}{\sum_{n} w_{n} m_{n}}
$$

where $m_{n}$ represents the mass of the $n^{\text {th }}$ particle, $w_{n}$ represents a radial weight function to be discussed shortly, and $x_{n i}, x_{n j}$ represent the position coordinates of the $n^{\text {th }}$ particle with respect to the halo centre (where the centre is the location of the gravitational potential minimum), with $0 \leq i, j \leq 2$ for 3D and $0 \leq i, j \leq 1$ for 2D. The eigenvectors, $\boldsymbol{e}_{1}, \boldsymbol{e}_{2}, \boldsymbol{e}_{3}$, of this tensor represent the principal axes of the ellipsoids with the lengths of the principal axes given by the square roots of the eigenvalues $\lambda_{i}$, such that $\sqrt{\lambda_{1}} \geq \sqrt{\lambda_{2}} \geq \sqrt{\lambda_{3}}$.

The use of $w_{n}=1$ results in the so-called unweighted inertia tensor, whereas $w_{n}=1 / d_{n}^{2}$ (where $d_{n}$ is the weighted distance relative to the centre of the halo) is the reduced inertia tensor, which gives more weight to the centres of haloes. The reduced inertia tensor can be computed using a spherically symmetric weight function proportional to the inverse distance of the particle from the halo centre, i.e. defining $d_{n}^{2}=x_{n}^{2}+y_{n}^{2}+z_{n}^{2}$; or, alternatively, using an ellipsoidal weighting function that defines $d_{n}^{2}=x_{n}^{2}+\left(y_{n} / q\right)^{2}+\left(z_{n} / s\right)^{2}$, where $q=b / a$ and $s=c / a$, and $a \geq b \geq c$ are the semi-major, intermediate and semi-minor axes of the halo. When using the ellipsoidal weighting function, the inertia tensor is typically solved for iteratively.

When measuring the shapes of dark matter haloes, it is important to consider the number of particles being used to make the shape measurement. Bett et al. (2007) performed resolution tests on their 3D shape measurements and showed that shapes were biased toward being less spherical (more prolate) when the number of particles in the halo was $\lesssim 300$. Similarly, Jing (2002) found that their 2 D projected shape measurements converged with a minimum of 160 particles. It was not uncommon for some early studies mentioned in this review to include haloes with as few as 10 or 20 particles, which offers a reasonable explanation for why some early results may be in contrast to more recent conclusions. Given these known biases, it is remarkable how many early works found results actually in agreement with the current consensus (see Section 4.2).

An alternative shape measurement approach was suggested by Jing \& Suto (2002), who noted that while the iterative approach detailed above worked well for low-resolution simulations, it does not converge consistently in the case of high resolution data due to the iterative approach being unstable in the presence of significant substructures (which are more readily resolved in the high-resolution simulations). The alternative approach calculates isodensity surfaces at different overdensities to determine the axes of the halo. To start, the local density is calculated at the position of each particle. A spherically symmetric spline kernel, often used in smoothed particle hydrodynamics calculations (SPH; e.g. Hernquist \& Katz 1989; Monaghan \& Lattanzio 1985), is adopted,

$$
\mathcal{W}\left(r_{s}, h_{i}\right)=\frac{1}{\pi h_{i}^{3}}= \begin{cases}1-\frac{3}{2}\left(\frac{r_{s}}{h_{i}}\right)^{2}+\frac{3}{4}\left(\frac{r_{s}}{h_{i}}\right)^{3} & \left(r_{s} \leq h_{i}\right) \\ \frac{1}{4}\left(2-\frac{r_{s}}{h_{i}}\right)^{3} & \left(h_{i}<r_{s}<2 h_{i}\right) \\ 0 & \text { otherwise, }\end{cases}
$$

where $h_{i}$ is the smoothing length of the $i$ th particle. The local density of a particle, $\rho_{i}$ is computed by taking the 32 nearest neighbour particles, where $r_{s}$ is distance of the neighbour particle from the $i$ th particle, and applying their

${ }^{11}$ This equation is actually the quadrupole tensor of the mass distribution, but as it is regularly referred to as the moment of inertia tensor in the literature, this is the convention followed throughout this review. 
contribution to the local density using the weighting in Equation (34). The smoothing length of the particle, $h_{i}$, is often set to be half the radius $r_{s}$. After the local density of the particles has been calculated, isodensity surfaces can be determined at different overdensities, which correspond to different halo radii, and a triaxial fit can be used to determine the axes.

Another alternative approach to measuring the shape of a dark matter halo is to determine the shape of the potential of the halo (e.g. Kuhlen et al. 2007). The unweighted potential energy tensor, $W_{i j}=\sum_{n} x_{n i} \mathrm{~d} \Phi / \mathrm{d} x_{n j}$, is related to the (unweighted) kinetic energy tensor,

$$
K_{i j}=\frac{1}{2} \sum_{n} m v_{n i} v_{n j},
$$

where $v$ is the velocity of the particle, through the tensor virial theorem,

$$
\frac{1}{2} \frac{d^{2} I_{i j}}{d t^{2}}=2 K_{i j}+W_{i j},
$$

where $t$ is time. For a relaxed dark matter halo, the term on the left of this equation should be zero at $z=0$. In this case, the eigenvectors of the diagonalised unweighted kinetic energy tensor (Equation 35) will provide the principal axes of the potential ellipsoid.

The axes derived using any of the shape measurement methods above can also be used to measure the triaxiality of the halo.

$$
\mathcal{T}=\frac{\left(a^{2}-b^{2}\right)}{\left(a^{2}-c^{2}\right)}=\frac{\left(1-q^{2}\right)}{\left(1-s^{2}\right)}
$$

The axis ratio $s$ is typically referred to as the sphericity of the halo. In general, a halo is oblate when $\mathcal{T}<\frac{1}{3}$, prolate when $\mathcal{T}>\frac{2}{3}$ and triaxial otherwise.

\subsubsection{Angular momentum and spin}

Tidal torque theory presents a framework for how angular momentum is built up in galaxies and dark matter haloes (see Section 3.1.1). Initial angular momentum predictions can be made analytically (e.g. Schäfer 2009), while structure formation is in the linear regime. At the time of halo turnaround, when a halo begins to collapse and the processes acting on it become non-linear, tidal torquing leaves an imprint of the tidal field in which the halo formed. This is the point where analytic models break down and the continued build up of angular momentum, through processes including mergers and accretion, is best traced through simulations (both $N$-body and hydrodynamic). The imprint of the early tidal field is expected to remain present through these processes, as has been shown using $N$-body simulations in Porciani et al. (2002). The structures in the cosmic web are physical manifestations of the tidal field and these cosmic web elements have reasonably uniform and symmetric morphologies (filaments in particular), which on large-scales present a uniform tidal field (i.e. haloes within a filament would all experience similar tidal forces; Trowland et al. 2013). As a result, the angular momentum vectors of neighbouring haloes within cosmic web elements should show some alignments at small separations. Moreover, the angular momentum of the haloes should also align in the direction of the overdensities in the large-scale-structure (e.g. along the semi-major axis of a filament or in the plane of a sheet). However, there are a number of influences that can cause these alignments to change over time and this is discussed further in Section 4.2.2.

Similar to the shape measurements, the number of particles in a halo has an affect on the measurement of the angular momentum and spin. Bett et al. (2007) performed resolution tests on their spin measurements and found that the median spin parameter was biased high when the number of particles in the halo was $\$ 300$. Caution should also be exercised on results from the spin/angular momentum literature that use fewer than 300 particles per halo.

The angular momentum of an $N$-body dark matter halo can be computed as:

$$
\boldsymbol{J}=\sum_{n} m_{n} \boldsymbol{R}_{n} \times \boldsymbol{v}_{n}
$$


where the sum is over the total number of particles, $N$, identified within a particular halo, $\boldsymbol{R}_{n}$ is the radial distance of the $n^{\text {th }}$ particle relative to the halo centre and $\boldsymbol{v}_{n}$ is the velocity of the $n^{\text {th }}$ particle relative to the halo centre. Similarly, one can compute the specific angular momentum

$$
\boldsymbol{j}=\frac{1}{N} \sum_{n} \boldsymbol{R}_{n} \times \boldsymbol{v}_{n},
$$

and the cumulative specific angular momentum profile within the halo radius $R$,

$$
\boldsymbol{j}(\leq R)=\frac{1}{M(\leq R)} \sum_{\leq R} m_{n} \boldsymbol{R}_{n} \times \boldsymbol{v}_{n}
$$

The spin parameter is a dimensionless measure of the amount of rotation in a dark matter halo. Under the standard definition from Peebles (1969), the spin parameter is given by

$$
\lambda=\frac{J|E|^{1 / 2}}{G M^{5 / 2}},
$$

where $J$ is the magnitude of the angular momentum $J, E$ is the total energy of the halo, and $M$ is the halo mass. Due to the difficulty in measuring the total halo energy, Bullock et al. (2001) introduced a modified spin parameter

$$
\lambda^{\prime}=\frac{J}{\sqrt{2} M V R},
$$

where $V$ is the circular velocity at halo radius $R$ such that $V^{2}=G M / R$.

\subsubsection{Cosmic web elements}

Numerical simulations and large-scale redshift surveys have provided a picture of the large-scale structure of the Universe. This cosmic web is comprised of clusters, filaments, sheets/walls and voids. Topologically, filaments are expected join with clusters and border sheets, which are expected to reside at the boundary of the void surfaces. The method to recover the topology of the large-scale density field must account for the density field being discretely sampled in simulations (and indeed in observations as well), while still providing a consistent classification of web elements.

A number of algorithms exist to define the cosmic web elements in simulations, and three of these are based on the same basic principle. For each, a locally symmetric tensor is diagonalised to give three real eigenvalues, $\lambda_{1}>\lambda_{2}>\lambda_{3}$ with corresponding eigenvectors $\hat{e}_{1}, \hat{e}_{2}$ and $\hat{e}_{3}$. Classification into the four different cosmic web types, void, sheet, filament and cluster, is related to the number of eigenvalues above a given threshold $\lambda_{t h}$, where a common choice is $\lambda_{t h}=0$ (e.g. Hahn et al. 2007a,b; Codis et al. 2012; Trowland et al. 2013). The value of $\lambda_{t h}$ is not always zero and some studies set its value through visual inspection of the simulated cosmic web, as this is thought to produce a cosmic web that more accurately matches observational evidence (e.g. Libeskind et al. 2012, 2013).

This basic procedure to define web elements is performed on the tidal shear tensor (ignoring the factor of $4 \pi G$, see Section 3.1.1; e.g. Hahn et al. 2007a,b; Codis et al. 2012; Forero-Romero et al. 2014),

$$
T_{i j}=\frac{\partial^{2} \Phi}{\partial x_{i} \partial x_{j}},
$$

the velocity shear tensor, where the Hubble constant $H_{0}=100 h \mathrm{~km} \mathrm{~s}^{-1} \mathrm{Mpc}^{-1}$ (e.g. Libeskind et al. 2013; ForeroRomero et al. 2014),

$$
\Sigma_{i j}=\frac{1}{2 H_{0}}\left(\frac{\partial v_{i}}{\partial x_{j}}+\frac{\partial v_{j}}{\partial x_{i}}\right)
$$


and the field defined by the Hessian of the smoothed density field, $\mathcal{S}(\rho)$ (e.g. Aragón-Calvo et al. 2007; Zhang et al. 2009; Trowland et al. 2013),

$$
\mathcal{H}_{i j}=\frac{\partial^{2} \mathcal{S}(\rho)}{\partial x_{i} \partial x_{j}} .
$$

For both $T_{i j}$ and $\Sigma_{i j}$, the classification is such that zero, one, two or three eigenvalues above $\lambda_{t h}$ corresponds to void, sheet, filament and cluster respectively. However for $\mathcal{H}_{i j}$, the sign of the eigenvalues is opposite. This is due to the potential calculated in $T_{i j}$ being derived from the matter density distribution through the Poisson equation, making $\Phi$ a Fourier transform of the density field. Thus, zero, one, two or three eigenvalues above $\lambda_{t h}$ corresponds to cluster, filament, sheet and void respectively.

While the basic premise of these classification schemes remains consistent within the literature, the details of the implementations differ and many works include extensions to the methods to form more robust classifications. The reader is advised to consult the references directly for the fine details of each scheme. Additionally, many alternative classification schemes exist - too many to mention them all here. For a comprehensive list of alternative schemes and the development of the field, see Sousbie et al. (2009) and references therein.

\subsubsection{Alignments}

The simplest method to measure alignments is to determine a misalignment angle, $\theta$ (or $\Theta$ between two haloes), between the axes (semi-major, intermediate, semi-minor or spin/angular momentum) of the halo (or haloes) such that $\cos (\theta)=1$ is a parallel alignment and $\cos (\theta)=0$ is perpendicular alignment.

When considering the alignments between two haloes in three-dimensions, it is common to give each halo a $3 \mathrm{D}$ orientation unit vector $\hat{l}$. Again, this orientation can be associated with the direction of any of the axes of the halo or the direction of the angular momentum/spin vector. The 3D unit vector in the direction connecting the two haloes is defined as $\hat{\boldsymbol{r}}$. With these definitions, it is possible to determine whether neighbouring halo axes tend to point in the same direction,

$$
\mathcal{A}_{l l}(r)=\left\langle\left|\hat{\boldsymbol{l}}_{i} \cdot \hat{\boldsymbol{l}}_{j}\right|\right\rangle,
$$

and if haloes tend to point in the direction of their neighbours,

$$
\mathcal{D}_{l r}(r)=\langle|\hat{\boldsymbol{l}} \cdot \hat{\boldsymbol{r}}|\rangle \equiv \frac{1}{N} \sum_{i, j}\left|\hat{\boldsymbol{l}}_{i} \cdot \hat{\boldsymbol{r}}_{i j}\right| .
$$

Both of these correlations correspond to $\langle|\cos (\Theta)|\rangle$, where $\Theta$ is the misalignment angle between the two halo axes in Equation (46) or between the halo axis and the vector connecting the two haloes in Equation (47) (e.g. Faltenbacher et al. 2002; Bailin \& Steinmetz 2005; Kasun \& Evrard 2005). An alternative is to investigate the square of the dot products in Equations (46) and (47), since the halo axes have no preferred direction (i.e. $\pm \cos (\Theta)$ are physically identical). This is equivalent to measuring $\left\langle\left|\cos ^{2}(\Theta)\right|\right\rangle$ (e.g. Hopkins et al. 2005).

It is also common to measure the shape of haloes as viewed in projection on the plane of the sky to match observations. The projected shape of a halo is typically defined by the moment of inertia tensor (Equation 33 and surrounding text), in a method that is analogous to calculations of galaxy surface brightness distributions (e.g. Miralda-Escude 1991). An ellipticity can then be defined as $\epsilon=\epsilon_{1}+i \epsilon_{2}$, with components given by

$$
\begin{gathered}
\epsilon_{1}=\frac{I_{11}-I_{22}}{I_{11}+I_{22}} \\
\epsilon_{2}=\frac{2 I_{12}}{I_{11}+I_{22}},
\end{gathered}
$$

such that

$$
\epsilon_{i}=\frac{1-q^{2}}{1+q^{2}}\{\cos (2 \varphi), \sin (2 \varphi)\},
$$


where $q$ is the axis ratio and $\varphi$ is the position angle of the halo. From these ellipticity measurements, an ellipticity correlation function can be constructed that is analogous to Equation (46) (see also Equation 4),

$$
\xi_{\epsilon \epsilon}(r)=\left\langle\left|\epsilon_{i}(\boldsymbol{x}) \cdot \epsilon_{j}(\boldsymbol{x}+\boldsymbol{r})\right|\right\rangle \equiv\left\langle\epsilon^{S} \epsilon^{\prime S}\right\rangle,
$$

computed over all pairs of haloes with a 3D separation vector connecting the two haloes defined as $\boldsymbol{r}$ (e.g. Croft \& Metzler 2000; Heavens et al. 2000; Jing 2002).

Comparing with the correlations in Equation (8), the alignments in Equations (46) and (50) are equivalent to the II term and the shape-density alignments in Equation (47) are equivalent to the $\delta \mathrm{I}$ term, which gives rise to the GI correlations when also accounting for gravitational shear of the luminous galaxies.

\subsection{Results}

In this section, the results from the $N$-body literature are presented by alignment type. Section 4.2.1 starts with the general shapes and spins of dark matter haloes. The section then treats alignments as a function of their scale, starting with dark matter halo internal alignments in Section 4.2.2 and moving to halo-satellite alignments in Section 4.2.3, then halo-halo alignments in Section 4.2.4 before finally presenting dark matter halo alignments with the large-scale structure in Section 4.2.5.

\subsubsection{Halo shapes and spins}

There is consensus among many $N$-body simulation studies that dark matter haloes tend to be strongly aspherical, with a preference for prolate over oblate haloes (e.g. Davis et al. 1985; Frenk et al. 1988; Warren et al. 1992; Kasun \& Evrard 2005; Shaw et al. 2006; Allgood et al. 2006; Schneider et al. 2012). When considering a full sample of haloes, independent of environment, haloes tend to become more prolate with increasing mass (e.g. Kasun \& Evrard 2005; Paz et al. 2006; Hahn et al. 2007b; Schneider et al. 2012). Dark matter haloes also tend to collapse to a shape that is more triaxial and become more spherical over time (Hopkins et al. 2005). There appears to be no significant dependence of shape parameters on environment for massive haloes $\left(M_{v i r}>2 \times 10^{12} h^{-1} \mathrm{M}_{\odot}\right)$, although environment does play a significant role for less massive haloes in clusters, which tend to be less spherical and more prolate, while haloes in filaments tend to be more oblate (Hahn et al. 2007a). In addition, there is some evidence to suggest that haloes that reside close to clusters and haloes with little substructure are more spherical (Ragone-Figueroa \& Plionis 2007). Virialised (relaxed) haloes are more spherical than haloes that have experienced a lot of mergers and contain substructure (which causes them to be more elongated) and haloes in high density environments like clusters will have formed earlier and had more time to relax, resulting in their more spherical nature (Ragone-Figueroa \& Plionis 2007).

The distribution of halo spin parameters tends to be described as a lognormal distribution (e.g. Davis et al. 1985; Warren et al. 1992; Cole \& Lacey 1996; Bailin \& Steinmetz 2005; Shaw et al. 2006; Davis \& Natarajan 2009),

$$
p(\log \lambda)=\frac{1}{\lambda \sqrt{2 \pi} \sigma} \exp \left[-\frac{\log ^{2}\left(\lambda / \lambda_{0}\right)}{2 \sigma^{2}}\right],
$$

where $\lambda_{0}$ is the median spin parameter (the location of the peak), and $\sigma$ is the scatter in $\log \lambda$ (Davis et al. 1985; Bullock et al. 2001; Maller et al. 2002; Bailin \& Steinmetz 2005; Avila-Reese et al. 2005). This distribution tends to be rather insensitive to the choice of cosmological parameters, and remains essentially unchanged whether the spin parameter includes all the mass out to the virial radius, or only the mass within a truncated radius e.g. $0.12 R_{\text {vir }}$ (Bailin et al. 2005). The log-normal distribution of spins can be modelled equally well by linear tidal-torque theory applied to shells of collapsing material or with a model based on the transfer of the orbital angular momentum of merging satellites to the internal spin of the halo (Maller et al. 2002). Furthermore, Vitvitska et al. (2002) found that a random walk model to build up angular momentum naturally produced a lognormal distribution of the spin parameter, though they only tracked the major progenitor of each halo during its evolution, rather than all the mass in the halo. Hahn et al. (2007a) showed that this lognormal model did not fit the distribution for spin parameters of $\lambda>0.1$ in their simulations, while Bett et al. (2007) found the lognormal distribution to be generally a poor fit to the spin distribution of the $>10^{6}$ haloes in their catalogue. They suggested that while this fit was sufficient for low numbers of objects, it avoided the very low spin values that were present in the catalogue. 


\subsubsection{Internal alignments}

Central galaxies form in the inner region of a dark matter halo. It is therefore instructive to consider the shape and orientation of the halo at the radius of interest to the galaxy as well as a range of larger radii (and not simply at the virial radius).

The shape of a dark matter halo changes with radius (e.g. Frenk et al. 1988; Dubinski \& Carlberg 1991; Warren et al. 1992; Jing \& Suto 2002; Bailin \& Steinmetz 2005; Allgood et al. 2006; Kuhlen et al. 2007; Faltenbacher et al. 2009; Schneider et al. 2012). While some early works found that the dark matter haloes are more spherical in the centre (e.g. Frenk et al. 1988), more recent studies agree that dark matter haloes become more spherical with increasing radius. There is, however, some disagreement on how the alignment of a halo changes with radius; i.e. $\theta_{\mathrm{H}}\left(R_{1}, R_{2}\right)$, see Figure 2 and Table 1.

For example, Schneider et al. (2012) investigated dark matter haloes using both the Millennium 1 (ME1, Springel et al. 2005) and Millennium 2 (ME2, Boylan-Kolchin et al. 2009) simulations. ME1 has a particle mass resolution of $8.6 \times 10^{8} h^{-1} \mathrm{M}_{\odot}$ and ME2 has a particle mass resolution a little over two orders of magnitude higher at $6.89 \times 10^{6} h^{-1} \mathrm{M}_{\odot}$. This study covered dark matter haloes over a range of masses from $10^{10}-2 \times 10^{14} h^{-1} \mathrm{M}_{\odot}$ and a wide range of halo radii $\left(0.1 R_{\text {vir }}-R_{\text {vir }}\right)$. They used the reduced inertia tensor with an elliptical weighting function to measure the shapes of the haloes at different radii. In addition to showing that dark matter haloes became more spherical with increasing radius, they also showed that the haloes became more spherical with decreasing halo mass at a fixed fraction of the virial radius. Figure 9 shows the internal alignments of individual dark matter haloes as a function of radius and halo mass at a redshift of $z=0.5$, which corresponds to an $R_{\mathrm{vir}}$ of $R_{131}$ (see Section 4.1.1). They found that the semimajor axes of the dark matter haloes had a mean misalignment angle of $\theta_{H}\left(R_{1}, R_{2}\right) \simeq 20^{\circ}$ between the inner and outer radii. There was a large scatter on the overall alignment distribution and while the distribution was skewed toward small misalignment angles, around $25 \%$ of the haloes had the semi-major axis of the outer halo perpendicular to the semi-major axis of the inner halo. More massive haloes tended to have less misalignment between the inner and outer radii. The authors noted that while the highest halo mass bin that could be investigated with both simulations had good agreement in halo alignments between the two simulations, this agreement decreased with decreasing halo mass bins. This was likely due to substructure contamination in ME1, since its lower resolution made it difficult to accurately remove all substructure particles, likely resulting in the shape measurements spuriously detecting more twisted inner and outer haloes.

Kuhlen et al. (2007) investigated a single very high particle mass resolution (particle mass $\simeq 20,000 h^{-1} \mathrm{M}_{\odot}$ ), isolated Milky-Way scale halo and also used the reduced inertia tensor with an elliptical weighting function to measure the shape of their halo, in addition to measuring the shape with the unweighted kinetic energy tensor (see Equation 35). Both measurements produced a prolate halo, however the velocity shape (from the kinetic energy tensor) was far more spherical than the mass distribution. Additionally, the halo became slightly more spherical in the outer radii in both cases. The semi-major axis of the halo was aligned to $1^{\circ}$ at all radii. However, as there was only one halo in this study, it is possible that this is a statistical fluctuation corresponding to one of the (not entirely rare) well-aligned haloes found in Schneider et al. (2012). Additionally, since the halo did not experience any major mergers from $z=1.7$, is was not subject to tidal interactions, making it much more likely to be well aligned.

It is still worth remembering that the mass resolution of Kuhlen et al. (2007) was significantly higher than in Schneider et al. (2012), so some of the twisting in the ME2 haloes may be a resolution effect. However, this is unlikely to account for all of the twisting measured and the general consensus in the field follows the results of Schneider et al. (2012), that dark matter haloes may experience misalignments between the inner and outer halo. This is further confirmed with the addition of baryons (see Section 5.1.1).

It is common to measure the alignment of the halo angular momentum with the halo axes, $\theta_{\lambda_{\mathrm{H}} \mathrm{H}}$. There is a strong consensus that the angular momentum of a halo is aligned parallel with the semi-minor axis and perpendicular to the semi-major axis of the halo mass distribution, as illustrated in Figure 10 (e.g. Barnes \& Efstathiou 1987; Warren et al. 1992; Bullock et al. 2001; Bailin \& Steinmetz 2005; Avila-Reese et al. 2005; Allgood et al. 2006; Shaw et al. 2006; Ragone-Figueroa \& Plionis 2007; Bett et al. 2007; Paz et al. 2008). Bailin \& Steinmetz (2005) found a median misalignment of $25^{\circ}$ between the semi-minor axis and the angular momentum, and a stronger alignment trend in the central $0.25 R_{\text {vir }}$ with the strength of the alignment increasing as a function of halo mass. There was also evidence 


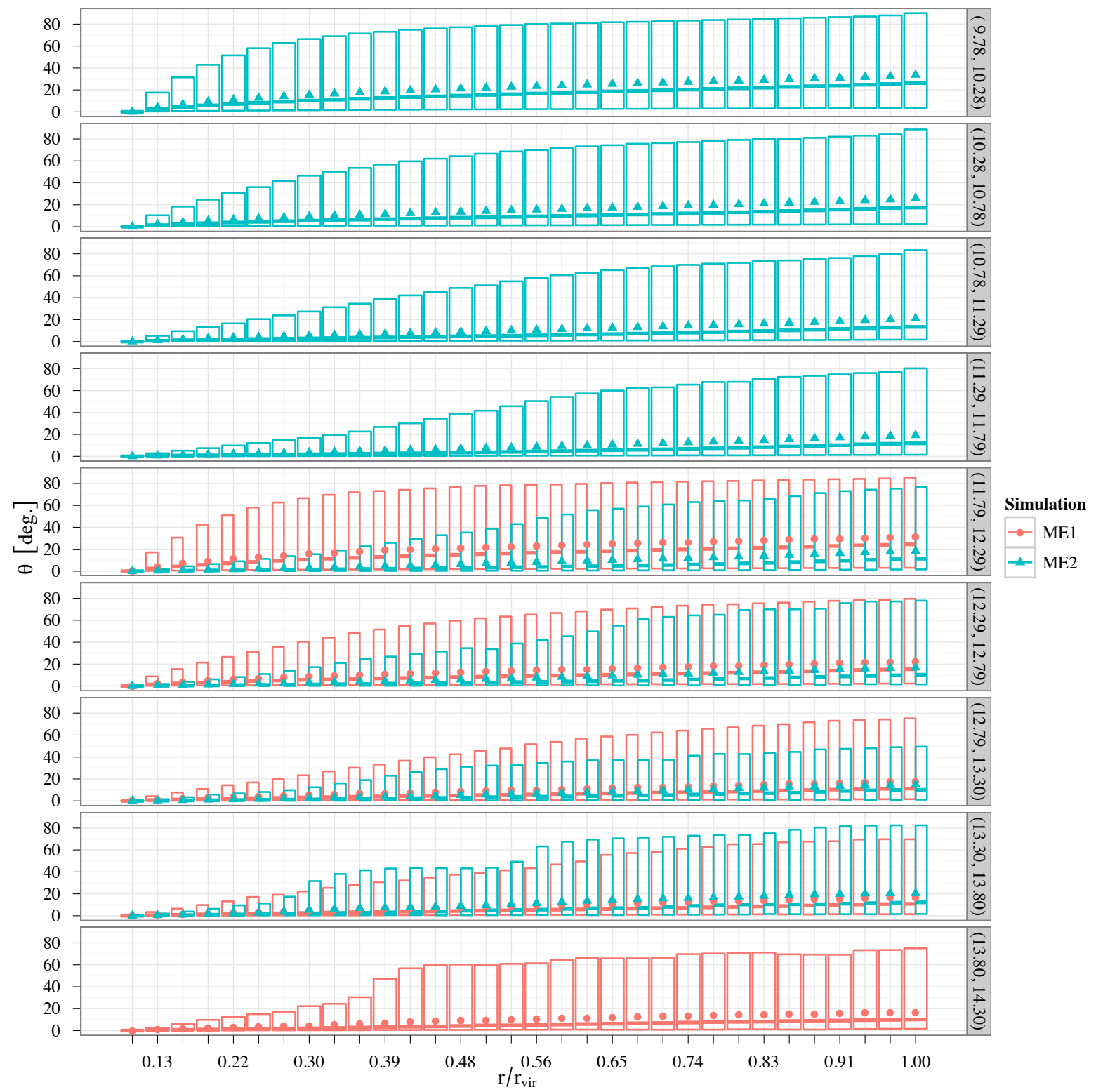

Fig. 9.-: Angle between the major axes at different radii within individual haloes, $\theta_{\mathrm{H}}\left(R_{1}, R_{2}\right)$ (see Figure 2 and Table 1), at $z=0.5$. The lower resolution simulation, Millennium 1, is shown in red, while the higher resolution simulation, Millennium 2, is shown in blue. Points indicate mean angles at a given radius, while boxes show the $25 \%-75 \%$ central quartile range with the median of the distribution denoted by a horizontal line within each box. Each panel shows a different dark matter halo mass range; this is shown in the grey box on the right with units of $\log _{10}\left(M_{200} / h^{-1} \mathrm{M}_{\odot}\right)$. The halo radius on the x-axis is normalized by $R_{\mathrm{vir}}$, which is $R_{131}$ at this redshift (see Section 4.1.1). (C) SISSA Medialab Srl. Reproduced by permission of IOP Publishing from Schneider et al. (2012). All rights reserved. 


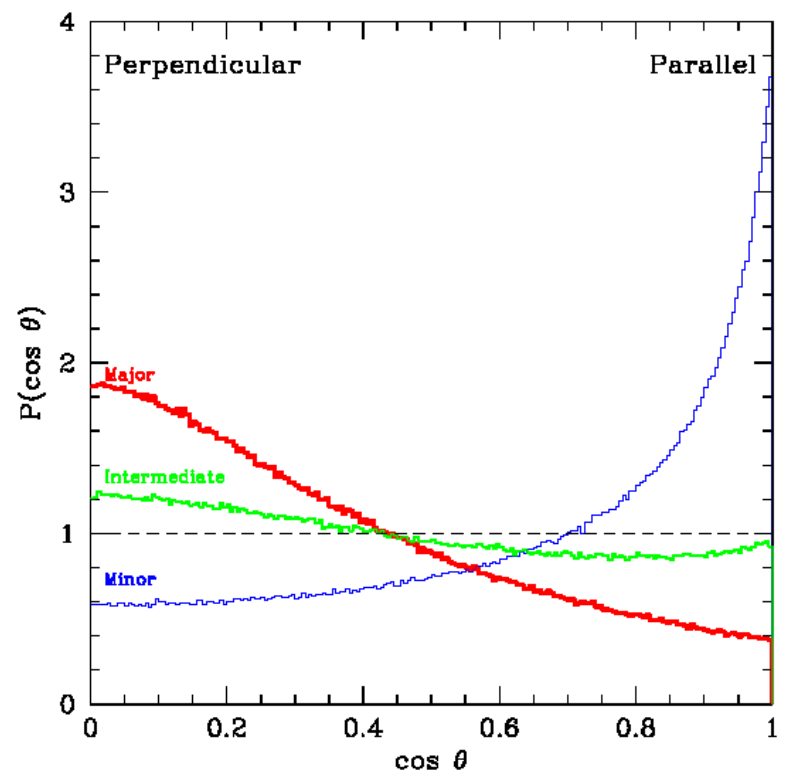

Fig. 10.-: Normalized histograms of the cosines of the angle between the angular momentum vector and the major (thick red line), intermediate (medium green line) and minor (thin blue line) axes of the dark matter haloes. A random distribution would be a flat line at $p(\cos \theta)=1$. Reproduced with permission from Bett et al. (2007).

to suggest that haloes containing a high level of substructure showed a stronger alignment between their angular momentum and semi-minor axes compared to haloes without substructure (Ragone-Figueroa \& Plionis 2007). Dark matter haloes can gain angular momentum through merger activity (e.g. Vitvitska et al. 2002; Maller et al. 2002), and haloes with more substructure have undergone more recent mergers. These mergers occurred preferentially in the plane defined by the halo semi-major/intermediate axes (see Section 4.2.5), adding to the angular momentum in the direction of the semi-minor axis.

Bett \& Frenk (2012) investigated significant, rapid changes to the direction of the angular momentum vector, which they termed 'spin flips'. The study focused on Milky Way sized dark matter haloes $\left(\sim 10^{12-12.5} h^{-1} \mathrm{M}_{\odot}\right.$ at $\left.z=0\right)$ that were relaxed. They found that these spin flips occurred in dark matter haloes regardless of whether they had experienced a major merger event in their lifetime. Instead, the spin flips were caused by strong tidal forces from events like minor mergers or even flybys of neighbouring haloes. They showed that $10.5 \%$ of their haloes had at least one spin flip of $>45^{\circ}$ within a timescale of $0.5 \mathrm{Gyrs}$ and that $10.1 \%$ of these occurred without a corresponding major merger event. They also investigated the spin flips in the inner $0.25 R_{\mathrm{vir}}$ and found that $58.5 \%$ of the haloes experienced a spin flip of $>45^{\circ}$ and that all but one of these occurred without a major merger event. This result is particularly interesting as spin flips of the inner dark matter halo may disrupt or morphologically transform galactic discs that form in this region (e.g. Okamoto et al. 2005; Romano-Díaz et al. 2009; Scannapieco et al. 2009).

Bullock et al. (2001) considered the alignment between the angular momentum vectors in the inner and outer half-haloes within the virial radius. Between $70 \%$ and $90 \%$ of the haloes were aligned to a greater degree than $\cos \left[\theta_{\lambda_{\mathrm{H}}}\left(R_{1}, R_{2}\right)\right]=0.7\left(\sim 45^{\circ}\right)$, with most showing significantly lower misalignments. Similar alignment results were seen by Bailin \& Steinmetz (2005), who considered the alignments at several reference radii. They found that the alignment became progressively worse as $R_{1}$ and $R_{2}$ were further separated, and that the median misalignment angle between the angular momentum vectors in the innermost and outermost regions was $\sim 50^{\circ}$.

Bailin \& Steinmetz (2005) also found that the internal alignment of angular momentum was worse for higher mass haloes. This is likely related to hierarchical structure formation; the most massive haloes formed most recently and 
are likely to have experienced a major merger more recently than smaller mass haloes, which perturbs the angular momentum of the halo.

\subsubsection{Halo-subhalo alignments}

In a Universe where structures form hierarchically (see e.g. Press \& Schechter 1974; Bond et al. 1991), it is expected that dark matter haloes will contain substructures or satellites. In the hierarchical formation scenario, at high redshifts low-mass objects begin to collapse. Through continuous accretion of dark matter over time, they increase their mass. They also undergo mergers with other haloes, and in this process the smaller halo will lose its loosely bound outer layers while the denser core survives as a subhalo within its new host halo. The alignment (shape, spin and angular momentum) and distribution of dark matter subhaloes within their host halo has been the subject of many $N$-body studies (e.g. Tormen 1997; Knebe et al. 2004; Kuhlen et al. 2007; Faltenbacher et al. 2008; Pereira et al. 2008; Knebe et al. 2008a,b; Angulo et al. 2009; Ocean Wang et al. 2014).

One of the alignment signals investigated is the orientation of the subhalo distribution within the shape of the host dark matter halo, $\theta_{\mathrm{BH}}$, see Figure 3a and Table 1 (e.g. Knebe et al. 2004; Faltenbacher et al. 2008; Kuhlen et al. 2007; Ocean Wang et al. 2014). Most studies used different versions of the inertia tensor (see Equation 33 and the surrounding text) to determine the axes of their host haloes. The exception was Ocean Wang et al. (2014) who determined the axes of the host haloes at given local mass overdensities following the method of Jing \& Suto (2002) (see Equation 34 and surrounding text). Most studies determined the shape of the subhalo distribution through the lines connecting the centre of the host halo with each subhalo and calculated the alignment as a shape-position alignment, $\langle|\boldsymbol{l} \cdot \hat{\boldsymbol{r}}|\rangle$ (Knebe et al. 2004; Faltenbacher et al. 2008; Ocean Wang et al. 2014). While Kuhlen et al. (2007) determined the axes of the subhalo distribution ellipsoid through diagonalising the weighted moment of inertia tensor constructed from the positions of each subhalo, finally treating the alignment as a shape-shape alignment. The resulting alignments determined whether the subhalo distribution ellipsoid (or ellipse if projected on to a 2D observer plane; e.g. Ocean Wang et al. 2014) was random within the host or if it exhibited some anisotropic signal.

Instead of calculating the alignment with the orientation angle of the host halo, Ocean Wang et al. (2014) assumed an orientation for an imagined central galaxy, $\theta_{\mathrm{BC}}$ (see Figure $3 \mathrm{a}$ and Table 1). This orientation either followed the inner, intermediate or outer host halo orientations. Their results showed that while the satellite distribution aligned with the "galaxy" semi-major axis at all orientations, the alignments increased in strength for orientations with the inner, intermediate and outer haloes respectively (implying an alignment, $\theta_{\mathrm{BH}}$, with the outer host halo semi-major axis). When comparing with observations, the alignment signal was too strong when the galaxy followed the outer halo orientation, and much closer to the observations when the galaxy followed the inner halo orientation or had a misalignment drawn from a Gaussian distribution with a mean of $0^{\circ}$ and standard deviation of $25^{\circ}$.

There are differences within all of these studies on how the subhalo population was selected. Additionally, a number of different halo finding algorithms were employed and each had different particle or mass cutoffs. Regardless of these differences, the consensus is that the orbits of subhaloes within a host halo are strongly anisotropic (not random) and the semi-major axes of the subhalo distribution ellipsoids preferentially align with the semi-major axis of the outer host halo. The question now is why subhaloes exhibit this alignment and whether it is the result of anisotropic infall or if the subhaloes have an isotropic infall and then experience dynamic effects that lock in the alignment. Given that the outer host halo may be significantly misaligned with the inner host halo (see Section 4.2.2), which would have an impact on the alignment of the subhalo population, this might suggest that dynamical effects are the cause of subhalo distribution alignments. However, haloes merge preferentially along the direction of their host filament (see Section 4.2.5), which would add an element of anisotropic infall.

The alignment of the subhalo distribution with the host halo spin axis, $\theta_{\lambda_{\mathrm{H}} \mathrm{B}}$, was investigated by Angulo et al. (2009). They found that the subhalo distribution was aligned perpendicular to the spin axis. As mentioned in Section 4.2.2, the angular momentum vector is preferentially perpendicular to the major axis of the host halo, indicating that this work is consistent with the results above.

Another quantity that is studied is the alignment of a subhalo with the centre of mass of the host halo, $\theta_{\mathrm{Sh}}$, see Figure 3a and Table 1 (e.g. Kuhlen et al. 2007; Faltenbacher et al. 2008; Knebe et al. 2008a,b; Pereira et al. 2008). The majority looked at the 3D shapes of the subhaloes but Knebe et al. (2008b) investigated the 2D projection on to 

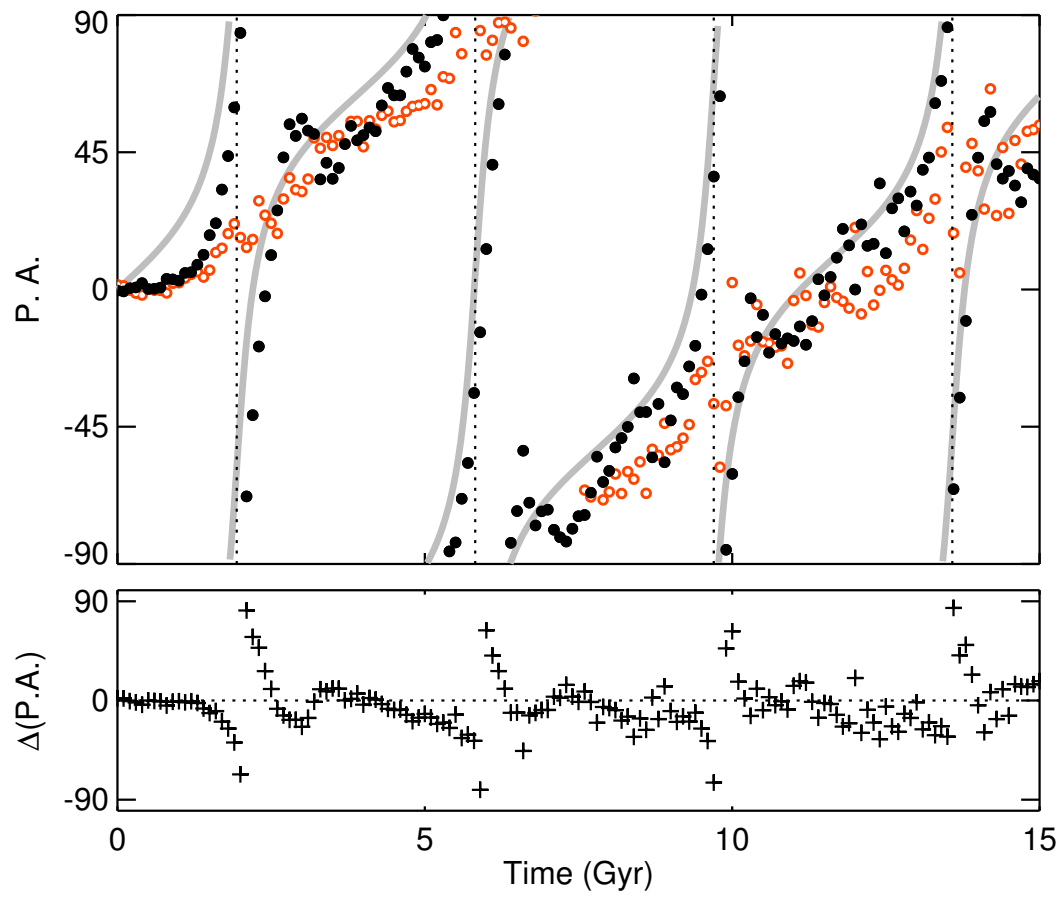

Fig. 11.-: Top: Stellar and dark matter position angles (P.A.) vs. time for an eccentric orbit. Grey lines indicate the direction to the cluster centre and dotted vertical lines represent pericentre passages. While the dark matter particles (black dots) appear to follow the orbital motion closely, varying their rotation speed to match the orbital velocity changes, the stellar particles (red circles) appear to be locked in uniform figure rotation. Bottom: Misalignment between the orientation of the dark matter and stars $\mid \Delta$ P.A.| as a function of orbital time. The misalignment peaks immediately after each pericentre passage, and remains small for the rest of the orbit. (C) AAS. Reproduced with permission from Pereira \& Bryan (2010).

the observers plane. The studies all agreed that there is a strong preferential radial alignment of subhaloes with the centre of mass of the host. Faltenbacher et al. (2008) investigated this trend over a wide range of scales and found, unsurprisingly, that this radial alignment was strongest on smaller scales and dropped off rapidly with increasing distance from the centre of the host halo. The evolution of this alignment showed that while there is a strong preference for a nearly radial alignment, there are some deviations throughout the orbit of the subhalo around its host.

Pereira \& Bryan (2010) took a different approach to study the alignments of satellite galaxies within dark matter haloes. They simulated a multi-component, $N$-body (stars+dark matter) satellite, orbiting an external, analytical potential (which simulated the host cluster sized halo), and showed that the stellar component of the satellite reacted significantly slower to the tidal torque from the host halo than the dark matter, producing a radial twisting of the satellite that depended on the eccentricity of the orbit. If the satellite was in a circular orbit, both dark matter and stars were tidally locked (semi-major axis pointing toward the centre of mass) but the stars took approximately twice as long as the dark matter to lock to the external potential. In such orbit, the stars and dark matter were always aligned with each other when measured at the same radius. By contrast, in very eccentric orbits the change in torque was too rapid to tidally lock the stars, which figure-rotated around the potential, while the dark matter maintained a radial alignment except at pericentre where a sudden misalignment occurred (Figure 11, top panel). Additionally, after a brief period of influence from the host halo, the stars and dark matter aligned with each other, except for short periods surrounding the pericentre passage (Figure 11, bottom panel). Overall, Pereira \& Bryan (2010) also found that there is a strong preference for nearly radial alignments but they also showed that the magnitude of these deviations depends on the 


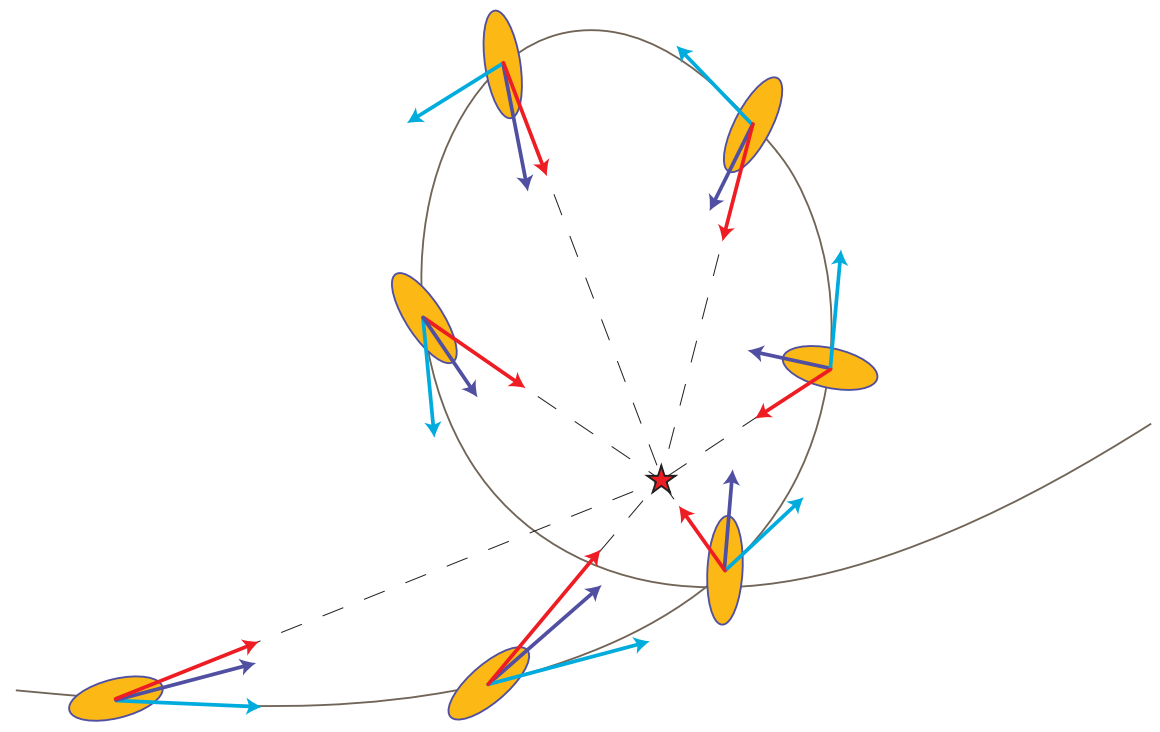

Fig. 12.-: Sketch of a subhalo in orbit around its host. The centre of mass of the host is represented by a star. The radial direction is drawn as a red vector, the light blue vector indicates the orbital direction, and the dark blue vector is the direction of the major axis of the subhalo. The red and dark blue vectors are generally very close, apart from a short mismatch at pericentre caused by the high orbital velocities. The dark blue and light blue vectors are close before pericentre, but almost orthogonal to each other in the second part of the orbit. (c) AAS. Reproduced with permission from Pereira et al. (2008).

eccentricity of the orbit.

Kuhlen et al. (2007); Pereira et al. (2008) and Pereira \& Bryan (2010) used tidal torque theory to explain the motion of a subhalo around the host; this is illustrated in Figure 12. A subhalo is typically in an eccentric orbit. As it approaches pericentre, the subhalo generally points toward the centre of the host halo mass because it is being tidally torqued in the direction of the potential gradient, which is also close to the direction of motion. As the subhalo reaches pericentre, the torquing is less effective due to its high velocity at this point, resulting in a lower radial alignment signal. The torquing continues throughout the orbit, which keeps the subhalo semi-major axis largely aligned with the centre of mass of the host. The misalignment of the subhalo semi-major axis with the orbital direction initially increases before coming back into alignment after passing the apocentre. As the subhalo approaches pericentre again, a new cycle begins.

The signals found in the simulations were typically stronger than those in observations (that measure the alignments of the luminous satellites) and Knebe et al. (2008b) also attempted to explain this through tidal torque theory. Since the subhaloes are subject to strong tidal torquing as they pass through apocentre, the loosely bound outer particles will be more highly distorted than the inner particles. Consequently, the shape of the outer subhalo could be more easily pulled into alignment with the center of mass of the host than the inner region at the centre of the subhalo (where the luminous satellite would reside). This would result in the luminous satellite and the inner region of the dark matter halo having a weaker alignment with the centre of mass of the host than the outer subhaloes.

\subsubsection{Halo-halo alignments}

Determining whether dark matter haloes have a tendency to align with nearby haloes has been the focus of a number of studies (e.g. Barnes \& Efstathiou 1987; Croft \& Metzler 2000; Heavens et al. 2000; Onuora \& Thomas 2000; Jing 2002; Bailin \& Steinmetz 2005; Hopkins et al. 2005; Altay et al. 2006; Schneider et al. 2012). There are two different kinds of alignments measured - the first is a direct alignment, $\Theta_{\mathrm{HH}}$, that measures whether neighbouring halo axes tend 
to point in the same direction; see Equation 46 for 3D alignments and Equation 50 for 2D alignments. The second alignment, $\Theta_{\mathrm{Hh}}$, determines whether haloes tend to point in the direction of their neighbours; see Equation 47). Both alignments are shown in Figure $3 \mathrm{~b}$ and Table 1 and are discussed below.

Many studies showed that the semi-major axes of group and cluster sized haloes $\left(M_{\mathrm{h}} \gtrsim 10^{13} h^{-1} \mathrm{M}_{\odot}\right)$ preferentially point in the same direction in a 3D analysis (e.g. Barnes \& Efstathiou 1987; Faltenbacher et al. 2002; Kasun \& Evrard 2005; Hopkins et al. 2005). There was evidence showing this signal to be significant out to $30 h^{-1} \mathrm{Mpc}$ in the simulations (e.g. Faltenbacher et al. 2002; Hopkins et al. 2005; Kasun \& Evrard 2005). Bailin \& Steinmetz (2005) investigated the alignments, $\Theta_{\mathrm{HH}}$, using predominantly galaxy mass haloes and measured an alignment signal that was lower in amplitude than the works above, which they attributed to the lower mass range of their haloes. This assumption was confirmed in a later investigation by Schneider et al. (2012) that measured the alignment over a wide range of halo masses from subhaloes to clusters $\left(10^{10}<M_{\mathrm{h}}<2 \times 10^{14} h^{-1} \mathrm{M}_{\odot}\right)$. This study showed that there was a tendency for neighbouring halo semi-major axes to point in the same direction in all mass ranges, but the strength of the alignment was a strongly increasing function of halo mass.

In similar studies, Croft \& Metzler (2000); Heavens et al. (2000) and Jing (2002) investigated projected 2D axis alignments of cluster sized dark matter haloes. Projections provide a lower limit on the alignment signal (since projections necessarily lose information about the system). However, Jing (2002) showed that the results in Croft \& Metzler (2000) and Heavens et al. (2000) further underestimated the correlations as their shape measurements included haloes with as few as 20 particles, which could lead to a factor of two underestimate of ellipticity correlation. Using a minimum of 160 particles for their final shape measurements, Jing (2002) showed that the ellipticity correlations had a high enough amplitude to contaminate both deep and wide weak lensing surveys.

A number of works considered the alignment, $\Theta_{\mathrm{Hh}}$, of the orientation of a halo with the direction to the centre of mass of a neighbouring halo, (e.g. Onuora \& Thomas 2000; Faltenbacher et al. 2002; Hopkins et al. 2005; Kasun \& Evrard 2005; Altay et al. 2006; Schneider et al. 2012). This alignment signal tended to be stronger than the halo axis alignments (e.g. Faltenbacher et al. 2002; Hopkins et al. 2005; Kasun \& Evrard 2005; Schneider et al. 2012), and was also a strongly increasing function of halo mass (e.g. Bailin \& Steinmetz 2005; Schneider et al. 2012). The signal was detected out to $\geq 100 h^{-1} \mathrm{Mpc}$ for group and cluster mass haloes $\left(M_{\mathrm{h}} \gtrsim 2 \times 10^{13} h^{-1} \mathrm{M}_{\odot}\right)$ (e.g. Faltenbacher et al. 2002; Hopkins et al. 2005; Kasun \& Evrard 2005). Hopkins et al. (2005) also studied cluster halo alignments as a function of redshift and showed that the alignments were greater at early times and that aligned halo pairs were more likely to be connected by a filament than unaligned halo pairs. They found this result was consistent with the anisotropic merging and infall scenario, where haloes form through mergers and accretion of dark matter traveling coherently along the direction of the large-scale filaments, such that the alignments should be present from the time of halo formation.

Lee et al. (2005) attempted to provide an alternative explanation to the anisotropic infall model, arguing that the model is merely qualitative and that primordial alignments should be damped over time by non-linear processes. Anisotropic infall has been used to explain the observational result that brightest cluster galaxies and dark matter haloes have their semi-major axes preferentially aligned. To mimic this scenario in the absence of baryons, they identified the largest central subhalo within the cluster-sized dark matter haloes to represent the central galaxy and investigated the alignment, $\theta_{\mathrm{SH}}$. In their alternative model, interaction between the host halo tidal field and the subhalo should account for the observed alignments, assuming that the subhalo angular momentum is aligned parallel to the subhalo semi-minor axis. Their simulations showed that the semi-minor axes of the subhaloes were preferentially perpendicular to the semi-major axis of the host haloes, in agreement with observations and the predictions of their alternative model. However, in order to rule out the anisotropic infall model, they conceded that they needed to measure the angular momentum alignments directly but their simulations lacked the resolution required to do this.

When investigating spin-spin alignments, $\Theta_{\lambda_{\mathrm{H}} \lambda_{\mathrm{H}}}$, tidal torque theory suggests that neighbouring haloes should experience some alignment of their spin vectors, . Trowland et al. (2013) showed that neighbouring halo spins had a weak parallel alignment to each other, but only for halo separations under $0.3 h^{-1} \mathrm{Mpc}$. On these scales, only subhaloes in massive clusters would be able to exhibit alignments. The scale of this halo separation is so small that this is likely why other lower resolution studies did not detect any clear alignment signal (Barnes \& Efstathiou 1987; Porciani et al. 2002; Faltenbacher et al. 2002; Bailin \& Steinmetz 2005). By contrast, Hatton \& Ninin (2001) found significant but weak alignments on scales from $1-30 h^{-1} \mathrm{Mpc}$ and Hahn et al. (2007a) also found a correlation in alignments of spins 
for massive haloes in clusters. However, in an extension of this work using a different selection criteria for their haloes, Hahn et al. (2007b) found no halo-halo spin correlation. They used a more stringent selection criteria to remove haloes that had not yet relaxed. These systems were mostly two close neighbours that were either in the process of merging or had been spuriously linked into a single halo. They also removed the haloes where the distance of the most bound particle from the centre of mass exceeded a quarter of the largest distance between the outermost particle in the halo and the centre of mass (since the most bound particle in a relaxed halo would normally reside close to the centre of mass). This cleaned catalogue modified the spin parameter distribution, and hence reversed their earlier detection of spin alignments. Since halo selection criteria can have a significant effect on results, this, and the fact that they measured the spins of haloes with as few as 20 particles, may offer reasonable explanations for why Hatton \& Ninin (2001) found alignments in their simulations.

\subsubsection{Halo-LSS alignments}

$\mathrm{N}$-body simulations on a cosmological scale have shown that structure formation (i.e. gravitational collapse) occurs first in sheets. Filaments then form and matter in these filaments collapses into dark matter haloes. These dark matter haloes move along the filaments toward intersections between multiple filaments that are knots of potential minima where cluster haloes are formed. See Section 4.1.4 for more information and details on some cosmic web classification schemes.

There are a number of studies that measure the shape alignment of a halo with the surrounding large-scale structure; $\theta_{H W}, \theta_{H F}, \theta_{S H}{ }^{12}, \theta_{\mathrm{Hv}}$ (e.g. Patiri et al. 2006; Hahn et al. 2007b; Brunino et al. 2007; Cuesta et al. 2008; Zhang et al. 2009; Paz et al. 2011; Libeskind et al. 2013; Forero-Romero et al. 2014). The consensus is that shape alignments with the LSS are stronger and more robust to measure than angular momentum/spin correlations. There is a consistently strong parallel alignment of the semi-major axis of a halo with its host sheet plane, the semi-major axis of its host filament or cluster or perpendicular to the radial direction of the centre of voids (the semi-minor axis of the haloes tends to be parallel to the radial direction of voids) in all but the lowest mass haloes. This alignment increases in strength with increasing halo mass. Forero-Romero et al. (2014) traced the shape of the structure using both the tidal shear field (which they term the T-web; Equation 43) and velocity field (V-web; Equation 44). However, they did not divide their simulation into distinct web elements using a threshold $\lambda_{t h}$, instead choosing to classify the alignments directly with respect to the eigenvectors $\hat{e}_{1}$ and $\hat{e}_{3}$. For simplicity of reporting results, they defined a strong alignment with $\hat{e}_{3}$ to be a strong alignment, $\theta_{\mathrm{HF}}$ (see Figure 4b), with a filament, while a strong alignment with $\hat{e}_{1}$ is an antialignment, $\theta_{\mathrm{HW}}$ (see Figure 4a and Table 1), with a sheet. This choice has the benefit of being independent of $\lambda_{t h}$ for each classification scheme, however the signal may become diluted when mixing of the signal occurs between environments. Despite this unusual classification scheme, their results for the tidal shear field matched previous works. However in the momentum-based velocity field measurement, they found an anti-alignment of haloes with sheets for haloes $M_{\mathrm{h}}>10^{12} h^{-1} \mathrm{M}_{\odot}$.

Many works investigated the spins of a haloes as a function of environment (e.g. Aragón-Calvo et al. 2007; Hahn et al. 2007a,b; Brunino et al. 2007; Sousbie et al. 2008; Zhang et al. 2009; Codis et al. 2012; Trowland et al. 2013; Libeskind et al. 2013; Aragon-Calvo \& Yang 2014; Forero-Romero et al. 2014). There is consensus that this measurement is far less robust than the alignments with halo shape and results are more dependent on measurement algorithm, simulation and environment definition. There is little $N$-body simulation literature for angular momentum alignments around voids, $\theta_{\lambda_{\mathrm{H}}}$, directly, although comparisons may be made with alignments of sheets since these reside at the boundaries of the void surfaces and in the case where the void is modelled as spherical, the alignment would simply be perpendicular to the sheet alignment. Heymans et al. (2006); Patiri et al. (2006) and Brunino et al. (2007) investigated the void alignments directly and concluded that there were no angular momentum alignments around voids and the orientation of the angular momentum of the haloes was random. By contrast Cuesta et al. (2008) ${ }^{13}$ did find alignments, where the angular momentum vector of haloes located in a shell at the void surface was aligned preferentially perpen-

\footnotetext{
${ }^{12}$ Note that the subscript ' $\mathrm{H}$ ' in this alignment is the cluster cosmic web element and the ' $\mathrm{S}$ ' represents the galaxy-sized and smaller halo substructure within the cluster.

${ }^{13}$ While this simulation includes hydrodynamics, the results are focused on dark matter haloes and appear to be independent of the baryons, hence its inclusion in this section.
} 


\begin{tabular}{|c|c|c|c|c|c|}
\hline Author & Web Method & $\begin{array}{c}\text { Spatial Scale } \\
h^{-1} \mathrm{Mpc}\end{array}$ & Along & Alignment & Mass dependence \\
\hline \multirow[t]{4}{*}{ Forero-Romero et al. (2014) } & T-Web & $0.5-1$ & $\hat{e}_{3}$ (filament) & - & $>10^{12} h^{-1} \mathbf{M}_{\odot}$ \\
\hline & & & $\hat{e}_{3}$ (filament) & none & $<10^{12} h^{-1} \mathrm{M}_{\odot}$ \\
\hline & & & $\hat{e}_{1}($ wall $)$ & none & $>10^{12} h^{-1} \mathbf{M}_{\odot}$ \\
\hline & & & $\hat{e}_{1}($ wall $)$ & none & $<10^{12} h^{-1} \mathrm{M}_{\odot}$ \\
\hline \multirow[t]{4}{*}{ Forero-Romero et al. (2014) } & V-Web & $0.5-1$ & $\hat{e}_{3}$ (filament) & none & $>10^{12} h^{-1} \mathrm{M}_{\odot}$ \\
\hline & & & $\hat{e}_{3}$ (filament) & none & $<10^{12} h^{-1} \mathrm{M}_{\odot}$ \\
\hline & & & $\hat{e}_{1}($ wall $)$ & + & $>10^{12} h^{-1} \mathrm{M}_{\odot}$ \\
\hline & & & $\hat{e}_{1}($ wall $)$ & none & $<10^{12} h^{-1} \mathrm{M}_{\odot}$ \\
\hline \multirow[t]{3}{*}{ Libeskind et al. (2013) } & V-Web & 1 & filament & - & $>10^{12} h^{-1} \mathbf{M}_{\odot}$ \\
\hline & & & filament & + & $<10^{12} h^{-1} \mathbf{M}_{\odot}$ \\
\hline & & & wall & ++ & all masses \\
\hline \multirow[t]{2}{*}{ Trowland et al. (2013) } & Hessian density & $2-5$ & filament & - & $>5 \times 10^{12} h^{-1} \mathrm{M}_{\odot}$ \\
\hline & & & filament & + & $<5 \times 10^{12} h^{-1} \mathrm{M}_{\odot}$ \\
\hline \multirow[t]{3}{*}{ Codis et al. (2012) } & Morse Theory \& T-Web & $1-5$ & filament & -- & $>10^{12.5} h^{-1} \mathrm{M}_{\odot}$ \\
\hline & & & filament & ++ & $<10^{12.5} h^{-1} \mathrm{M}_{\odot}$ \\
\hline & & & wall & ++ & all masses \\
\hline \multirow[t]{2}{*}{ Zhang et al. (2009) } & Hessian density & 2.1 & filament & ++ & if anticorrelated with shape \\
\hline & & & filament & -- & if correlated with shape \\
\hline \multirow[t]{4}{*}{ Aragón-Calvo et al. (2007) } & Hessian density & - & wall & ++ & $>10^{12} h^{-1} \mathbf{M}_{\odot}$ \\
\hline & & - & wall & + & $<10^{12} h^{-1} \mathbf{M}_{\odot}$ \\
\hline & & - & filament & - & $>10^{12} h^{-1} \mathrm{M}_{\odot}$ \\
\hline & & - & filament & + & $<10^{12} h^{-1} \mathrm{M}_{\odot}$ \\
\hline \multirow[t]{3}{*}{ Hahn et al. (2007b) } & Tidal Web & 2.1 & filament & - & none \\
\hline & & & wall & ++ & $>10^{12} h^{-1} \mathrm{M}_{\odot}$ \\
\hline & & & wall & + & $<10^{12} h^{-1} \mathrm{M}_{\odot}$ \\
\hline
\end{tabular}

Table 2:: Angular momentum alignment with the cosmic web, where $\hat{e}_{1}$ is the major and $\hat{e}_{3}$ is the minor eigenvector of the corresponding tensor (velocity or shear). Summary of theoretical results provided by similar analysis methods. $(--)++$ indicates a strong (anti-)alignment and (-)+ indicates a weak (anti-)alignment. Reproduced with permission from Forero-Romero et al. (2014).

dicular to the direction of the centre of the void. These alignments decreased rapidly with void radius, so taking a wide shell would mask the signal (leaving them actually in agreement with Heymans et al. 2006 who only used wide shells). They also showed a slight trend of increasing alignments with increasing halo mass. It is worth noting that this result is in contrast with the findings presented in Section 4.2.2, that the angular momentum of a halo is preferentially parallel to the semi-minor axis of the halo; Cuesta et al. (2008) found the semi-minor axis of their haloes was preferentially parallel to the radial direction to the centre of the voids so the angular momentum of their haloes is preferentially perpendicular to the semi-minor axis in this work. Given these discrepancies, further investigations would be required to clarify the alignments of the halo angular momentum vector around voids.

When considering the alignment, $\theta_{\lambda_{\mathrm{H}} \mathrm{W}}$, of the angular momentum of haloes with sheets, there is a trend for the angular momentum to be aligned parallel to the sheet (e.g. Aragón-Calvo et al. 2007; Hahn et al. $2007 \mathrm{~b}$; Zhang et al. 2009; Codis et al. 2012; Trowland et al. 2013; Libeskind et al. 2013). There is also a trend for the alignment, $\theta_{\lambda_{\mathrm{H}} \mathrm{F}}$, of the angular momentum of high mass haloes to be perpendicular to a filament, while lower mass haloes align parallel to the filament (e.g. Aragón-Calvo et al. 2007; Hahn et al. 2007a; Sousbie et al. 2008; Codis et al. 2012; Trowland et al. 2013; Aragon-Calvo \& Yang 2014) and the mass where this transition occurs is around $M_{0}^{S} \simeq 5 \times 10^{12} h^{-1} \mathrm{M}_{\odot}(\mathrm{e} . \mathrm{g}$. Hahn et al. 2007a; Aragón-Calvo et al. 2007; Codis et al. 2012; Trowland et al. 2013). Codis et al. (2012) investigated the redshift dependence of the transition mass and found that the halo transition mass $M_{\text {crit }}^{S}$ decreased with increasing redshift such that

$$
M_{\mathrm{crit}}^{S} \approx M_{0}^{S}(1+z)^{-\gamma_{s}}, \quad \gamma_{s}=2.5 \pm 0.2
$$

Looking at the alignments in terms of the halo model, Paz et al. (2008) found similar results. They calculated 
the dark matter correlation function parallel and perpendicular to the angular momentum vector of the haloes. They found that the halo angular momentum vectors aligned preferentially perpendicular to the mass distribution of the large-scale structure (the two-halo term) at high masses while low mass halo angular momenta may preferentially point parallel to the mass distribution on large scales. This inversion occurred around masses of $5.5 \times 10^{12.5} h^{-1} \mathrm{M}_{\odot}$, which is qualitatively consistent with the findings above.

In contrast to these findings Libeskind et al. (2013) disputed that the transition of the halo angular momentum vector alignment from perpendicular to the filament in high mass haloes to parallel in low mass haloes occurs only in filaments. They showed evidence for this transition occurring in all environments, with the mass at which the transition occurs decreasing with environment type from clusters down to voids (e.g. in voids, haloes of intermediate mass transition the direction of their angular momentum from perpendicular to the void centre to parallel to the void centre with decreasing mass). In further contrast, Forero-Romero et al. (2014) found results that only broadly matched many of these earlier works. For web elements defined in the V-web, they found that the angular momentum of haloes with masses above $10^{12} h^{-1} \mathrm{M}_{\odot}$ tended to align along walls, without any clear trend with respect to filaments, while the alignment signal disappeared for halo masses below $10^{11} h^{-1} \mathrm{M}_{\odot}$. In the T-web, they similarly found no evidence for any alignment of haloes with masses below $10^{12} h^{-1} \mathrm{M}_{\odot}$, and only a weak signal for alignment at higher mass, with a trend for the angular momentum to lie perpendicular to filaments. The discrepancies in this work may be the result of the chosen web classification scheme diluting an already weak signal even further by mixing environments, or the alignments could have a high sensitivity on small scales to the method used to construct the cosmic web (including numerical choices for interpolating the relevant fields). Forero-Romero et al. (2014) provided a helpful comparison of angular momentum alignments found in these similar works, which is reproduced in Table 2.

\section{3. $\quad N$-body simulations Roundup}

There is a wealth of literature that investigates the alignments of both the shapes and spins of dark matter haloes in $\mathrm{N}$-body simulations over a large range of scales. This section introduced the common techniques used to measure alignments in simulations and reviewed the existing literature on the alignments of dark matter haloes in $N$-body simulations.

Overall, the shape alignments were stronger and more robust than the spin alignments. It was established that the dark matter haloes have a strong tendency to be prolate and that the shape of the halo can change with radius. The subhalo distribution semi-major axes tend to align with the semi-major axis of the host dark matter halo and individual subhalo semi-major axes tend to point toward the centre of mass of the host dark matter halo throughout their orbit. Neighbouring dark matter haloes tend to have semi-major axes that are aligned parallel and a stronger tendency to point in the direction of neighbouring haloes (in the direction of the large-scale structure). This is consistent with the findings that the semi-major axes of dark matter haloes tend to align parallel with their host sheet plane, the semimajor axis of their host filament or cluster or perpendicular to the direction to the centre of the void. In general, shape alignments on all scales were stronger with increasing halo mass.

The literature on angular momentum and spin alignments had less consensus. A number of works found the distribution of halo spin parameters to be well fit by a lognormal distribution, while some works found that the lognormal was a poor fit. Further investigation with $N$-body simulations containing a large statistical sample (many thousands) of well resolved haloes would be required to resolve this issue. There was strong consensus that the angular momentum vector was aligned parallel with the semi-minor axis and perpendicular to the semi-major axis of the dark matter halo. Yet, there was also clear evidence that the haloes experience significant, rapid changes in the alignment of the angular momentum vector over time (spin flips) as a result of major and minor mergers and even flybys from other dark matter haloes. In addition, the direction of the angular momentum vector can change as a function of halo radius and these spin flips can occur in the inner radii as well as the outer radii. As events like mergers and flybys cause the angular momentum vector to flip, the selection criteria for the dark matter haloes included the studies has a strong influence on the findings. The frequent halo spin-flips are likely responsible for the less robust spin alignment results.

Nearby neighbouring haloes (with small separations) may show a weak alignment of their spin vectors in the same direction, but no alignments at larger separations. In the large-scale structure, the spin vectors of the dark matter haloes tend to be parallel with the plane of their host sheet, while there is no clear consensus on the orientation with voids. 
Although if sheets are considered the boundaries of (spherical) voids then it could be argued that the dark matter halo spin vectors are preferentially perpendicular to the direction to the centre of a void. A number of works found that dark matter halo spin vectors tended to be parallel with their host filament at low masses and transitioned to being perpendicular at masses above around $5 \times 10^{12} h^{-1} \mathrm{M}_{\odot}$, with this mass decreasing with increasing redshift. It is also possible that this transition occurs in all cosmic web elements with the mass where the transition occurs decreasing with environment type from clusters to filaments, sheets, and voids.

It is difficult to relate these studies to the real world because observations deal with luminous galaxies and we cannot currently measure the alignments of the dark matter haloes directly. In some studies, it was assumed that the orientation of the central region of the dark matter halo could be used as a proxy for the central galaxy. However, Section 5 shows that the introduction of baryons significantly changes the relative shapes and alignments of the dark matter haloes, particularly in the inner regions of the halo. Consequently, caution should be exercised when exclusively using $N$ body simulations to gain insight into galaxy alignments. Despite these limitations, $N$-body simulations continue to play an important role in studies of galaxy alignments. This is discussed further in Sections 6 and 7.

\section{Hydrodynamic simulations}

A significant shortcoming of $N$-body simulations in the study of galaxy dynamics or intrinsic alignments is that these simulations are concerned only with the evolution of structure under gravity. The particles used in the simulations interact only gravitationally, and any effects relating to baryon or gas physics are completely ignored. Detailed theoretical understanding of the (expected) shape and angular momentum/spin alignments between galaxies inside haloes and their host haloes (and combinations thereof) is fundamentally limited due to the not-well-understood role that gravitational collapse of the halo and baryonic physics has on the shapes of galaxies. In particular the feedback processes between galaxy and cluster-scale physics and the surrounding dark matter haloes is not well understood. Hydrodynamic ${ }^{14}$ simulations address this issue by treating the evolution of the gaseous component of the Universe using the methods of computational fluid dynamics. This approach enables the complex interactions of the different baryonic components (gas, stars, etc.) to be treated self-consistently with the dark matter, and on a much smaller scale. This enables simulations of the formation of galaxies within dark matter halos, and the ability to probe the complex physical processes that give rise to the visual properties of galaxies that we observe today.

Hydrodynamic simulations are a relatively new way to explore the intrinsic alignments of galaxy shapes and spins, in part because they are quite computationally intensive. The obvious advantage of using hydrodynamic simulations is that $N$-body simulations require some sort of semi-analytic model to identify the positions and orientations of galaxies (see Section 6), whereas in hydrodynamic simulations, the process of galaxy formation is naturally included. The disadvantage of using hydrodynamic simulations is that the relevant sub-grid physics that is needed to form realistic galaxies is not yet known. The term "sub-grid physics" is meant to include all physical processes that take place on a smaller scale than the resolution of the simulation, and therefore must be included with some model. This includes some important aspects of star formation, accretion onto supermassive black holes, radiative heating/cooling, and feedback from supernovae (important at low masses) and some other mechanisms at high masses (e.g., from AGN). While there are claims in the literature of increasingly realistic galaxy populations using hydrodynamic simulations (e.g. Khandai et al. 2015; Vogelsberger et al. 2014), the results still show some discrepancies with reality that require caution with their usage. For example, the results of the sub-grid physics are often tuned to match particular observables (e.g. the luminosity function) at a particular redshift (typically redshift zero), so extrapolation to different redshifts should therefore be treated with caution, as observations at high redshift are limited and matching these results is not guaranteed. Ideally, a full exploration of intrinsic alignments in hydrodynamic simulations would involve a comparison between several independent simulations with different implementations of sub-grid physics, to check how the results for galaxy intrinsic alignments depend on the details of galaxy formation and feedback. Unfortunately, such a comparison is not yet possible due to the tremendous expense of large-volume and high-resolution hydrodynamic simulations.

${ }^{14}$ Note that the terms "hydrodynamic" and "gasdynamic" are used interchangeably in the literature, although hydrodynamic is adopted in this review. 


\subsection{Small-volume hydrodynamic simulations}

In this section, small-volume and zoom hydrodynamic simulations are discussed.

\subsubsection{Internal alignments}

One very interesting study made possible with hydrodynamic simulations is how the baryonic galaxy aligns with its host dark matter halo, $\theta_{C H}$, and whether baryons have an effect on halo shape and alignment.

Broadly, studies agree that the addition of baryons significantly affects the shape of the host dark matter halo. Most agree that a galaxy forming in the centre of the halo will cause the halo to be more spherical overall and there is a consensus that haloes with galaxies tend toward oblateness, rather than the prolateness seen in $\mathrm{N}$-body only simulations (e.g. Kazantzidis et al. 2004; Bailin et al. 2005; Berentzen \& Shlosman 2006; Gustafsson et al. 2006; Abadi et al. 2010; Deason et al. 2011). However, since these studies used different sized haloes or simulations that had different feedback effects, it is difficult to make direct comparisons. For example Kazantzidis et al. (2004) ran Adaptive Refinement Tree $\mathrm{N}$-body + hydrodynamic simulations of cluster sized haloes and showed that the presence of baryons significantly increased the sphericity of the host dark matter haloes with a magnitude that decreased at larger radii such that the sphericity was largely indistinguishable from dark matter only haloes at the virial radius. Bailin et al. (2005) found very similar results, an increase in halo sphericity that decreases with increasing radius, using seven different implementations of hydrodynamics in the simulations and employing a multi-mass resimulation technique to generate high resolution galactic sized discs. Abadi et al. (2010) ran $N$-body + hydrodynamic simulations of galactic sized discs with radiative cooling but neglected stellar feedback. By calculating equipotential axial ratios they found haloes that were significantly rounder than their dark matter only counterparts at all radii out to nearly $2 R_{\text {vir }}$, which may be due to strong overcooling due to neglecting stellar feedback. By contrast, Deason et al. (2011) used simulations including stellar feedback and used the galaxy density to characterise the halo shapes (which are typically flatter than equipotential surfaces). The haloes in their sample were almost spherical and slightly more oblate in the inner regions, while their dark matter only haloes tended toward more prolate in the inner regions. Thus, they agreed that baryon physics significantly affects the shape of the halo in the inner regions but they did not see significant changes in sphericity in the outer regions between the $N$-body and hydrodynamic simulations.

Knebe et al. (2010) performed a similar analysis by comparing a dark matter only simulation with a hydrodynamic simulation with the same initial conditions. This study included 3 Milky Way sized galaxy haloes with their corresponding substructures. They tested the shapes of the dark matter haloes and subhaloes in their simulations and found the haloes to be more spherical in the hydrodynamic simulation, in agreement with the general consensus above. However, the subhaloes showed no shape differences between the two simulations, indicating that the addition of gas physics had no influence on the subhalo population. They suggested that the lack of shape changes may be due to the low mass nature of the subhaloes and that there may be more obvious changes for cluster sized host haloes with large galaxy sized subhaloes.

Bailin et al. (2005) tested whether the orientation of the inner halo was being driven by the galactic disc, by generating rings of test particles tilted by $10^{\circ}$ with respect to the disc plane and comparing the gravitational torque on the test particles due to the dark matter and the baryons. For test particles in rings $<0.1 R_{\mathrm{vir}}$, the baryons and dark matter exerted torques of comparable magnitude, while the dark matter dominated the torque at larger radii. Therefore, it was concluded that the alignments at inner radii were due to simultaneous evolution of the halo and disc, rather than the disc driving the halo orientation, or vice versa, and that the orientation of the inner and outer halo in the hydrodynamic simulations was uncorrelated. Tidal torque theory suggests that correlations between a galaxy and its host halo are expected during the formation epoch (e.g. Heavens \& Peacock 1988). However, while the alignment of a galaxy and its host halo is initially very close, at later times the outer halo will continue to accrete material. This could alter the net angular momentum or shape of the outer halo, causing the misalignments. This distinction is important when considering the alignment of satellite populations and their intrinsic alignment signal.

Thinking about how galaxy angular momentum aligns with its host halo, Bailin et al. (2005); Bett et al. (2010) and Deason et al. (2011) all showed that the alignment of the angular momentum vector of the galactic disc with the semi-minor axis of the host halo was close at $R<0.1 R_{\mathrm{vir}}$. Bailin et al. (2005) and Deason et al. (2011) also showed that the halo could be significantly misaligned at $R>0.1 R_{\mathrm{vir}}$ with misalignments of $\theta_{\lambda_{\mathrm{C}} \mathrm{H}}>45^{\circ}$ at $R_{\mathrm{vir}}$. 


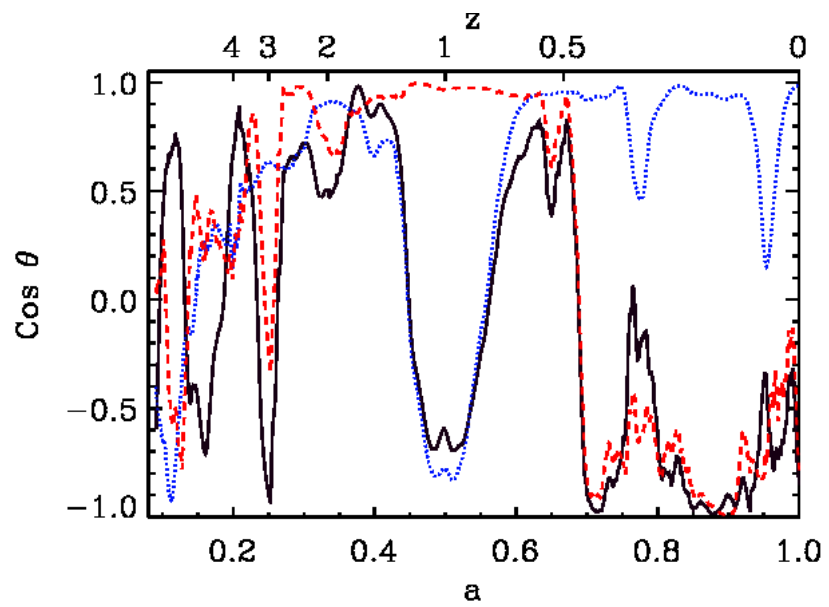

(a) Dark matter halo: $R_{\text {vir }}$, stars/gas: $8 \mathrm{kpc}$

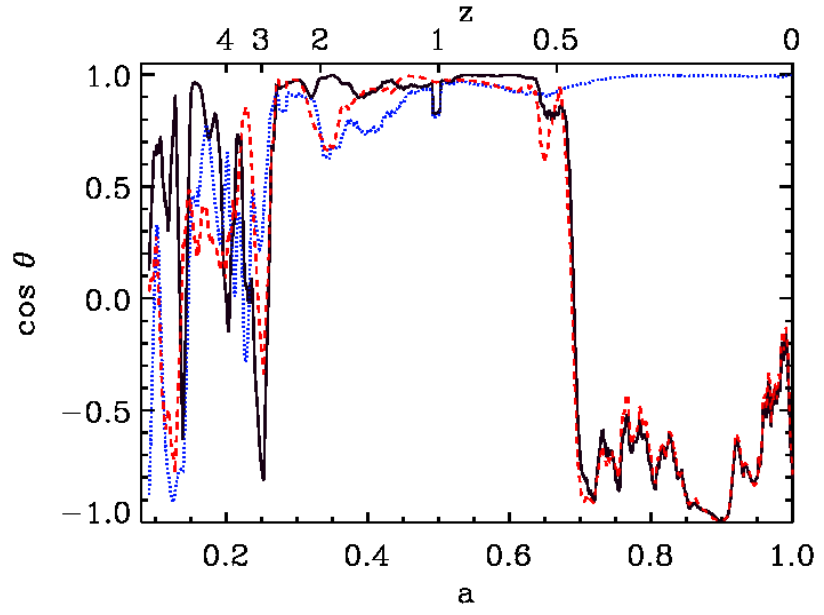

(b) Dark matter halo/stars/gas: $8 \mathrm{kpc}$

Fig. 13.- : Correlations between the angular momenta of the dark matter, gas, and stars of a $\sim 10^{12} h^{-1} \mathrm{M}_{\odot}$ galaxy system, within the radii given under each panel and as a function of scale factor/redshift . The angle $\theta$ is defined between the corresponding angular momenta, e.g. $\theta_{\lambda_{\mathrm{gas}} \lambda_{\mathrm{H}}}$. The colours represent the dark matter-gas (black, solid), dark matter-stars (blue, dotted) and stars-gas (red, dashed) correlations. (C) AAS. Reproduced with permission from Romano-Díaz et al. (2009).

van den Bosch et al. (2002); Chen et al. (2003); van den Bosch et al. (2003) and Sharma \& Steinmetz (2005) made measurements of the misalignments, $\theta_{\lambda_{\mathrm{C}} \lambda_{H}}$ at $R_{\mathrm{vir}}$ and found an average misalignment of between $20^{\circ}$ and $36^{\circ}$ for their standard baryonic simulations. In a similar study, Hahn et al. (2010) found that while the alignment of the angular momentum for both the central disc galaxy gas and stars was close to the angular momentum of the inner dark matter halo at $\theta_{\lambda_{\mathrm{c}} \lambda_{\mathrm{H}}} \sim 18^{\circ}$, there could be significant misalignments, $\theta_{\lambda_{\mathrm{c}} \lambda_{\mathrm{H}}} \sim 50^{\circ}$, with the angular momentum of the whole dark matter halo. This result appeared to have no dependence on environment density or stellar or halo mass.

There is a known problem in hydrodynamic simulations where cooling gas forming the central galaxies has an angular momentum that is too low to produce extended galaxies (e.g. Navarro \& Benz 1991). This may be partially due to numerical limitations, but uncertainties in the baryonic feedback processes are certain to contribute significantly (e.g. Maller \& Dekel 2002). While the feedback processes are being investigated separately, van den Bosch et al. (2003) tested the effects of artificially pre-heating the baryonic component of the intergalactic medium, and found that this pre-heating decoupled the baryons from the dark matter, resulting in significantly higher misalignments between the angular momentum of both the gas and the dark matter halo of $58^{\circ}$ on average.

Romano-Díaz et al. (2009); Scannapieco et al. (2009); Debattista et al. (2013); Cen (2014) and Debattista et al. (2015) investigated spin orientation changes over time. When investigating a $\sim 10^{12} h^{-1} \mathrm{M}_{\odot}$, somewhat isolated (no major mergers after $z=1.5$ ) central galaxy, Romano-Díaz et al. (2009) showed that the spin alignments of the dark matter halo (within $R_{\text {vir }} \sim 400 \mathrm{kpc}$ ), stars and gas (within the inner $8 \mathrm{kpc}$ ) changed frequently over time and even experienced $\sim 180^{\circ}$ flips over short timescales and at times with no major mergers (Figure 13a). When also limiting the dark matter to the inner $8 \mathrm{kpc}$, they found that at $z \gtrsim 3$ major merger activity caused all alignments to change frequently. From $3 \gtrsim z \gtrsim 0.5$, the spins of the dark matter, stars and gas were well aligned but at $z \lesssim 0.5$, the alignments flipped when cold gas became concentrated in the central regions and had a spin in the opposite direction to the stars and dark matter (Figure 13b). Scannapieco et al. (2009) found that misalignments between the stellar disc and newly accreted cold gas could cause the stellar disc to lose part of its mass or become completely destroyed (but later regrow).

Cen (2014) simulated and investigated spin alignments in more than 300 galaxies between $M_{*}=10^{10}-10^{12} \mathrm{M}_{\odot}$, They calculated the orientations of the specific angular momentum (Equation 40) for both gas and stars within the 
galaxy radius over time and showed that orientations were dependent on galaxy type (elliptical or spiral), environment, luminosity and redshift. They found that the spin alignments decreased with increasing redshift, increased with increasing stellar mass and were larger for elliptical than spiral galaxies.

It is clear from Cen (2014) that frequent changes in spin direction over time have a large effect on the final shape and orientation of a galactic disc. Mergers and cold gas accretion cause more stochastic and rapid orientation changes, while the large-scale tidal field slowly acts to bring galaxy spins back into alignment. However, as noted by Cen (2014), the question remains whether steady anisotropic infall of gas and stars along filaments and sheets could also act as a mechanism to align the spins of haloes along their structures.

\subsubsection{Satellite alignments}

The alignment of the distribution of satellite galaxies in host dark matter haloes, $\theta_{\mathrm{BH}}$, was extensively examined in Libeskind et al. (2007) and Deason et al. (2011). The method of analysis was similar in both studies although Libeskind et al. (2007) had 9 parent haloes (with only three of these having 11 or more satellites) and galaxies of all types were included in the analysis, while Deason et al. (2011) had 431 parent haloes (of which 80 had 10 or more satellites) and only late-type disc galaxies (the galaxies were classified dynamically as dispersion-supported spheroids or rotationally supported discs) were included in the analysis. Both computed the shape of the satellite distribution by diagonalising the unweighted inertia tensor (see Equation 33 and subsequent explanations). They both found that the distribution of ten or eleven brightest (most massive) satellites was significantly flatter (more planar) than the underlying dark matter distribution. They also found that satellite distribution preferentially aligned with the plane perpendicular to the semi-minor axis of the host dark matter halo. Deason et al. (2011) showed that accretion of satellites occurred preferentially along the semi-major axis of the dark matter halo and suggested that the anisotropic satellite distribution was due to anisotropic infall. This distribution was preserved because present day satellites had been in orbit for far less time than the dark matter halo, which would have undergone relaxation and phase mixing at an earlier time. Thus, the satellites had only ever experienced a static dark matter potential, which is reflected in the preserved anisotropic spatial distribution with the outer host halo. Deason et al. (2011) went on to show that the shape of the satellite distribution showed no preferential alignment relative to the galaxy, which is consistent with the alignment of the inner host halo and central galaxy being uncorrelated with the outer host halo where the satellite distribution is located (see Section 5.1.1). In fact, they found that $20 \%$ of their satellite distributions semi-major axes lay within $10^{\circ}$ of perpendicular to the plane of the galaxy, which is consistent with the observed distribution of the Milky Way satellites. Similarly, Libeskind et al. (2007) found two of their three galaxies with eleven or more satellites had the semi-major axis of the satellite distribution aligned within $20^{\circ}$ of perpendicular to the plane of the galaxy. Although with this study having only three galaxies, it is not possible to infer whether the satellite distribution showed any preferential alignments with the central galaxy.

In a separate analysis that accounted for all satellites in the systems, Deason et al. (2011) showed that the shape of the full satellite distribution continued to be much flatter than the shape of the dark matter halo, which is in contrast to Libeskind et al. (2007) who found that the full satellite distribution matched the shape of the dark matter halo well and the distribution did not exhibit the flattening found when only considering the eleven brightest satellites. However, it is important to remember that there were only three systems in the analysis by Libeskind et al. (2007), and all galaxy types were considered, making a direct comparison between the two studies difficult. Deason et al. (2011) also showed that the orientation of the satellite distribution shapes showed only a weak bias toward the plane of the galaxy but much stronger alignments relative to the plane perpendicular to the semi-minor axis of the host halo. The alignment of the satellite distribution was significantly more influenced by the dark matter host halo, so in the presence of misalignments between the inner and outer halo, the orientation of the galactic disc is largely decoupled from the satellites.

Knebe et al. (2010) investigated the alignment of subhalo semi-major axes with the centre of mass of the host dark matter halo, $\theta_{S h}$, in both a hydrodynamic and dark matter only simulation with the same initial conditions. Both simulations were a $2 h^{-1} \mathrm{Mpc}$ resimulated region in a $64 \mathrm{~h}^{-1} \mathrm{Mpc}$ parent simulation. They found that the subhaloes pointed preferentially toward the centre of the host halo in both simulations and that the addition of baryonic physics had no effect on the result. This is unsurprising given the subhaloes did not change shape with the addition of baryonic 
physics (as mentioned in Section 5.1.1).

\subsubsection{Large-scale structure Alignments}

There is some question of what the alignment of the semi-major axis of the baryonic components of galaxies is with respect to the large-scale structure. Navarro et al. (2004) used $N$-body/hydrodynamic simulations to produce four disc galaxies and investigated their alignments with sheets, $\theta_{\mathrm{GW}}$. They found that the semi-major axes of some disc galaxies tended to be highly inclined relative to the plane of the large-scale (several Mpc) two-dimensional sheet that the galaxy was formed in. This was likely due to coherence in anisotropies across a large number of scales present during the expansion and contraction of the protogalactic material causing some galactic discs to be highly inclined relative to the large-scale two-dimensional structure where they were embedded. Investigating alignments with respect to filaments, $\theta_{\mathrm{GF}}$, Hahn et al. (2010) sampled $\sim 100$ galactic discs spanning two orders of magnitude in stellar and halo mass. They found a strong alignment of the major axis of low mass $\left(<4 \times 10^{11} h^{-1} \mathbf{M}_{\odot}\right)$ galactic discs along the direction of the filament at $z=1$. This alignment decreased with increasing density, which may be due to non-linear effects like torques from ram pressure or accretion/mergers. They did not see this alignment at $z=0$, however this may be a numerical effect in their simulations. While admitting some statistical limitations in their studies, they also found that the most massive disc galaxies were aligned with their major axis pointing along the direction of a filament.

\subsection{Cosmological-volume hydrodynamic simulations}

When studying galaxy alignments, the use of hydrodynamic simulations over cosmological volumes is obviously highly desired. Large volume simulations enable a statistical approach to galaxy alignments by providing huge catalogues of galaxies. At the same time, if we want to use hydrodynamic simulations to predict intrinsic alignments on the scales that are relevant for lensing, up to tens of $\mathrm{Mpc}$, then the minimum simulation volume is $\sim 100 \mathrm{~h}^{-1} \mathrm{Mpc}$.

There are a number of recent papers on intrinsic alignments in hydrodynamic simulations in cosmological volumes, including Bryan et al. (2013); Tenneti et al. (2014, 2015a,b); Dubois et al. (2014); Welker et al. (2014) and Codis et al. (2015b). Bryan et al. (2013) used the OverWhelmingly Large Simulations (OWLS) (Schaye et al. 2010) to study the effects of baryonic cooling on the spins and alignments of dark matter haloes, rather than studying the baryonic components of the galaxies themselves.

\subsubsection{Alignments with spin and angular momentum}

Dubois et al. (2014); Welker et al. (2014) and Codis et al. (2015b) all focused on the issue of spin alignments using the Horizon-AGN simulation (Dubois et al. 2014) in a $100 \mathrm{~h}^{-1} \mathrm{Mpc}$ box. Using a catalogue of $\sim 150,000$ galaxies, Dubois et al. (2014) investigated a snapshot at $z=1.83$ and focused on the orientation of the spin of the galaxies, with respect to the direction of the filaments, $\theta_{\lambda_{\mathrm{G}} \mathrm{F}}$. They found that more massive galaxies have their spin perpendicular to the nearest filament while lower mass galaxies have their spin parallel to the nearest filament. The mass of this transition for the stellar disc was around $M_{*}=3 \times 10^{10} \mathrm{M}_{\odot}$, which is comparable to a dark matter halo transition mass at $z=1.83$ of $M_{\mathrm{h}}=5 \times 10^{11} \mathrm{M}_{\odot}$. This is consistent with the redshift evolution of the transition mass found in Codis et al. (2012) (see Section 4.2.5). The study went on to determine spin orientations with respect to the nearest filament for a large number of galaxy properties. The overall findings formed a picture that old, massive, red, metal-rich, and dispersion-dominated (elliptical) galaxies have their spins preferentially perpendicular to the nearest filament, while young, low-mass, blue, metal-poor, and centrifugally supported (disc) galaxies have their spins preferentially aligned parallel to the filament.

The Horizon-AGN simulation was run with an adaptive mesh refinement code that uses a Cartesian-based Poisson solver (Teyssier 2002) to compute the forces. A common issue in such a solver is forces grid-locking on the Cartesian axes of the box due to a numerical anisotropy in the force calculation (see e.g. Hockney \& Eastwood 1981). Dubois et al. (2014) found that gaseous disc spins of low-mass galaxies $\left(M_{\text {gas }} \sim 10^{9} h^{-1} \mathrm{M}_{\odot}\right)$ in the simulation were preferentially aligned with one of the Cartesian axes, while higher mass galaxies did not show any grid-locking. The cosmic web elements in the simulation did not experience any grid-locking so the overall effect of the grid-locked galaxies may make the measured alignments with the filaments more noisy. Codis et al. (2015b) presented evidence that if there is no spatial correlation between the grid-locking experienced by different galaxies, then the grid-locking would 
not significantly affect their results. However, a comparison of all of the grid-based solver studies with a solver that does not use a grid-based technique (like smoothed particle hydrodynamics (SPH) for example) would be required to test these assumptions and assess the full impact of the grid-locking on the results.

Welker et al. (2014) used the same simulations as Dubois et al. (2014) between $1.2<z<3.8$ in a study of the effect of mergers on galaxy spin changes. They showed that mergers along filaments caused galaxies to have a spin perpendicular to the filament and the more mergers that contributed to the galaxy mass, the stronger the perpendicular alignment. By contrast, in the absence of (successive) mergers, the galaxy spins would (re)align with the direction of the host filament.

Addressing the concerns of weak gravitational lensing, Codis et al. (2015b) used the spin of the stellar component of the galaxy as a proxy for ellipticity and investigated 160,000 simulated galaxies at $z=1.2$. When determining the apparent axis ratio for their projected galaxies, they ignored the disc thickness (which they set to zero) due to resolution limitations in the simulation, which has the effect of maximising the alignment signal measured and giving upper limits to the predictions. To investigate the GI (Equation 8) alignment signal, where correlations occur between the gravitationally sheared background galaxy and the intrinsically oriented foreground galaxy, they determined the galaxy population that was likely to experience coherent alignments as a result of the local tidal field. Similar to Dubois et al. (2014), they found that low-mass galaxies were preferentially aligned parallel to filaments and higher mass $\left(M_{*}>4 \times 10^{10}\right)$ galaxies were preferentially aligned perpendicular to the filaments. Additionally, red galaxies showed more random correlations with the surrounding tidal field than blue galaxies. Part of this may be related to the fact that red galaxies are typically more massive than blue galaxies and at $z=1.2$, the red galaxies in the simulation had a mass around the transition mass (some above, some below). The random orientations detected in this study might be expected to become more correlated at later times as more of the population exceeds the transition mass. However, it is also important to note that massive red galaxies are observed to be dispersion-dominated (see Kirk et al. 2015) and the spin of such a galaxy is not expected to be strongly correlated with its shape, meaning that the physical mechanism causing the observed alignments of massive red galaxies is unrelated to the spin. Further investigation would require use of the actual galaxy shapes rather than using spin as a proxy for the shape.

When investigating the II (Equation 8) alignment in 3D orientations, Codis et al. (2015b) found a strong correlation between the spins of blue galaxy pairs out to a distance of $10 h^{-1} \mathrm{Mpc}$, while red galaxy pairs and red-blue pairs showed no correlations. There was also a correlation of the spins between low- and intermediate-mass galaxies, while massive galaxies had a signal consistent with zero at all separations. These conclusions were unchanged when projecting the spin onto the plane of the sky.

Observational studies of spin alignments have shown some correlation between the spins of disc-like galaxies (e.g., Lee \& Erdogdu 2007; Lee 2011), but all were for $z<0.2$, while this Horizon-AGN simulation study was performed at $z=1.2$. Observations of shape alignments across a wide range of redshifts show red elliptical galaxies dominating the intrinsic alignment signal with blue disc-dominated galaxies being consistent with zero alignments (see Kirk et al. 2015, for a comprehensive overview of observational studies). At later times in the simulation, it will be interesting to directly compare the amplitudes of the signal with observations. For these to agree, it is possible that late-time effects that reduce the correlation of disc-dominated galaxy spins will be necessary. However, the alignments of dispersion-dominated red ellipticals would be better captured through study of the shapes of the galaxies directly.

\subsubsection{Alignments with shape and position}

Unfortunately, any reliable measurement of the shape of a distribution of particles requires the distribution to be sampled with a large number of particles. Convergence tests in, e.g., Tenneti et al. (2014) suggested that hundreds of (but preferably 1000) star particles are necessary to accurately infer galaxy shapes and therefore measure intrinsic alignments ${ }^{15}$ (with a similar requirement applying to the number of dark matter particles, when studying dark matter halo alignments). This leads to stringent requirements on resolution; for example, a study of intrinsic alignments of galaxies with stellar mass of $M_{*} \geq 10^{10} \mathrm{M}_{\odot}$ requires a star particle mass of $10^{7} h^{-1} \mathrm{M}_{\odot}$. Relevant to the understanding

${ }^{15}$ There are measurements in the literature that use as few as 20 particles. The results in this paper suggest that those results should be approached with caution. 

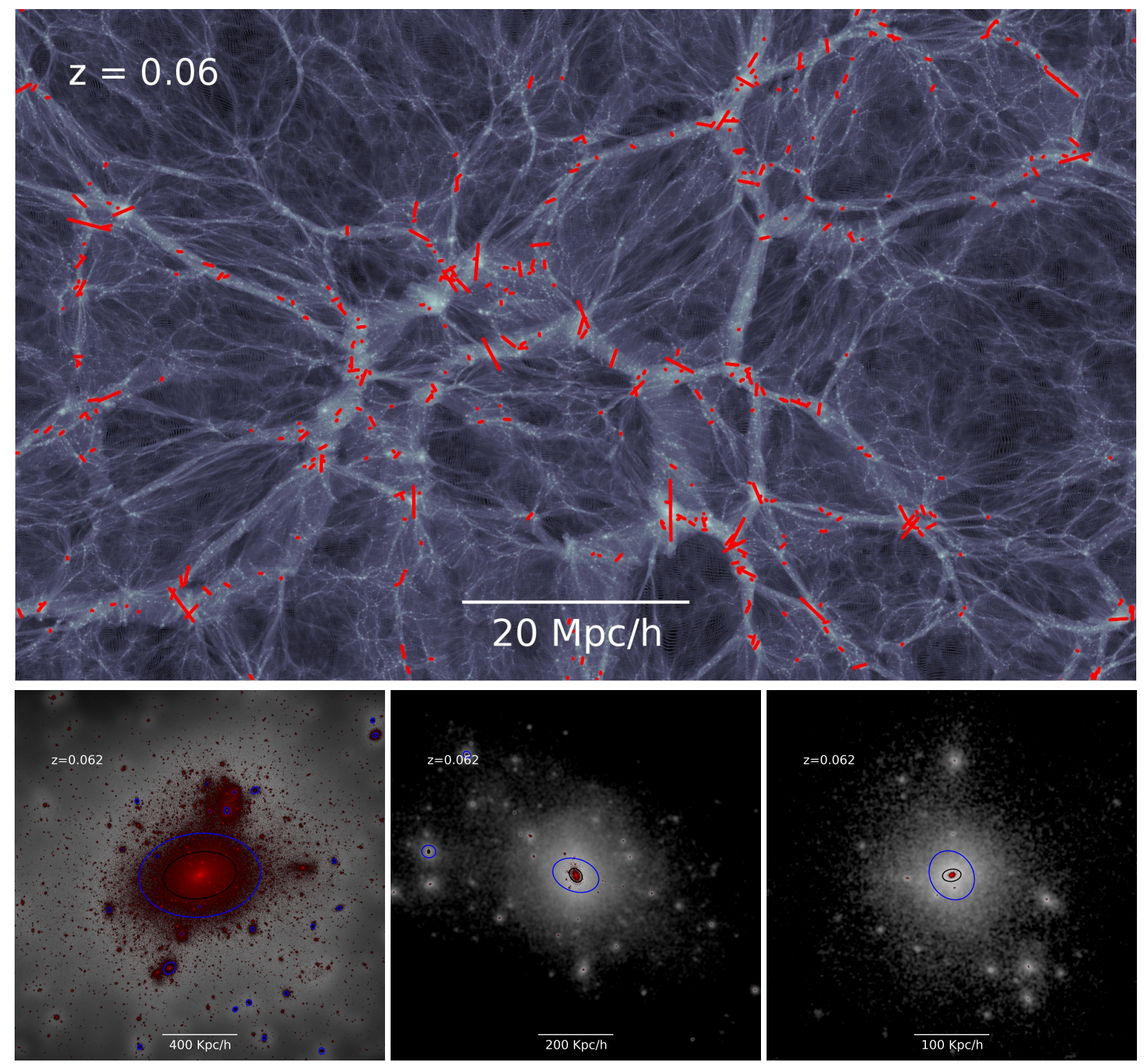

Fig. 14.—: Top: Snapshot of the MB-II SPH simulation in a slice of thickness $2 h^{-1} \mathrm{Mpc}$ at redshift $z=0.06$. The bluish-white colours represent the density of the dark matter distribution and the red lines show the direction of the major axis of ellipse for the projected shape defined by the stellar component. The length of the lines is linearly proportional to the semi-major axis length. Bottom Left: Dark matter (shown in grey) and stellar matter (shown in red) distribution in the most massive group at $z=0.06$ of mass $7.2 \times 10^{14} h^{-1} M_{\odot}$. The blue and black ellipses show the projected shapes of dark matter and stellar matter of subhaloes respectively. Bottom Middle: Dark matter and stellar matter distribution in a group of mass $3.8 \times 10^{12} h^{-1} M_{\odot}$. Bottom Right: Dark matter and stellar matter distribution in a group of mass $1.1 \times 10^{12} h^{-1} M_{\odot}$. Reproduced with permission from Tenneti et al. (2014).

of intrinsic alignments is the study of the shapes and alignments of galaxies with respect to the (mostly dark) matter distribution in their host haloes. In this case, a stochastic misalignment, $\theta_{\mathrm{GH}}$ (see Table 1), between galaxies and their 
host haloes suppresses the galaxy intrinsic alignments with respect to those from $N$-body simulations.

Tenneti et al. (2014) used the MassiveBlack-II SPH simulation (MB-II; Khandai et al. 2015), with star formation and AGN feedback, to study the shapes and alignments of galaxies with >1000 particles (Figure 14). In a similar study, Velliscig et al. (2015) used the Evolution and Assembly of GaLaxies and their Environments (EAGLE; Schaye et al. 2015; Crain et al. 2015) and cosmo-OverWhelmingly Large Simulations ( cosmo-OWLS; Schaye et al. 2010; Le Brun et al. 2014; McCarthy et al. 2014) to study the alignment and shape of dark matter, stellar, and hot gas distributions with $>300$ particles. Both investigations found that misalignments, $\theta_{\mathrm{GH}}$, were larger for lower-mass haloes than for higher-mass haloes and Tenneti et al. (2014) showed that the misalignments were only weakly dependent on redshift (see Figure 15a). Velliscig et al. (2015) showed that, in the EAGLE simulation, the median misalignment angle was reduced to $\sim 25-30^{\circ}$ in the most massive haloes $\left(10^{13} \leq M_{200} /\left[h^{-1} \mathrm{M}_{\odot}\right] \leq 10^{15}\right)$ and both Velliscig et al. (2015) and Tenneti et al. (2014) showed that the misalignment angles were systematically reduced at all masses when considering 2D, rather than 3D, misalignment angles. Some observational studies (e.g. Heymans et al. 2004; Okumura et al. 2009) have assumed a Gaussian functional form for the distribution of the 2D stochastic misalignment angle between galaxies and their host haloes. However, both Tenneti et al. (2014) and Velliscig et al. (2015) showed that the probability distributions of the misalignment angle were not Gaussian (Figure 15). Figure 15b shows an example of a non-Gaussian distribution of misalignment angles for dark matter haloes, which are potential hosts of Luminous Red Galaxies (LRG), from Velliscig et al. (2015) compared with the assumed Gaussian functional forms from Okumura et al. (2009). These results show the importance of exercising caution when employing oversimplified assumptions about the stochastic misalignment between galaxies and haloes and when interpreting the measurements of galaxy intrinsic alignment correlation functions.

Velliscig et al. (2015) calculated the misalignment of the baryonic components (hot gas and stars) of the galaxies with their host haloes as a function of halo mass, radius, and galaxy type. Overall, galaxies were found to align well with the inner host haloes. However, the stellar distributions exhibited a median misalignment of $\sim 45-50^{\circ}$ with respect to the outer host haloes. One simulation, calibrated to reproduce the stellar mass function at redshift zero, was used for comparison with the simulations that were generated using different feedback models. Importantly, the simulations that did not reproduce the basic properties of galaxy populations, such as abundance (number density per comoving volume) and galaxy size, exhibited differences in the median misalignment angle between galaxies and their host haloes of the order of $10^{\circ}$ and this could be as large as $20^{\circ}$ for the case where AGN feedback was neglected in the simulation.

Both Tenneti et al. (2014) and Velliscig et al. (2015) used only the unweighted inertia tensor defined in Section 4.1.2. However, a subsequent study by Tenneti et al. (2015a) noted that this might have an impact on the conclusions, as shape parameters and alignment angles significantly depend on the way they are computed. For instance, Tenneti et al. (2015a) included a study of how the results depended on the use of unweighted or reduced inertia tensors; the latter weighting more the inner regions of galaxies. However, weighting star particles by luminosity rather than stellar mass did not lead to a noticeable change.

Tenneti et al. (2015a) took the work in Tenneti et al. (2014) a step further and measured the intrinsic alignment 2-point correlation functions in the MB-II simulation. These included measurements of position-angle statistics and projected correlation functions like $w_{g+}$ (Equation 7) for galaxies as a function of mass, luminosity, and stellar mass, with tabulated fits to the non-linear alignment model (see Section 3.3) that demonstrate the scalings with these parameters. A preliminary study was made of colour-dependence, with blue galaxies having lower intrinsic alignment amplitudes than red galaxies; however, the colours of the galaxies were not fully realistic, so a more detailed study would be required to understand these results. Comparison of $w_{g+}$ for central and satellite galaxies within fixed subhalo mass ranges suggests that at lower mass, central and satellite galaxies have similar small-scale alignments $\left(<1 h^{-1} \mathrm{Mpc}\right)$ but satellites have smaller (but non-zero) large-scale alignments. At intermediate subhalo masses, $10^{11.5}<M<10^{13} h^{-1} \mathrm{M}_{\odot}$, central and satellite subhaloes were found to have similar intrinsic alignments on all scales, which is somewhat in tension with observations (see Kirk et al. 2015). The $w_{g+}$ predictions for a massive galaxy sample were reasonably consistent with SDSS luminous red galaxy measurements, which provided some validation that the simulation included the relevant processes for galaxy intrinsic alignments of at least massive galaxies.

More recently, Tenneti et al. (2015b) investigated a critical ingredient of the previous results: the impact of galaxy 


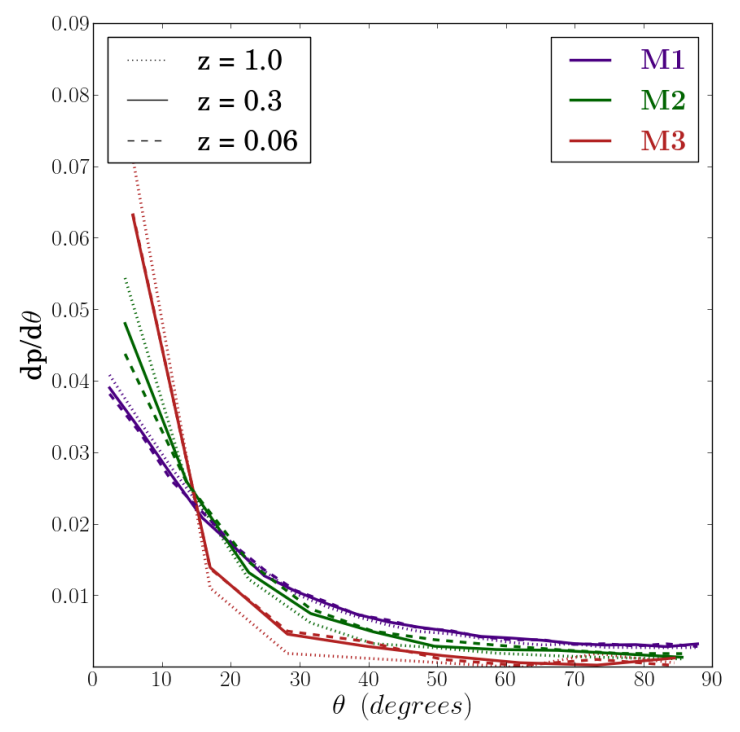

(a) MassiveBlack II

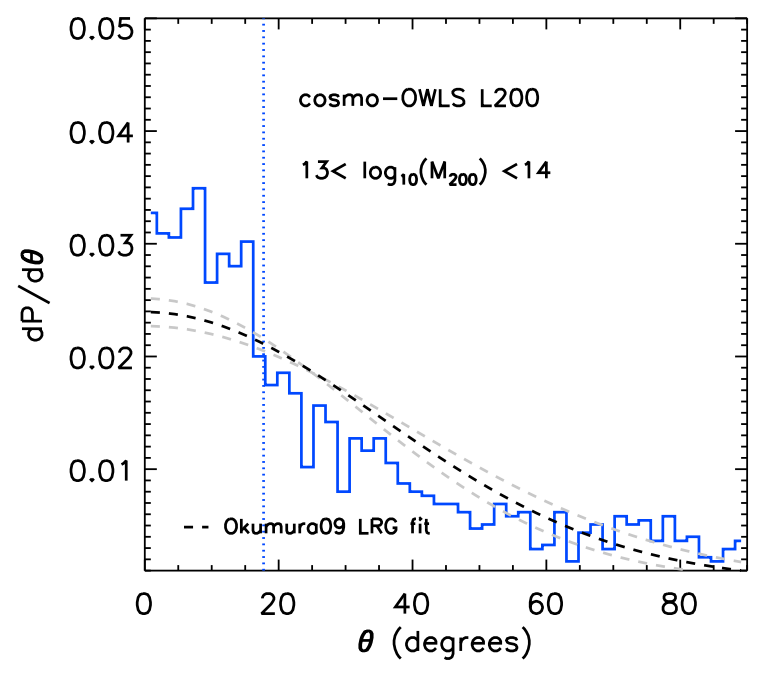

(b) cosmo-OWLS

Fig. 15.- : Left: Histogram of 2D misalignments from MassiveBlack II between galaxy and dark matter halo shapes, $\theta_{\mathrm{GH}}$, at redshifts $z=1.0,0.3$, and 0.06 in mass bins $M 1, M 2$ and $M 3$ that go from lower to higher mass (see Tenneti et al. 2014 for details). The $M 3$ mass bin is the most similar to the mass bin used in the right panel of this figure. Reproduced with permission from Tenneti et al. (2014). Right: Histogram of 2D misalignments from cosmo-OWLS between stars (inside the stellar half-mass radius) and the total matter distribution for haloes in the mass bin $10^{13} \leq$ $M_{200} /\left[h^{-1} \mathrm{M}_{\odot}\right] \leq 10^{14}$ and at $z=0$, reproduced from Velliscig et al. (2015). The vertical line indicates the median value of the misalignment angle. The black (mean) and grey (one sigma deviation) curves are the analytic Gaussian functional forms that were employed in the Okumura et al. (2009) observational study of Luminous Red Galaxies (LRG). Figure Credit: Marco Velliscig.

formation physics on intrinsic alignments. This work relied on direct comparison between MBII and a dark matteronly simulation with the same box size, cosmology, resolution, and initial conditions, so that any differences in the results are due only to the additional physics in the hydrodynamic simulations. The study matched dark matter haloes between the two simulations and showed that the shapes of the matched full dark matter haloes had similar orientations, especially at the highest halo masses. They also measured the alignment of the stellar component in MBII with that of the inner part of the dark matter halo in the two simulations. A correlation between the orientations was found in either case. However, the correlation between the orientation of the stellar component in MBII and the matching halo in the dark matter-only simulation was weaker than that between the stellar component in MBII and the halo in MBII. Further, the authors showed that misalignments between the stellar component and the dark matter halo increased with increasing radii in MBII, while the misalignment of the stellar component in MBII with the dark matter-only halo showed no significant change with increasing radius. This indicates that there is some coevolution of the baryons and the dark matter at the inner halo radii, which is similar to the results of Bailin et al. (2005) (see Section 5.1.1). However, unlike Bailin et al. (2005), Tenneti et al. (2015b) did find a weak correlation between the MBII inner and outer haloes that may have been missed in this earlier work (and also in the satellite alignment study of Deason et al. 2011, see Section 5.1.2) due to the smaller number statistics. Tenneti et al. (2015b) concluded that it would not be useful to measure the shape of the dark matter component at the inner radii as a means to trace the shape and orientation of the stellar component and that a mapping between the hydrodynamic and dark matter-only simulations should utilize the shapes of the full haloes. For more detail on those results, see Tenneti et al. (2015b).

Dong et al. (2014) investigated the alignments of the satellite distribution using the positions of satellites within their host halos. An SPH simulation was used with a $100 \mathrm{~h}^{-1} \mathrm{Mpc}$ box, including the effects of gas cooling, star formation, 
and stellar feedback (although no AGN feedback, which is necessary to avoid over-cooling). They investigated the alignment of central galaxies with the positions of satellites, finding stronger alignments both for red centrals and red satellites, consistent with the observational picture (see Kirk et al. 2015 for more details). Interestingly, they found a physical reason for this effect, which was that the red satellites stayed closer to the inner regions of the dark matter halo, which correlated strongly with the central galaxy shape (unlike the outer regions of the dark matter halo, which were less correlated with the central galaxy shape). They found trends with the host halo mass and with satellite galaxy metallicity, providing predictions that could be tested with future datasets.

\subsection{Hydrodynamic simulations Roundup}

In this section, small-volume and zoom hydrodynamic simulations were reviewed as well as large cosmologicalvolume hydrodynamic simulations. There is far less literature in this area as hydrodynamic simulations are time consuming and the baryonic processes that occur on scales below the resolution of the simulation are not well understood. Small-volume simulations can be run reasonably quickly, but suffer from small number statistics. Cosmologicalvolume simulations contain a good statistical sample of galaxies but are more difficult and time consuming to run, meaning that there are currently very few cosmological-volume simulations suitable for galaxy alignment studies.

It is clear that the addition of baryons cause the shapes of the dark matter haloes to change. Haloes with baryons tend toward oblate, rather than the prolate shapes found in the $N$-body simulations. There is a discernible correlation between the galaxy and the inner halo, which is the result of coevolution of the baryons and the inner dark matter halo, but the addition of baryons causes the alignment of the inner and outer haloes to become only weakly correlated.

Satellite distributions appear to be well aligned with the outer host halo when late-type disc galaxies are considered, while red early-type galaxies tend to be better aligned with red central galaxies. It appears that red satellites may be generally located closer to the centre of the halo, meaning that they would be more subject to the tidal forces of the inner halo.

The relative alignments of the dark matter, gas and star spin vectors within a galaxy system may experience numerous flips over its lifetime, which will have a significant effect on the shape and orientation of the galaxy over this time. From a galaxy formation and evolution perspective, this is certainly an interesting field to pursue. However, it is important to note that while spin alignments are likely the dominant alignment mechanism for rotationally-supported galaxies, the study of spin alignments may omit some critical details for intrinsic alignment contamination of weak lensing measurements, given that observed intrinsic alignments are dominated by red galaxies, which are pressuresupported and thus subject to intrinsic alignments due to different physics (e.g., tidal effects).

There is some limited (to date) but compelling evidence that galaxy alignments have some dependence on the sub-grid physics, within our current abilities to determine this given the small number of hydrodynamic simulations with sufficient volume and resolution to answer this question. Further studies on this topic would be useful. It is, however, clear that the future of galaxy intrinsic alignment studies will certainly benefit from extensive research using hydrodynamic simulations for progress and this is discussed further in Section 7.

\section{Semi-analytic modelling}

It will remain impossible for the foreseeable future to run hydrodynamic simulations with the volumes, resolutions, and number of realisations required to generate the high precision covariance matrices required for upcoming telescope missions, or for direct, robust and precise, measurements of intrinsic alignments. An established way to link the output of pure $N$-body simulations to observables of galaxies is the use of analytic prescriptions that take the properties of dark matter haloes as input. They can be informed by simplified and parametrised physical models or by effective relations determined from higher resolution or hydrodynamic simulations.

Such semi-analytic models of galaxy formation and evolution are routinely used to predict observables like galaxy clustering and luminosity functions (see Bower et al. 2006 for a review). These semi-analytic models of galaxies have been embedded in a number of $N$-body simulations in order to investigate weak lensing II and GI signals (e.g. Okumura et al. 2009; Okumura \& Jing 2009) and satellite alignments in haloes (e.g. Zentner et al. 2005; Kang et al. 2005; Agustsson \& Brainerd 2006, 2010; Kang et al. 2007; Metz et al. 2008; Bailin et al. 2008; Faltenbacher et al. 
2009; Libeskind et al. 2009) ${ }^{16}$. The requirement for realistic galaxies lies in the fact that observations have indicated that satellite alignments appear to be different for a range of galaxy types; e.g. early- and late-type host galaxies, red and blue host galaxies, satellites with low and high star formation rates (see Kirk et al. 2015, for further discussion on observational satellite alignment results). If the central galaxy is naively assumed to follow the shape of the host halo, the semi-analytic model predicts a larger alignment signal than the observations (e.g. Agustsson \& Brainerd 2006; Kang et al. 2007). It is also interesting to note that, in order to correctly reproduce magnitudes and colours, the models have to take into account a notion of size such as the scale length of discs. Therefore it seems plausible to extend the semi-analytic approach to the shapes and orientations of galaxies.

The simplest ansatz assumes that galaxies are homologous with the underlying dark matter halo (in 3D or in projection), which may be applicable to elliptical galaxies. In their simulation analysis, Heavens et al. (2000) proposed additionally a model for spiral galaxies, which are assumed to be thin discs perpendicular to the angular momentum of the host dark matter halo. Heymans et al. (2004) extended the spiral model in two ways: (a) they now assumed a thick disc via rescaling all ellipticities by a constant factor 0.73 which was estimated from observed ellipticity distributions, and (b) they allowed for a misalignment between the angular momentum of the galaxy and the halo, randomly drawn from a distribution fitted to the results from the simulations of van den Bosch et al. (2002).

Heymans et al. (2006) made the first attempt to model a realistic galaxy population with intrinsic alignments based on the conditional luminosity function by Cooray \& Milosavljević (2005), which provides the halo occupation with elliptical or spiral galaxies of a certain luminosity for a given redshift and halo mass. Using only haloes with occupancy one, i.e. central galaxies, Heymans et al. (2006) assigned elliptical galaxies the shape of the parent halo and modelled spiral galaxies as in Heymans et al. (2004). The resulting 'mix' model of elliptical and spiral galaxies produced an II signal that is in good agreement with the SDSS main sample measurements of Mandelbaum et al. (2006b). Heymans et al. (2006) also found a clear GI signal that showed no redshift evolution and increased with host halo mass.

While the halo occupation formalism captures the average alignment properties of galaxies with a certain halo mass, a full semi-analytic model is capable of tracking any dependencies on the merger history of an individual halo. Thus, only the latter can account for correlations between alignment and galaxy luminosity, colour, morphological type, etc. induced by the evolution of that galaxy. Joachimi et al. (2013a,b) complemented the Millennium Simulation and the Durham semi-analytic models (Bower et al. 2006) with a range of prescriptions for galaxy shapes.

Elliptical galaxies were still assumed to follow the shape of their haloes; however, while the aforementioned works did not specify how the halo ellipticity was measured, Joachimi et al. (2013a,b) considered explicitly an ellipticity based on the simple inertia tensor (see Equation 33 and following text) and a rescaled version approximating the reduced inertia tensor, the latter generally producing more circular galaxy shapes. Spiral galaxies were modelled again as a thick disc, but the misalignment distribution was updated to one from Bett (2012) and the disc was approximated as an opaque cylinder with two different values of thickness. Satellites were included, but as no subhalo properties were available, their shapes were assumed to be determined by the same prescription as the respective central galaxy type, in some cases with rescalings based on the observations by Knebe et al. (2008a). All satellites point towards the centre of the host, with misalignment distributions taken from Knebe et al. (2008a) and Bett (2012).

These models were confronted with intrinsic ellipticity distributions for various galaxy samples extracted from the COSMOS survey, as well as the GI correlation measurements of Joachimi et al. (2011) and Mandelbaum et al. (2011). No combination of models was able to fit all observables simultaneously, although the semi-analytic predictions generally reproduced at least the correct trend and order of magnitude (see Figure 16), despite the numerous simplistic assumptions. A notable exception is the GI correlation function $w_{\delta+}$ for early-type galaxies with $M_{r}<-19$ for which all models strongly over-predict the amplitude. This could indicate a failure of the alignment or galaxy evolution models for satellite galaxies, which dominate these samples, or suggest that the linear luminosity scaling of $w_{\delta+}$ found by Joachimi et al. (2011) cannot be extrapolated to fainter magnitudes.

There are clear avenues for improving semi-analytic models of galaxy shapes and orientations, such as updating scaling and alignment relations from state-of-the-art simulations (e.g. Tenneti et al. 2014) and iterating on the alignment mechanisms, e.g. by including information on the local tidal shear tensor instead of just halo shape. The

${ }^{16}$ Some of these studies use a halo occupation distribution (HOD; e.g. Berlind \& Weinberg 2002) approach to reproduce observed galaxy properties. This is a more statistical approach to matching galaxy properties than semi-analytic modelling. 


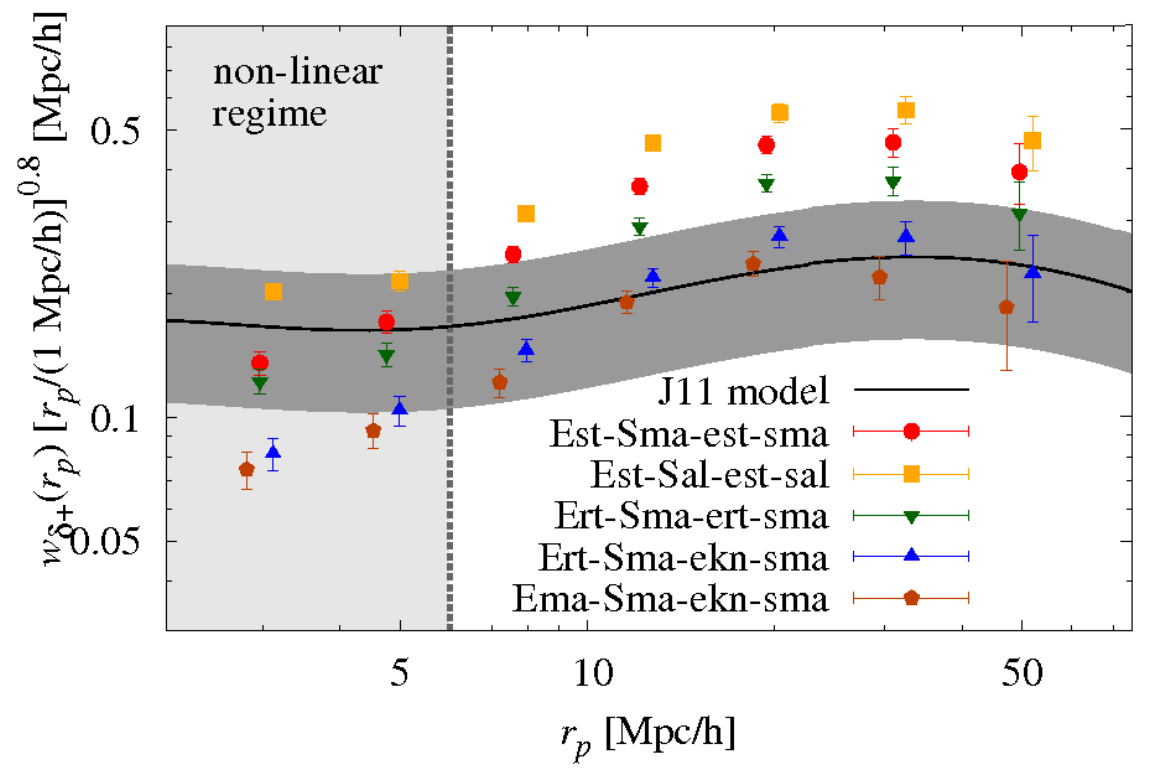

Fig. 16.- : Correlation function between matter density and tangential galaxy ellipticities, $w_{\delta+}$, as a function of comoving transverse separation $r_{p}$. The signals resulting from various parametrisation choices in the semi-analytic prescription are shown as coloured symbols (for a detailed explanation see Table 1 of Joachimi et al. 2013b). Error bars are determined from the variance between 64 simulated fields. The black line is the prediction by the Joachimi et al. (2011) form of the non-linear alignment model, using their best-fit parameters for the amplitude and the luminosity and redshift dependencies. The dark grey band marks the uncertainty propagated from the $1 \sigma$ errors on these parameters. Signals from the mocks have been divided by linear galaxy bias, so that the measurements below $6 h^{-1} \mathrm{Mpc}$ cannot directly be compared to the prediction. Reproduced with permission from Joachimi et al. (2013b).

requirements on the underlying $N$-body simulations are quite demanding, especially on the mass resolution, which should at least allow for about 300 particles (ideally more than $10^{3}$ particles) per halo for which shapes and angular momenta are to be measured (Bett et al. 2007; Joachimi et al. 2013a; Tenneti et al. 2014). If these halo properties are determined only for the central part of haloes, then the same requirement will translate into even lower particle mass for the simulation. For sufficiently small scales and high precision the impact of baryons on the properties of the dark matter halo will become relevant, making an analytic link between luminous and dark matter alignments even more challenging.

\section{Roadmap/wish list}

When planning to connect theoretical predictions and observations, what would an idealized roadmap or wish list look like?

In an ideal scenario with unlimited resources, simulations would be run in a large $(\simeq 4 \mathrm{Gpc})$ box with a very high particle mass resolution ${ }^{17}\left(\simeq 10^{6} \mathrm{M}_{\odot}\right)$. Suites of hydrodynamic simulations would be generated using many different simulation codes for comparison and a wide exploration of baryonic physics and feedback effects would be included to gauge their impact on the simulations and to compare with observations for accuracy. In reality, these simulations would be impossible to run, using far more CPU hours, memory, disk space and time than are available in the world today. Given a realistic landscape, the following list represents tasks that would be useful to pursue for future galaxy

\footnotetext{
${ }^{17}$ The large box size would account for the large scales that should be accounted for in studies of the large-scale structure and the high mass resolution would resolve the shapes and details of individual galaxies with high precision.
} 


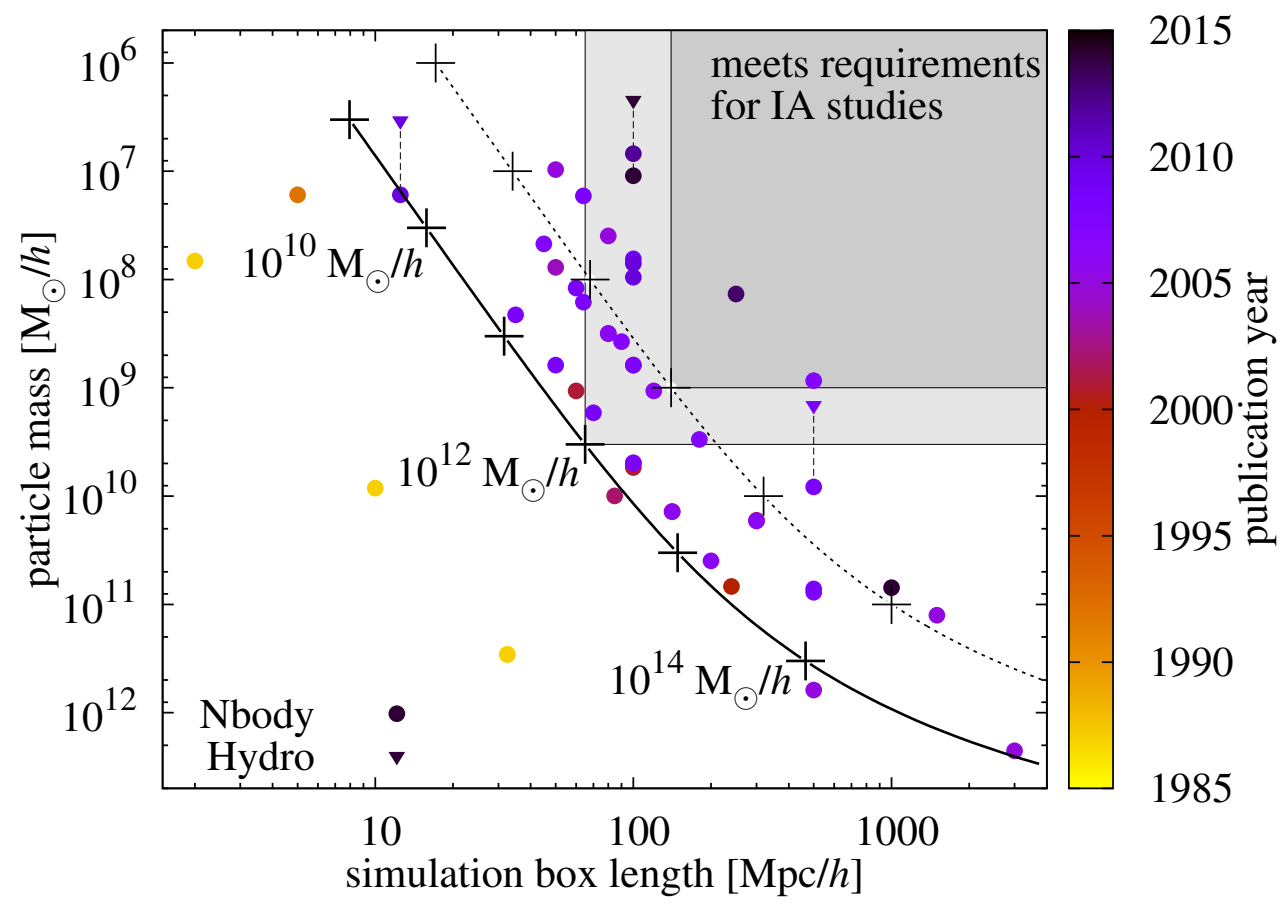

Fig. 17.- : Simulation specifications critical for halo/galaxy alignment studies. Plotted are the particle mass and simulation box length for simulations underlying a selection of alignment works, with the publication year of said works given by the colour coding. Dots (triangles) represent $N$-body (the gas/stellar component of hydrodynamic) simulations. Dashed lines link the gas and dark matter parts of a given simulation. The black solid (dotted) line connects points at which one expects to find $10^{3}\left(10^{4}\right)$ haloes of a given mass that are sampled with 300 (1000) particles each. Multiples of ten for these halo masses are indicated by the crosses. Areas to the top and right of a given point on the lines cover simulations that fulfill the minimum (solid) or strict (dotted) criteria for accurate alignment measurements of haloes of that mass, as illustrated by the grey areas for a Milky Way sized halo $\left(10^{12} h^{-1} \mathrm{M}_{\odot}\right)$.

alignment analyses.

\subsection{Simulations with sufficient resolution and numbers of haloes}

Simulations are currently still the best way to test intrinsic alignment models and mitigation techniques. The first task on this wish list is to determine the size and resolution that simulations should have for future intrinsic alignment studies. When investigating the properties of $N$-body haloes, Bett et al. (2007) found that in order to mitigate numerical artifacts, each halo required greater than 300 particles. However, a more recent study on hydrodynamic simulations found that results converged when haloes had 1000 particles (Tenneti et al. 2014). A simulation with $10^{3}$ haloes resolved will provide a minimum statistical sample, while $10^{4}$ haloes will generate a more statistically significant result. These numbers are roughly informed by the galaxy sample sizes of observational intrinsic alignment studies (e.g. Joachimi et al. 2011), where a few thousand galaxies are typically required for a detection, while a few ten-thousand galaxies allow for the definition of subsamples and higher signal-to-noise. Figure 17 shows a census of published simulations that have been used for halo/galaxy alignment studies to date. Only simulations with a fixed resolution, to allow for a direct and fair comparison, are included (zoom simulations and resimulations are excluded). The area above and to the right of the solid (dotted) line is where a simulation would need to lie to meet the minimum (strict) criteria for performing galaxy alignment studies of galaxies in haloes of a given mass. The example shown in this figure is for studies wishing to resolve Milky Way sized haloes $\left(10^{12} h^{-1} \mathrm{M}_{\odot}\right)$ and currently only two of 
the simulations published meet the strict criteria required for intrinsic alignment studies. Therefore, when planning simulations for future intrinsic alignment studies, this figure can be used to determine minimum box size and particle resolution requirements to ensure that the simulations produce meaningful results. Needless to say, the simulations described in the following tasks in this wish list should all conform to the requirements outlined here.

\subsection{A mapping between hydrodynamic and dark matter-only simulations}

The addition of baryons to a simulation has an impact on the dark matter distribution, as was shown in Section 5. In order to quantify these effects, the second task in this wish list is to provide a mapping between hydrodynamic and dark matter-only simulations. This will require a detailed comparison between a hydrodynamic simulation and a dark matter-only simulation with exactly the same initial conditions. The resulting simulations should contain the same dark matter haloes in general ${ }^{18}$, which will enable a quantative comparison. Tenneti et al. (2015b) performed an initial analysis of dark matter halo and subhalo shape and orientation misalignments, as a function of radius from the halo centre, between two such simulations. They showed that the shapes and orientations measured between the simulations was far from random and that it should be possible to develop a mapping between the simulations. Further investigation is still required to quantify such a mapping and then to utilize this to produce more precise semi-analytic models.

\subsection{Accurate galaxy properties in hydrodynamic simulations}

Beyond the technicalities involved in the estimation of galaxy shapes and misalignments in Sections 4 and 5, the open challenge in studying galaxy alignments with hydrodynamic simulations remains the fact that the same processes that determine galaxy shapes and mutual alignments are connected (if not exactly the same) to those that give rise to several other observed scaling relations. Unfortunately, hydrodynamic simulations have not yet converged on an implementation of the physics behind these processes and often they fail in reproducing basic observables such as the number density of galaxies as a function of their luminosity and/or the mass-size relation. Although these simulations are ideal tools to highlight possible hidden connections between different processes, they have not yet reached the maturity to have predictive power. The third task in this wish list is the most challenging and time consuming - to produce hydrodynamic simulations with more realistic galaxy properties.

For galaxy alignment studies, there is good evidence to suggest that the alignment signal is a function of halo mass (e.g. Schneider et al. 2012), but observations provide luminosity or stellar mass. However, see Singh et al. (2015) who investigated galaxy intrinsic alignments as a function of halo mass as determined from simultaneous galaxy weak lensing measurements. On the largest scales, theory appears to match the observed galaxy alignments reasonably well (Section 3.1; Figure 8) and they appear to be well modelled phenomenologically on the smallest scales (Section 3.2; Figure 8). Therefore, if hydrodynamic simulations are able to match the observed stellar and halo masses, matching the galaxy alignment signal to observations should be possible. However, modelling of the intermediate scales is still largely unknown and poorly constrained (Section 3.3) and the transition from the linear regime to the non-linear (high density) regime is where simulations will provide significant insight to the understanding of galaxy alignments (see Figure 12 in Joachimi et al. 2015). It is also clear that the environment (local and large-scale) should have an impact on intrinsic alignments. As an initial request, the simulation should match the abundances of galaxies as a function of stellar mass, type (early- and late-type) and colour. As an additional step, it would be desirable to match the size and ellipticity distributions ${ }^{19}$ of galaxies to observations.

\subsection{High precision semi-analytic simulations}

The hydrodynamic simulations outlined in Section 7.3 will be essential to providing insight into how galaxies form and evolve and in modelling the galaxy alignment signal. However, generating these simulations will use enormous

\footnotetext{
${ }^{18}$ Some differences between the hydrodynamic and dark matter-only simulation haloes should be expected and may arise if the halo finders split a structure into different haloes or some haloes fall below the minimum particle threshold between the realisations etc.

${ }^{19}$ In order to match ellipticity distributions, hydrodynamic simulations would need to resolve the thickness of the discs accurately, which would require resolving interstellar medium processes accurately. It is not clear that this final point will be possible in the simulations, so this task may have to remain on the "wish" list.
} 
computing resources - both CPU hours and disk space - so it will not be possible to generate the large suites of simulations that are required for upcoming weak gravitational lensing surveys. Therefore, a faster way to generate simulations that contain realistic intrinsic alignments is required. The final task in this wish list is to take the galaxy alignments and galaxy properties derived from these increasingly realistic hydrodynamic simulations in Section 7.3 and use the mapping identified in Section 7.2 to populate $N$-body simulations. The semi-analytic simulations in Section 6 used theoretical models of alignments to populate the $N$-body simulations. This task represents the next generation of semi-analytic simulation that contains the increasingly realistic galaxy properties and alignments derived from hydrodynamic simulations, likely in the form of a probability distribution to account for the generally stochastic nature of the processes involved. Another option to populate the $N$-body simulations may be to use machine learning algorithms that use hydrodynamic simulations or, use current observations of intrinsic galaxy alignments, as a training set. This task should be completed concurrently with the previous tasks. For each advance in knowledge coming from hydrodynamic simulations, many $N$-body simulations may be populated for testing and development of mitigation techniques.

\subsection{Moving Forward}

The efforts outlined here represent areas where significant progress can be made in understanding galaxy alignments. However, simulations alone will not be able to provide a complete understanding. Additional progress will be made by combining simulations with observations and analytic modelling, especially in the transitions between the linear and non-linear regimes. Hydrodynamic and semi-analytic simulations must be compared with observations to ensure accurate reproduction of galaxy properties. Observations also provide constraints on galaxy alignments directly, which can then be compared with the simulations and used to inform the analytic models. Similarly, insights coming from the simulations can be used to inform the analytic models with the hopes of unifying the models over all scales (see Section 3.4).

These tasks are time consuming, challenging and in some cases may not be possible (particularly some of the tasks in Section 7.3), but these are areas where efforts should be focused to ensure continued progress in understanding how intrinsic alignments form and evolve.

\section{Concluding Remarks}

While the title of this review refers to galaxies, as they are the primary observable accessible to large-scale structure observations, this review has shown that, within a theoretical and simulation perspective, it is possible to measure the shapes and orientations of structures over a wide range of scales, from satellite galaxies and subhaloes through to the large-scale structures of the cosmic web.

Although a reasonably consistent picture exists for alignments of dark matter haloes in $N$-body simulations, these are proving to be of limited use in understanding the alignments of galaxies, which remain the focus of interest for both galaxy formation and evolution and weak gravitational lensing studies. To this end, future efforts would be best spent on understanding alignments through the theory, modelling and simulations (both hydrodynamic and semi-analytic) of galaxies within their host dark matter haloes.

The theories and models of the alignments of galaxies span large and small scales for both early- and late-type galaxies, with reasonable success. However, even for samples of bright elliptical galaxies, for which detailed and high-signal-to-noise measurements exist, the intermediate-scales between $1-10 h^{-1} \mathrm{Mpc}$ are still proving difficult to model accurately. Work is ongoing in this area and it is expected that a model that accounts for clustering, halo bias and redshift-space distortions will effectively unify the small, intermediate, and large scales. In the case of disc galaxies, observations generally yield marginal detections at best, so that only future data will be able to tell if the prevailing models based on tidal torque theory provide good descriptions of a variety of alignment processes.

Simulations also provide much needed insight into galaxy intrinsic alignments on all scales. Only with suites of $\mathrm{N}$-body and hydrodynamic simulations will it be possible to quantify alignment correlations in the non-linear regime, investigate the link between the morphology of bright and dark matter, and establish the physical mechanisms behind galaxy alignments - whether these are driven by the well-understood tidal stretching and torquing paradigms, or by 
purely non-linear causes such as vorticity effects. It is, however, important to be realistic about the limitations of simulations. For example, the sub-grid physics in hydrodynamic simulations are still poorly understood and represent a large area of uncertainty in the simulations. Additionally, reproducing realistic ellipticity distributions in hydrodynamic simulations may never be possible due to resolution effects. This is not a reason to disregard simulations; it is simply a reminder to use caution when interpreting the results presented, particularly when the studies do not adhere to the resolution requirements outlined in Section 7.1.

The continued study of alignments is important for both understanding galaxy formation and evolution and for mitigating the intrinsic alignment effect in cosmological weak lensing surveys. In particular, upcoming billion dollarclass telescope missions like Euclid (Laureijs et al. 2011), the Large Synoptic Survey Telescope (LSST; LSST Science Collaboration et al. 2009) and the Wide Field InfraRed Survey Telescope (WFIRST; Spergel et al. 2015) can only reach their full potential if the effects of intrinsic alignments can be mitigated from the observations. Continued efforts into modelling the mildly non-linear scales and pursuit of the roadmap outlined here will both increase our understanding of the formation and evolution of intrinsic alignments on all scales and also provide data sets with known parameters to develop and test mitigation techniques.

\section{Acknowledgments}

We acknowledge the support of the International Space Science Institute Bern for two workshops at which this work was conceived. We would like to thank Henk Hoekstra for very useful discussions and for his comments on drafts of this work. We would also like to thank the anonymous referee for their careful reading and detailed suggestions that helped to improve the clarity of this review. We are grateful to J. Blazek, S. Singh and M. Velliscig for sharing their data and figures. We would also like to thank the participants of the Lorentz workshop: Extracting information from weak lensing: Small scales=Big problem, for their useful discussions and insights.

AK was supported in part by JPL, run under a contract by Caltech for NASA. AK was also supported in part by NASA ROSES 13-ATP13-0019 and NASA ROSES 12-EUCLID12-0004. MC was supported by the Netherlands organisation for Scientific Research (NWO) Vidi grant 639.042.814. BJ acknowledges support by an STFC Ernest Rutherford Fellowship, grant reference ST/J004421/1. TDK is supported by a Royal Society URF. AL acknowledges the support of the European Union Seventh Framework Programme (FP7/2007-2013) under grant agreement number 624151. RM acknowledges the support of NASA ROSES 12-EUCLID12-0004. CS acknowledges support from the European Research Council under FP7 grant number 279396. MLB is supported by the European Research Council (EC FP7 grant number 280127) and by a STFC Advanced/Halliday fellowship (grant number ST/I005129/1).

\section{REFERENCES}

Abadi, M. G., Navarro, J. F., Fardal, M., Babul, A., \& Steinmetz, M. 2010, Galaxy-induced transformation of dark matter haloes, MNRAS, 407, 435 [39]

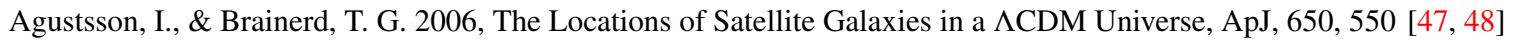

—. 2010, Anisotropic Locations of Satellite Galaxies: Clues to the Orientations of Galaxies within their Dark Matter Halos, ApJ, 709,1321 [47]

Allgood, B., Flores, R. A., Primack, J. R., et al. 2006, The shape of dark matter haloes: dependence on mass, redshift, radius and formation, MNRAS, 367, 1781 [27, 28]

Altay, G., Colberg, J. M., \& Croft, R. A. C. 2006, The influence of large-scale structures on halo shapes and alignments, MNRAS, $370,1422[33,34]$

Angulo, R. E., Lacey, C. G., Baugh, C. M., \& Frenk, C. S. 2009, The fate of substructures in cold dark matter haloes, MNRAS, 399,983 [31]

Aragón-Calvo, M. A., van de Weygaert, R., Jones, B. J. T., \& van der Hulst, J. M. 2007, Spin Alignment of Dark Matter Halos in Filaments and Walls, ApJ, 655, L5 [26, 35, 36]

Aragon-Calvo, M. A., \& Yang, L. F. 2014, The hierarchical nature of the spin alignment of dark matter haloes in filaments, MNRAS, 440, L46 [35, 36] 
Avila-Reese, V., Colín, P., Gottlöber, S., Firmani, C., \& Maulbetsch, C. 2005, The Dependence on Environment of Cold Dark Matter Halo Properties, ApJ, 634, 51 [27, 28]

Bailin, J., Power, C., Norberg, P., Zaritsky, D., \& Gibson, B. K. 2008, The anisotropic distribution of satellite galaxies, MNRAS, 390, 1133 [47]

Bailin, J., \& Steinmetz, M. 2005, Internal and External Alignment of the Shapes and Angular Momenta of $\Lambda$ CDM Halos, ApJ, 627, 647 [26, 27, 28, 30, 33, 34]

Bailin, J., Kawata, D., Gibson, B. K., et al. 2005, Internal Alignment of the Halos of Disk Galaxies in Cosmological Hydrodynamic Simulations, ApJ, 627, L17 [27, 39, 46]

Barnes, J., \& Efstathiou, G. 1987, Angular momentum from tidal torques, ApJ, 319, 575 [28, 33, 34]

Bartelmann, M., \& Schneider, P. 2001, Weak gravitational lensing, Phys. Rep., 340, 291 [3]

Berentzen, I., \& Shlosman, I. 2006, Growing Live Disks within Cosmologically Assembling Asymmetric Halos: Washing Out the Halo Prolateness, ApJ, 648, 807 [39]

Berlind, A. A., \& Weinberg, D. H. 2002, The Halo Occupation Distribution: Toward an Empirical Determination of the Relation between Galaxies and Mass, ApJ, 575, 587 [48]

Bernardeau, F., Bonvin, C., \& Vernizzi, F. 2010, Full-sky lensing shear at second order, Phys. Rev. D, 81, 083002 [9]

Bernstein, G. M., \& Jarvis, M. 2002, Shapes and Shears, Stars and Smears: Optimal Measurements for Weak Lensing, AJ, 123, 583 [4]

Bett, P. 2012, Halo shapes from weak lensing: the impact of galaxy-halo misalignment, MNRAS, 420, 3303 [48]

Bett, P., Eke, V., Frenk, C. S., et al. 2007, The spin and shape of dark matter haloes in the Millennium simulation of a $\Lambda$ cold dark matter universe, MNRAS, 376, 215 [23, 24, 27, 28, 30, 49, 50]

Bett, P., Eke, V., Frenk, C. S., Jenkins, A., \& Okamoto, T. 2010, The angular momentum of cold dark matter haloes with and without baryons, MNRAS, 404, 1137 [39]

Bett, P. E., \& Frenk, C. S. 2012, Spin flips - I. Evolution of the angular momentum orientation of Milky Way-mass dark matter haloes, MNRAS, 420, 3324 [30]

Blazek, J., Mandelbaum, R., Seljak, U., \& Nakajima, R. 2012, Separating intrinsic alignment and galaxy-galaxy lensing, J. Cosmology Astropart. Phys., 5, 41 [13, 18]

Blazek, J., McQuinn, M., \& Seljak, U. 2011, Testing the tidal alignment model of galaxy intrinsic alignment, J. Cosmology Astropart. Phys., 5, 10 [18]

Blazek, J., Vlah, Z., \& Seljak, U. 2015, Tidal alignment of galaxies, J. Cosmology Astropart. Phys., 8, 015 [18, 19]

Bond, J. R., Cole, S., Efstathiou, G., \& Kaiser, N. 1991, Excursion set mass functions for hierarchical Gaussian fluctuations, ApJ, $379,440[17,31]$

Bower, R. G., Benson, A. J., Malbon, R., et al. 2006, Breaking the hierarchy of galaxy formation, MNRAS, 370,645 [47, 48]

Boylan-Kolchin, M., Springel, V., White, S. D. M., Jenkins, A., \& Lemson, G. 2009, Resolving cosmic structure formation with the Millennium-II Simulation, MNRAS, 398, 1150 [28]

Bridle, S., \& King, L. 2007, Dark energy constraints from cosmic shear power spectra: impact of intrinsic alignments on photometric redshift requirements, New Journal of Physics, 9, 444 [12, 18]

Brown, M. L., Taylor, A. N., Hambly, N. C., \& Dye, S. 2002, Measurement of intrinsic alignments in galaxy ellipticities, MNRAS, 333, 501 [12]

Brunino, R., Trujillo, I., Pearce, F. R., \& Thomas, P. A. 2007, The orientation of galaxy dark matter haloes around cosmic voids, MNRAS, 375, 184 [35]

Bryan, S. E., Kay, S. T., Duffy, A. R., et al. 2013, The impact of baryons on the spins and shapes of dark matter haloes, MNRAS, 429, 3316 [42]

Bullock, J. S., Dekel, A., Kolatt, T. S., et al. 2001, A Universal Angular Momentum Profile for Galactic Halos, ApJ, 555, 240 [25, $27,28,30]$

Camelio, G., \& Lombardi, M. 2015, On the origin of intrinsic alignment in cosmic shear measurements: an analytic argument, A\&A, 575, A113 [13] 
Capranico, F., Merkel, P. M., \& Schäfer, B. M. 2013, Intrinsic ellipticity correlations of galaxies: models, likelihoods and interplay with weak lensing, MNRAS, 435, 194 [16]

Catelan, P., Kamionkowski, M., \& Blandford, R. D. 2001, Intrinsic and extrinsic galaxy alignment, MNRAS, 320, L7 [12, 14]

Catelan, P., \& Theuns, T. 1996, Evolution of the angular momentum of protogalaxies from tidal torques: Zel'dovich approximation, MNRAS, 282, 436 [14]

Cen, R. 2014, Frequent Spin Reorientation of Galaxies due to Local Interactions, ApJ, 785, L15 [20, 40, 41]

Chen, D. N., Jing, Y. P., \& Yoshikaw, K. 2003, Angular Momentum Distribution of Hot Gas and Implications for Disk Galaxy Formation, ApJ, 597, 35 [40]

Chisari, N. E., Mandelbaum, R., Strauss, M. A., Huff, E. M., \& Bahcall, N. A. 2014, Intrinsic alignments of group and cluster galaxies in photometric surveys, MNRAS, 445, 726 [18]

Codis, S., Pichon, C., Devriendt, J., et al. 2012, Connecting the cosmic web to the spin of dark haloes: implications for galaxy formation, MNRAS, 427, 3320 [25, 35, 36, 42]

Codis, S., Pichon, C., \& Pogosyan, D. 2015a, Spin alignments within the cosmic web: a theory of constrained tidal torques near filaments, MNRAS, 452, 3369 [14]

Codis, S., Gavazzi, R., Dubois, Y., et al. 2015b, Intrinsic alignment of simulated galaxies in the cosmic web: implications for weak lensing surveys, MNRAS, 448, 3391 [42, 43]

Cole, S., \& Lacey, C. 1996, The structure of dark matter haloes in hierarchical clustering models, MNRAS, 281, 716 [27]

Cooray, A., \& Hu, W. 2002, Second-Order Corrections to Weak Lensing by Large-Scale Structure, ApJ, 574, 19 [9]

Cooray, A., \& Milosavljević, M. 2005, What is $\mathrm{L}_{*}$ ? Anatomy of the Galaxy Luminosity Function, ApJ, 627, L89 [48]

Cooray, A., \& Sheth, R. 2002, Halo models of large scale structure, Phys. Rep., 372, 1 [16]

Coupon, J., Arnouts, S., van Waerbeke, L., et al. 2015, The galaxy-halo connection from a joint lensing, clustering and abundance analysis in the CFHTLenS/VIPERS field, MNRAS, 449, 1352 [3]

Crain, R. A., Schaye, J., Bower, R. G., et al. 2015, The EAGLE simulations of galaxy formation: calibration of subgrid physics and model variations, MNRAS, 450, 1937 [45]

Crittenden, R. G., Natarajan, P., Pen, U.-L., \& Theuns, T. 2001, Spin-induced Galaxy Alignments and Their Implications for Weak-Lensing Measurements, ApJ, 559, 552 [14, 15, 16]

—. 2002, Discriminating Weak Lensing from Intrinsic Spin Correlations Using the Curl-Gradient Decomposition, ApJ, 568, 20 [16]

Croft, R. A. C., \& Metzler, C. A. 2000, Weak-Lensing Surveys and the Intrinsic Correlation of Galaxy Ellipticities, ApJ, 545, 561 $[27,33,34]$

Cuesta, A. J., Betancort-Rijo, J. E., Gottlöber, S., et al. 2008, Spin alignment of dark matter haloes in the shells of the largest voids, MNRAS, 385, 867 [35, 36]

Danovich, M., Dekel, A., Hahn, O., Ceverino, D., \& Primack, J. 2015, Four phases of angular-momentum buildup in high-z galaxies: from cosmic-web streams through an extended ring to disc and bulge, MNRAS, 449, 2087 [20]

Davis, A. J., \& Natarajan, P. 2009, Angular momentum and clustering properties of early dark matter haloes, MNRAS, 393,1498 [27]

Davis, M., Efstathiou, G., Frenk, C. S., \& White, S. D. M. 1985, The evolution of large-scale structure in a universe dominated by cold dark matter, ApJ, 292, 371 [22, 27]

Deason, A. J., McCarthy, I. G., Font, A. S., et al. 2011, Mismatch and misalignment: dark haloes and satellites of disc galaxies, MNRAS, 415, 2607 [39, 41, 46]

Debattista, V. P., Roškar, R., Valluri, M., et al. 2013, What's up in the Milky Way? The orientation of the disc relative to the triaxial halo, MNRAS, 434, 2971 [40]

Debattista, V. P., van den Bosch, F. C., Roškar, R., et al. 2015, Internal alignments of red versus blue discs in dark matter haloes, MNRAS, 452, 4094 [40]

Dekel, A., Birnboim, Y., Engel, G., et al. 2009, Cold streams in early massive hot haloes as the main mode of galaxy formation, Nature, 457, 451 [20] 
Dodelson, S. 2003, Modern cosmology [3]

Dong, X. C., Lin, W. P., Kang, X., et al. 2014, The Distribution of Satellites around Central Galaxies in a Cosmological Hydrodynamical Simulation, ApJ, 791, L33 [46]

Dubinski, J., \& Carlberg, R. G. 1991, The structure of cold dark matter halos, ApJ, 378, 496 [28]

Dubois, Y., Pichon, C., Welker, C., et al. 2014, Dancing in the dark: galactic properties trace spin swings along the cosmic web, MNRAS, 444, 1453 [20, 42, 43]

Dutton, A. A., \& Macciò, A. V. 2014, Cold dark matter haloes in the Planck era: evolution of structural parameters for Einasto and NFW profiles, MNRAS, 441, 3359 [16]

Faltenbacher, A., Gottlöber, S., Kerscher, M., \& Müller, V. 2002, Correlations in the orientations of galaxy clusters, A\&A, 395, 1 $[26,34]$

Faltenbacher, A., Jing, Y. P., Li, C., et al. 2008, Spatial and Kinematic Alignments between Central and Satellite Halos, ApJ, 675, $146[31,32]$

Faltenbacher, A., Li, C., White, S. D. M., et al. 2009, Alignment between galaxies and large-scale structure, Research in Astronomy and Astrophysics, 9, 41 [28, 47]

Fedeli, C. 2014, The clustering of baryonic matter. I: a halo-model approach, J. Cosmology Astropart. Phys., 4, 28 [16]

Fedeli, C., Semboloni, E., Velliscig, M., et al. 2014, The clustering of baryonic matter. II: halo model and hydrodynamic simulations, J. Cosmology Astropart. Phys., 8, 28 [16]

Forero-Romero, J. E., Contreras, S., \& Padilla, N. 2014, Cosmic web alignments with the shape, angular momentum and peculiar velocities of dark matter haloes, MNRAS, 443, 1090 [25, 35, 36, 37]

Frenk, C. S., White, S. D. M., Davis, M., \& Efstathiou, G. 1988, The formation of dark halos in a universe dominated by cold dark matter, ApJ, 327, 507 [27, 28]

Gao, L., \& White, S. D. M. 2007, Assembly bias in the clustering of dark matter haloes, MNRAS, 377, L5 [16]

Giahi, A., \& Schäfer, B. M. 2013, Evolution of intrinsic ellipticity correlations due to peculiar motion, MNRAS, 428, 1312 [20]

Giahi-Saravani, A., \& Schäfer, B. M. 2014, Weak gravitational lensing of intrinsically aligned galaxies, MNRAS, 437, 1847 [20]

Giocoli, C., Bartelmann, M., Sheth, R. K., \& Cacciato, M. 2010, Halo model description of the non-linear dark matter power spectrum at $\mathrm{k} \gg 1 \mathrm{Mpc}^{-1}$, MNRAS, 408, 300 [16]

Gustafsson, M., Fairbairn, M., \& Sommer-Larsen, J. 2006, Baryonic pinching of galactic dark matter halos, Phys. Rev. D, 74, 123522 [39]

Hahn, O., Porciani, C., Carollo, C. M., \& Dekel, A. 2007a, Properties of dark matter haloes in clusters, filaments, sheets and voids, MNRAS, 375, 489 [25, 27, 34, 35, 36]

Hahn, O., Carollo, C. M., Porciani, C., \& Dekel, A. 2007b, The evolution of dark matter halo properties in clusters, filaments, sheets and voids, MNRAS, 381, 41 [25, 27, 35, 36]

Hahn, O., Teyssier, R., \& Carollo, C. M. 2010, The large-scale orientations of disc galaxies, MNRAS, 405, 274 [20, 40, 42]

Hall, A., \& Taylor, A. 2014, Intrinsic alignments in the cross-correlation of cosmic shear and cosmic microwave background weak lensing, MNRAS, 443, L119 [18]

Hatton, S., \& Ninin, S. 2001, Angular momentum alignment of dark matter haloes, MNRAS, 322, 576 [34, 35]

Heavens, A., \& Peacock, J. 1988, Tidal torques and local density maxima, MNRAS, 232, 339 [39]

Heavens, A., Refregier, A., \& Heymans, C. 2000, Intrinsic correlation of galaxy shapes: implications for weak lensing measurements, MNRAS, 319, 649 [27, 33, 34, 48]

Hernquist, L., \& Katz, N. 1989, TREESPH - A unification of SPH with the hierarchical tree method, ApJS, 70,419 [23]

Heymans, C., Brown, M., Heavens, A., et al. 2004, Weak lensing with COMBO-17: estimation and removal of intrinsic alignments, MNRAS, 347, 895 [18, 45, 48]

Heymans, C., White, M., Heavens, A., Vale, C., \& van Waerbeke, L. 2006, Potential sources of contamination to weak lensing measurements: constraints from N-body simulations, MNRAS, 371, 750 [35, 36, 48]

Heymans, C., Grocutt, E., Heavens, A., et al. 2013, CFHTLenS tomographic weak lensing cosmological parameter constraints: Mitigating the impact of intrinsic galaxy alignments, MNRAS, 432, $2433[13,18]$ 
Hirata, C. M., \& Seljak, U. 2004, Intrinsic alignment-lensing interference as a contaminant of cosmic shear, Phys. Rev. D, 70, $063526[12,13,15]$

—. 2010, Intrinsic alignment-lensing interference as a contaminant of cosmic shear, ArXiv Astrophysics e-prints, arXiv:astro$\mathrm{ph} / 0406275$ [12]

Hirata, C. M., Mandelbaum, R., Seljak, U., et al. 2004, Galaxy-galaxy weak lensing in the Sloan Digital Sky Survey: intrinsic alignments and shear calibration errors, MNRAS, 353, 529 [13]

Hockney, R. W., \& Eastwood, J. W. 1981, Computer Simulation Using Particles [42]

Hoekstra, H., Yee, H. K. C., \& Gladders, M. D. 2004, Properties of Galaxy Dark Matter Halos from Weak Lensing, ApJ, 606, 67 [3]

Hopkins, P. F., Bahcall, N. A., \& Bode, P. 2005, Cluster Alignments and Ellipticities in ACDM Cosmology, ApJ, 618, 1 [26, 27, $33,34]$

Jing, Y. P. 2002, Intrinsic correlation of halo ellipticity and its implications for large-scale weak lensing surveys, MNRAS, 335, L89 [23, 27, 33, 34]

Jing, Y. P., \& Suto, Y. 2002, Triaxial Modeling of Halo Density Profiles with High-Resolution N-Body Simulations, ApJ, 574,538 $[23,28,31]$

Joachimi, B., Mandelbaum, R., Abdalla, F. B., \& Bridle, S. L. 2011, Constraints on intrinsic alignment contamination of weak lensing surveys using the MegaZ-LRG sample, A\&A, 527, A26 [12, 48, 49, 50]

Joachimi, B., Semboloni, E., Bett, P. E., et al. 2013a, Intrinsic galaxy shapes and alignments - I. Measuring and modelling COSMOS intrinsic galaxy ellipticities, MNRAS, 431, 477 [48, 49]

Joachimi, B., Semboloni, E., Hilbert, S., et al. 2013b, Intrinsic galaxy shapes and alignments - II. Modelling the intrinsic alignment contamination of weak lensing surveys, MNRAS, 436, 819 [48, 49]

Joachimi, B., Cacciato, M., Kitching, T. D., et al. 2015, Galaxy Alignments: An Overview, Space Sci. Rev., 193, 1 [4, 9, 12, 51]

Kaiser, N. 1987, Clustering in real space and in redshift space, MNRAS, 227, 1 [9]

Kamionkowski, M., Kosowsky, A., \& Stebbins, A. 1997, Statistics of cosmic microwave background polarization, Phys. Rev. D, $55,7368[8]$

Kang, X., Mao, S., Gao, L., \& Jing, Y. P. 2005, Are great disks defined by satellite galaxies in Milky-Way type halos rare in $\Lambda$ CDM?, A\&A, 437, 383 [47]

Kang, X., van den Bosch, F. C., Yang, X., et al. 2007, The alignment between satellites and central galaxies: theory versus observations, MNRAS, 378, $1531[47,48]$

Kasun, S. F., \& Evrard, A. E. 2005, Shapes and Alignments of Galaxy Cluster Halos, ApJ, 629, 781 [26, 27, 34]

Kazantzidis, S., Kravtsov, A. V., Zentner, A. R., et al. 2004, The Effect of Gas Cooling on the Shapes of Dark Matter Halos, ApJ, 611, L73 [39]

Khandai, N., Di Matteo, T., Croft, R., et al. 2015, The MassiveBlack-II simulation: the evolution of haloes and galaxies to z 0, MNRAS, 450, 1349 [38, 45]

Kirk, D., Laszlo, I., Bridle, S., \& Bean, R. 2013, Optimizing cosmic shear surveys to measure modifications to gravity on cosmic scales, MNRAS, 430, 197 [18]

Kirk, D., Brown, M. L., Hoekstra, H., et al. 2015, Galaxy Alignments: Observations and Impact on Cosmology, Space Sci. Rev., 193, $139[3,4,13,21,22,43,45,47,48]$

Klypin, A., \& Holtzman, J. 1997, Particle-Mesh code for cosmological simulations, ArXiv Astrophysics e-prints, astro-ph/9712217 [22]

Knebe, A., Draganova, N., Power, C., et al. 2008a, On the relation between the radial alignment of dark matter subhaloes and host mass in cosmological simulations, MNRAS, 386, L52 [31, 48]

Knebe, A., Gill, S. P. D., Gibson, B. K., et al. 2004, Anisotropy in the Distribution of Satellite Galaxy Orbits, ApJ, 603,7 [31]

Knebe, A., Libeskind, N. I., Knollmann, S. R., et al. 2010, The impact of baryonic physics on the shape and radial alignment of substructures in cosmological dark matter haloes, MNRAS, 405, 1119 [39, 41]

Knebe, A., Yahagi, H., Kase, H., Lewis, G., \& Gibson, B. K. 2008b, The radial alignment of dark matter subhaloes: from simulations to observations, MNRAS, 388, L34 [31, 33] 
Knebe, A., Knollmann, S. R., Muldrew, S. I., et al. 2011, Haloes gone MAD: The Halo-Finder Comparison Project, MNRAS, 415, $2293[23]$

Krause, E., \& Hirata, C. M. 2010, Weak lensing power spectra for precision cosmology. Multiple-deflection, reduced shear, and lensing bias corrections, A\&A, 523, A28 [9]

Kuhlen, M., Diemand, J., \& Madau, P. 2007, The Shapes, Orientation, and Alignment of Galactic Dark Matter Subhalos, ApJ, 671, $1135[24,28,31,33]$

Lacey, C., \& Cole, S. 1994, Merger Rates in Hierarchical Models of Galaxy Formation - Part Two - Comparison with N-Body Simulations, MNRAS, 271, 676 [22]

Laureijs, R., Amiaux, J., Arduini, S., et al. 2011, Euclid Definition Study Report, ArXiv e-prints, arXiv:1110.3193 [53]

Le Brun, A. M. C., McCarthy, I. G., Schaye, J., \& Ponman, T. J. 2014, Towards a realistic population of simulated galaxy groups and clusters, MNRAS, 441, 1270 [45]

Lee, J. 2011, On the Intrinsic Alignments of the Late-type Spiral Galaxies from the Sloan Digital Sky Survey Data Release 7, ApJ, 732,99 [43]

Lee, J., \& Erdogdu, P. 2007, The Alignments of the Galaxy Spins with the Real-Space Tidal Field Reconstructed from the 2MASS Redshift Survey, ApJ, 671, 1248 [43]

Lee, J., Kang, X., \& Jing, Y. P. 2005, The Intrinsic Alignment of Dark Halo Substructures, ApJ, 629, L5 [34]

Lee, J., \& Pen, U.-L. 2000, Cosmic Shear from Galaxy Spins, ApJ, 532, L5 [14]

Libeskind, N. I., Cole, S., Frenk, C. S., Okamoto, T., \& Jenkins, A. 2007, Satellite systems around galaxies in hydrodynamic simulations, MNRAS, 374, 16 [41]

Libeskind, N. I., Frenk, C. S., Cole, S., Jenkins, A., \& Helly, J. C. 2009, How common is the Milky Way-satellite system alignment?, MNRAS, 399, 550 [48]

Libeskind, N. I., Hoffman, Y., Forero-Romero, J., et al. 2013, The velocity shear tensor: tracer of halo alignment, MNRAS, 428, $2489[25,35,36,37]$

Libeskind, N. I., Hoffman, Y., Knebe, A., et al. 2012, The cosmic web and the orientation of angular momenta, MNRAS, 421 , L137 [25]

LSST Science Collaboration, Abell, P. A., Allison, J., et al. 2009, LSST Science Book, Version 2.0, ArXiv e-prints, arXiv:0912.0201 [53]

MacCrann, N., Zuntz, J., Bridle, S., Jain, B., \& Becker, M. R. 2015, Cosmic discordance: are Planck CMB and CFHTLenS weak lensing measurements out of tune?, MNRAS, 451, 2877 [13]

Maller, A. H., \& Dekel, A. 2002, Towards a resolution of the galactic spin crisis: mergers, feedback and spin segregation, MNRAS, 335,487 [40]

Maller, A. H., Dekel, A., \& Somerville, R. 2002, Modelling angular-momentum history in dark-matter haloes, MNRAS, 329,423 $[27,30]$

Mandelbaum, R., Hirata, C. M., Broderick, T., Seljak, U., \& Brinkmann, J. 2006a, Ellipticity of dark matter haloes with galaxygalaxy weak lensing, MNRAS, 370, 1008 [3]

Mandelbaum, R., Hirata, C. M., Ishak, M., Seljak, U., \& Brinkmann, J. 2006b, Detection of large-scale intrinsic ellipticity-density correlation from the Sloan Digital Sky Survey and implications for weak lensing surveys, MNRAS, 367, 611 [13, 18, 48]

Mandelbaum, R., Blake, C., Bridle, S., et al. 2011, The WiggleZ Dark Energy Survey: direct constraints on blue galaxy intrinsic alignments at intermediate redshifts, MNRAS, 410, $844[18,48]$

McCarthy, I. G., Le Brun, A. M. C., Schaye, J., \& Holder, G. P. 2014, The thermal Sunyaev-Zel'dovich effect power spectrum in light of Planck, MNRAS, 440, 3645 [45]

Merkel, P. M., \& Schäfer, B. M. 2013, Intrinsic alignments and 3d weak gravitational lensing, MNRAS, 434, 1808 [16]

Metz, M., Kroupa, P., \& Libeskind, N. I. 2008, The Orbital Poles of Milky Way Satellite Galaxies: A Rotationally Supported Disk of Satellites, ApJ, 680, 287 [47]

Miralda-Escude, J. 1991, The correlation function of galaxy ellipticities produced by gravitational lensing, ApJ, 380, 1 [26]

Mo, H., van den Bosch, F. C., \& White, S. 2010, Galaxy Formation and Evolution [3] 
Monaghan, J. J., \& Lattanzio, J. C. 1985, A refined particle method for astrophysical problems, A\&A, 149, 135 [23]

Natarajan, P., \& Refregier, A. 2000, Two-Dimensional Galaxy-Galaxy Lensing: A Direct Measure of the Flattening and Alignment of Light and Mass in Galaxies, ApJ, 538, L113 [3]

Navarro, J. F., Abadi, M. G., \& Steinmetz, M. 2004, Tidal Torques and the Orientation of Nearby Disk Galaxies, ApJ, 613, L41 [42]

Navarro, J. F., \& Benz, W. 1991, Dynamics of cooling gas in galactic dark halos, ApJ, 380, 320 [40]

Navarro, J. F., Frenk, C. S., \& White, S. D. M. 1996, The Structure of Cold Dark Matter Halos, ApJ, 462, 563 [19]

—. 1997, A Universal Density Profile from Hierarchical Clustering, ApJ, 490, 493 [16, 17]

Ocean Wang, Y., Lin, W. P., Kang, X., et al. 2014, Satellite Alignment. I. Distribution of Substructures and their Dependence on Assembly History from N-body Simulations, ApJ, 786, 8 [31]

Okamoto, T., Eke, V. R., Frenk, C. S., \& Jenkins, A. 2005, Effects of feedback on the morphology of galaxy discs, MNRAS, 363, 1299 [30]

Okumura, T., \& Jing, Y. P. 2009, The Gravitational Shear-Intrinsic Ellipticity Correlation Functions of Luminous Red Galaxies in Observation and in the $\Lambda$ CDM Model, ApJ, 694, L83 [47]

Okumura, T., Jing, Y. P., \& Li, C. 2009, Intrinsic Ellipticity Correlation of SDSS Luminous Red Galaxies and Misalignment with Their Host Dark Matter Halos, ApJ, 694, 214 [13, 45, 46, 47]

Onuora, L. I., \& Thomas, P. A. 2000, The alignment of clusters using large-scale simulations, MNRAS, 319, 614 [33, 34]

Parker, L. C., Hoekstra, H., Hudson, M. J., van Waerbeke, L., \& Mellier, Y. 2007, The Masses and Shapes of Dark Matter Halos from Galaxy-Galaxy Lensing in the CFHT Legacy Survey, ApJ, 669, 21 [3]

Patiri, S. G., Cuesta, A. J., Prada, F., Betancort-Rijo, J., \& Klypin, A. 2006, The Alignment of Dark Matter Halos with the Cosmic Web, ApJ, 652, L75 [35]

Paz, D. J., Lambas, D. G., Padilla, N., \& Merchán, M. 2006, Shapes of clusters and groups of galaxies: comparison of model predictions with observations, MNRAS, 366, 1503 [27]

Paz, D. J., Sgró, M. A., Merchán, M., \& Padilla, N. 2011, Alignments of galaxy group shapes with large-scale structure, MNRAS, 414, 2029 [35]

Paz, D. J., Stasyszyn, F., \& Padilla, N. D. 2008, Angular momentum-large-scale structure alignments in $\Lambda$ CDM models and the SDSS, MNRAS, 389, 1127 [28, 36]

Peebles, P. J. E. 1969, Origin of the Angular Momentum of Galaxies, ApJ, 155, 393 [25]

Pereira, M. J., \& Bryan, G. L. 2010, Tidal Torquing of Elliptical Galaxies in Cluster Environments, ApJ, 721, 939 [32, 33]

Pereira, M. J., Bryan, G. L., \& Gill, S. P. D. 2008, Radial Alignment in Simulated Clusters, ApJ, 672, 8 25 [31, 33]

Porciani, C., Dekel, A., \& Hoffman, Y. 2002, Testing tidal-torque theory - I. Spin amplitude and direction, MNRAS, 332,325 [20, 24, 34]

Press, W. H., \& Schechter, P. 1974, Formation of Galaxies and Clusters of Galaxies by Self-Similar Gravitational Condensation, ApJ, 187, 425 [17, 21, 31]

Ragone-Figueroa, C., \& Plionis, M. 2007, Environmental influences on the morphology and dynamics of group-sized haloes, MNRAS, 377, 1785 [27, 28, 30]

Romano-Díaz, E., Shlosman, I., Heller, C., \& Hoffman, Y. 2009, Dissecting Galaxy Formation. I. Comparison Between Pure Dark Matter and Baryonic Models, ApJ, 702, 1250 [30, 40]

Scannapieco, C., White, S. D. M., Springel, V., \& Tissera, P. B. 2009, The formation and survival of discs in a $\Lambda$ CDM universe, MNRAS, 396, 696 [30, 40]

Schäfer, B. M. 2009, Galactic Angular Momenta and Angular Momentum Correlations in the Cosmological Large-Scale Structure, International Journal of Modern Physics D, 18, 173 [24]

Schäfer, B. M., \& Merkel, P. M. 2012, Galactic angular momenta and angular momentum couplings in the large-scale structure, MNRAS, 421, 2751 [14, 15]

Schaye, J., Dalla Vecchia, C., Booth, C. M., et al. 2010, The physics driving the cosmic star formation history, MNRAS, 402,1536 $[42,45]$ 
Schaye, J., Crain, R. A., Bower, R. G., et al. 2015, The EAGLE project: simulating the evolution and assembly of galaxies and their environments, MNRAS, 446, 521 [45]

Schneider, M. D., \& Bridle, S. 2010, A halo model for intrinsic alignments of galaxy ellipticities, MNRAS, 402, 2127 [17, 18, 19]

Schneider, M. D., Frenk, C. S., \& Cole, S. 2012, The shapes and alignments of dark matter halos, J. Cosmology Astropart. Phys., $5,30[27,28,29,33,34,51]$

Schrabback, T., Hilbert, S., Hoekstra, H., et al. 2015, CFHTLenS: weak lensing constraints on the ellipticity of galaxy-scale matter haloes and the galaxy-halo misalignment, MNRAS, 454, 1432 [3]

Seljak, U. 1997, Measuring Polarization in the Cosmic Microwave Background, ApJ, 482, 6 [8]

—. 2000, Analytic model for galaxy and dark matter clustering, MNRAS, 318, 203 [16]

Sharma, S., \& Steinmetz, M. 2005, The Angular Momentum Distribution of Gas and Dark Matter in Galactic Halos, ApJ, 628, 21 [40]

Shaw, L. D., Weller, J., Ostriker, J. P., \& Bode, P. 2006, Statistics of Physical Properties of Dark Matter Clusters, ApJ, 646, 815 $[27,28]$

Singh, S., Mandelbaum, R., \& More, S. 2015, Intrinsic alignments of SDSS-III BOSS LOWZ sample galaxies, MNRAS, 450,2195 $[9,18,19,51]$

Sousbie, T., Colombi, S., \& Pichon, C. 2009, The fully connected N-dimensional skeleton: probing the evolution of the cosmic web, MNRAS, 393, 457 [26]

Sousbie, T., Pichon, C., Colombi, S., Novikov, D., \& Pogosyan, D. 2008, The 3D skeleton: tracing the filamentary structure of the Universe, MNRAS, 383, 1655 [35, 36]

Spergel, D., Gehrels, N., Baltay, C., et al. 2015, Wide-Field InfrarRed Survey Telescope-Astrophysics Focused Telescope Assets WFIRST-AFTA 2015 Report, ArXiv e-prints, arXiv:1503.03757 [53]

Springel, V., White, S. D. M., Jenkins, A., et al. 2005, Simulations of the formation, evolution and clustering of galaxies and quasars, Nature, 435, 629 [28]

Tenneti, A., Mandelbaum, R., Di Matteo, T., Feng, Y., \& Khandai, N. 2014, Galaxy shapes and intrinsic alignments in the MassiveBlack-II simulation, MNRAS, 441, 470 [42, 43, 44, 45, 46, 48, 49, 50]

Tenneti, A., Singh, S., Mandelbaum, R., et al. 2015a, Intrinsic alignments of galaxies in the MassiveBlack-II simulation: analysis of two-point statistics, MNRAS, 448, 3522 [42, 45]

Tenneti, A., Mandelbaum, R., Di Matteo, T., Kiessling, A., \& Khandai, N. 2015b, Galaxy shapes and alignments in the MassiveBlack-II hydrodynamic and dark matter-only simulations, MNRAS, 453, 469 [42, 45, 46, 51]

Teyssier, R. 2002, Cosmological hydrodynamics with adaptive mesh refinement. A new high resolution code called RAMSES, A\&A, 385, 337 [42]

Tinker, J., Kravtsov, A. V., Klypin, A., et al. 2008, Toward a Halo Mass Function for Precision Cosmology: The Limits of Universality, ApJ, 688, 709 [16, 17]

Tinker, J. L., Robertson, B. E., Kravtsov, A. V., et al. 2010, The Large-scale Bias of Dark Matter Halos: Numerical Calibration and Model Tests, ApJ, 724, 878 [16, 17]

Tinker, J. L., Weinberg, D. H., Zheng, Z., \& Zehavi, I. 2005, On the Mass-to-Light Ratio of Large-Scale Structure, ApJ, 631, 41 [18]

Tormen, G. 1997, The rise and fall of satellites in galaxy clusters, MNRAS, 290, 411 [31]

Trowland, H. E., Lewis, G. F., \& Bland-Hawthorn, J. 2013, The Cosmic History of the Spin of Dark Matter Halos within the Large-scale Structure, ApJ, 762, 72 [24, 25, 26, 34, 35, 36]

Troxel, M. A., \& Ishak, M. 2014, Cross-correlation between cosmic microwave background lensing and galaxy intrinsic alignment as a contaminant to gravitational lensing cross-correlated probes of the Universe, Phys. Rev. D, 89, 063528 [18]

van den Bosch, F. C., Abel, T., Croft, R. A. C., Hernquist, L., \& White, S. D. M. 2002, The Angular Momentum of Gas in Protogalaxies. I. Implications for the Formation of Disk Galaxies, ApJ, 576, 21 [39, 48]

van den Bosch, F. C., Abel, T., \& Hernquist, L. 2003, The angular momentum of gas in protogalaxies - II. The impact of pre-heating, MNRAS, 346, 177 [40] 
van den Bosch, F. C., More, S., Cacciato, M., Mo, H., \& Yang, X. 2013, Cosmological constraints from a combination of galaxy clustering and lensing - I. Theoretical framework, MNRAS, 430, $725[16,18]$

van Uitert, E., Hoekstra, H., Schrabback, T., et al. 2012, Constraints on the shapes of galaxy dark matter haloes from weak gravitational lensing, A\&A, 545, A71 [3]

Velliscig, M., Cacciato, M., Schaye, J., et al. 2015, The alignment and shape of dark matter, stellar, and hot gas distributions in the EAGLE and cosmo-OWLS simulations, MNRAS, 453, $721[45,46]$

Vitvitska, M., Klypin, A. A., Kravtsov, A. V., et al. 2002, The Origin of Angular Momentum in Dark Matter Halos, ApJ, 581, 799 $[27,30]$

Vogelsberger, M., Genel, S., Springel, V., et al. 2014, Introducing the Illustris Project: simulating the coevolution of dark and visible matter in the Universe, MNRAS, 444, 1518 [38]

Wang, L., Weinmann, S. M., De Lucia, G., \& Yang, X. 2013, Detection of galaxy assembly bias, MNRAS, 433, 515 [16]

Warren, M. S., Quinn, P. J., Salmon, J. K., \& Zurek, W. H. 1992, Dark halos formed via dissipationless collapse. I - Shapes and alignment of angular momentum, ApJ, 399, 405 [27, 28]

Welker, C., Devriendt, J., Dubois, Y., Pichon, C., \& Peirani, S. 2014, Mergers drive spin swings along the cosmic web, MNRAS, 445, L46 [42, 43]

York, D. G., Adelman, J., Anderson, Jr., J. E., et al. 2000, The Sloan Digital Sky Survey: Technical Summary, AJ, 120, 1579 [13]

Zel'dovich, Y. B. 1970, Gravitational instability: An approximate theory for large density perturbations., A\&A, 5, 84 [11]

Zentner, A. R., Kravtsov, A. V., Gnedin, O. Y., \& Klypin, A. A. 2005, The Anisotropic Distribution of Galactic Satellites, ApJ, $629,219[47]$

Zhang, Y., Yang, X., Faltenbacher, A., et al. 2009, The Spin and Orientation of Dark Matter Halos Within Cosmic Filaments, ApJ, $706,747[26,35,36]$

This 2-column preprint was prepared with the AAS LATEX macros v5.2. 